Universidade de São Paulo

Instituto de Física de São Carlos

William Couto Corrêa de Lima

\title{
Do mecanismo de despertar do vácuo (ou de como fazer o vácuo pesado)
}

São Carlos 

William Couto Corrêa de Lima

\section{Do mecanismo de despertar do vácuo (ou de como fazer o vácuo pesado)}

Tese apresentada ao Programa de Pósgraduação em Física do Instituto de Física de São Carlos da Universidade de São Paulo para obtenção do título de Doutor em Ciências.

Área de concentração: Física Básica.

Orientador: Prof. Dr. Daniel Augusto Turolla Vanzella.

Versão corrigida

(A versão original encontra-se na unidade que aloja o Programa.)

São Carlos 
AUTORIZO A REPRODUÇÃO E DIVULGAÇÃO TOTAL OU PARCIAL DESTE TRABALHO, POR QUALQÜER MEIO CONVENCIONAL OU ELETRÔNICO PARA FINS DE ESTUDO E PESQUISA, DESDE QUE CITADA A FONTE.

Ficha catalográfica elaborada pelo Serviço de Biblioteca e Informação do IFSC, com os dados fornecidos pelo(a) autor(a)

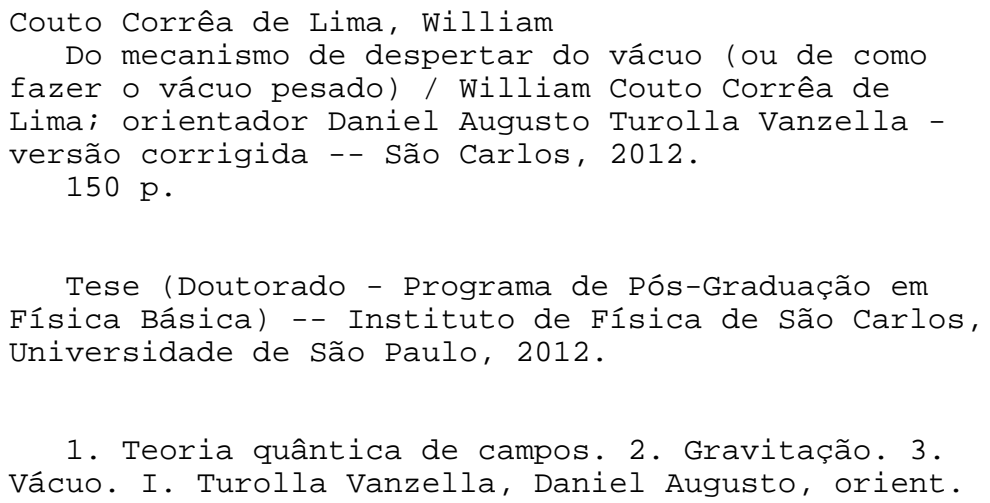

1. Teoria quântica de campos. 2. Gravitação. 3. Vácuo. I. Turolla Vanzella, Daniel Augusto, orient. II. Título. 




\section{Dedicatória}

Este estudo é dedicado à memória de meus avós paternos, Adelina Leardini de Lima e Sebastião Corrêa de Lima e de minha avó materna, Letízia Nunes de Pádua Couto. 



\section{Agradecimentos}

Há dez anos sou estudante do Instituto de Física de São Carlos, e a este Instituto e à Universidade sou enormemente grato pela vida acadêmica que me proporcionaram na última década. Por todo esse tempo que pude dedicar à minha formação acadêmica tenho muito a agradecer aos meus pais, V. L. do Couto Lima e E. Corrêa de Lima, os quais sempre tiveram especial apreço e dedicação pela formação dos filhos. Devo agradecimentos aos colegas de pós-graduação pela atmosfera agradável que ajudaram a criar em nosso ambiente de trabalho. Agradeço também ao Prof. G. E. A. Matsas por sempre nos incentivar a prosseguir com a pesquisa e por suas contribuições a este estudo. Por fim, tenho muito a agradecer ao Prof. D. A. T. Vanzella, meu guia ao longo de meus estudos de pós-graduação. Do ponto de vista acadêmico, sou-lhe grato pela atenção e cuidado que dispendeu com minha formação e com a leitura desta tese. Neste trabalho acredito ter colocado o melhor de minhas capacidades e, se isso é verdade, a ele devo o suporte para tanto. Do ponto de vista pessoal, acho que tive sorte de conhecer um ótimo indivíduo, a quem tenho como amigo. Assim, despeço-me da pós-graduação com a certeza de que uma das boas decisões que tomei nesses anos foi tornar-me seu estudante.

Este trabalho foi suportado inicialmente por uma bolsa do Conselho Nacional de Desenvolvimento Científico e Tecnológico (CNPq), processo n. 141157/2008-9, e depois por uma bolsa da Fundação de Amparo à Pesquisa do Estado de São Paulo (FAPESP), processo n. $2007 / 59830-1$.

Nota adicionada à versão corrigida: Agradeço aos membros da comissão julgadora, Prof.

S. E. C. E. Jorás, Prof. F. M. Forger, Prof. J. C. A. Barata e Prof. A. V. Saa, pelas perguntas e comentários que contribuíram para a qualidade deste texto. 

(...) quantum field theory is the quantum theory of a field, not a theory of "particles".

R. M. Wald 



\section{Resumo}

\section{LIMA, W.C.C. Do mecanismo de despertar do vácuo (ou de como fazer o vácuo}

pesado). 2012. 150 p. Tese (Doutorado) - Instituto de Física de São Carlos, Universidade de São Paulo, São Carlos, 2012.

É fato sabido que, de todas as interações fundamentais da Natureza que conhecemos, a gravitacional é a que acopla mais fracamente com a matéria. Isso sugere que na maior parte dos processos físicos a força gravitacional desempenha papel diminuto. Por outro lado, na Teoria Quântica de Campos é atribuído ao estado de vácuo uma rica estrutura, a qual é indispensável para uma descrição consistente da Natureza. No entanto, implicações experimentais diretas dessa estrutura são muito sutis e requerem sistemas cuidadosamente projetados para serem observadas, como é o caso do Efeito Casimir. À luz dos fatos mencionados acima, é de se esperar que na "fusão" minimamente consistente entre Gravitação e Mecânica Quântica, a chamada Teoria Quântica de Campos em Espaços-tempos Curvos, efeitos relacionados a perturbação do vácuo pelo campo gravitacional sejam muito difíceis de serem observados. De fato, a despeito de sua importância conceitual, o efeito de evaporação de buracos negros é praticamente impossível de ser observado para sistemas astrofísicos. No curso deste doutoramento, todavia, foi mostrado que essa crença é falsa e que é possível que existam situações em que a evolução bem comportada do espaço-tempo força a densidade de energia de vácuo a tornar-se dominante sobre a densidade de energia clássica que gera o espaço-tempo de fundo. Uma vez despertado, o vácuo passaria a dirigir a evolução do sistema gravitacional, o que poderia ter conseqüências inesperadas em contextos astrofísicos. Qualquer que seja seu destino, é razoável esperar que a retroação do vácuo aja sobre o sistema gravitacional de forma a cessar as instabilidades. Com essa simples observação é possível concluir que quando o vácuo adormece novamente processos de criação de partículas em profusão podem ser engendrados.

Palavras-chave: Teoria quântica de campos. Gravitação. Vácuo. 



\begin{abstract}
LIMA, W.C.C. On the vacuum awakening mechanism (or how to turn the vacuum into something heavy). 2012. 150 p. Tese (Doutorado) - Instituto de Física de São Carlos, Universidade de São Paulo, São Carlos, 2012.
\end{abstract}

It is well known that the gravitational interaction is the weakest among the fundamental forces in Nature. This fact suggests that Gravity plays a minor part in the majority of physical process. On the other hand, in Quantum Field Theory a rich structure is attributed to the vacuum state, which is imperative for a consistent description of the more basic processes in Nature. Nevertheless, the direct experimental implications of this structure are very subtle and their observation requires specially designed systems, as in the case of the Casimir Effect. Therefore, it is reasonable to expect that effects related to the perturbation of the quantum vacuum by gravitational fields, described by the framework of Quantum Field Theory in Curved Space-times, would be hard to be observed. This is the case of the black hole evaporation effect. In spite of its conceptual importance, this effect is virtually impossible to be observed for astrophysical black holes. Notwithstanding, here it is argued that this belief is false and that there exist well-behaved space-time evolutions where the vacuum energy density of free quantum fields is forced by the very same background space-time to become dominant over the classical energy density. Once it has been awakened, the quantum vacuum would overrule the dynamics of the entire gravitational system, which may bear some unexpected astrophysical implications. Whatever turns out to be the fate of the system, it seems reasonable to conjecture that the vacuum backreaction will act in order to cease the quantum instabilities. Through this simple observation it is possible to conclude that when the vacuum falls dormant particles are released as consequence.

Keywords: Quantum field theory. Gravitation. Vacuum. 



\section{Sumário}

1 Introdução

2 Discussões preliminares sobre geometria

2.1 Algumas definições . . . . . . . . . . . . . . . . . . . . 23

2.2 Decomposição $3+1 \ldots \ldots \ldots$. . . . . . . . . . . . . . . . . . . . . . .

3 Formalismo de quantização canônica para campos livres 35

3.1 Quantização em espaços-tempos gerais . . . . . . . . . . . . . . 36

3.2 Quantização em espaços-tempos estacionários . . . . . . . . . . . 46

3.3 Transformações de Bogoliubov . . . . . . . . . . . . . . . . . 53

3.4 O tensor energia-momentum para o campo quântico . . . . . . . . . 57

4 Um estudo da eq. de Klein-Gordon (KG) 61

4.1 Decomposição da eq. de KG . . . . . . . . . . . . . . . . . . . . . . . 61 61

4.2 Decomposição da eq. de KG em um espaço-tempo estático . . . . . . . . . 64

4.3 Decomposição da eq. de KG em um espaço-tempo estático e esfericamente simétrico . . . . . . . . . . . . . . . . . 70 
5 Do mecanismo de despertar do vácuo

5.1 Quantização do campo . . . . . . . . . . . . . . . 73

5.2 Do crescimento ilimitado das flutuações e suas implicações . . . . . . . . . 78

5.3 Uma condição necessária para o Efeito de Despertar do Vácuo . . . . . . . 80

5.4 Da energia associada ao mecanismo . . . . . . . . . . 83

6 O mecanismo em espaços-tempos particulares 85

6.1 Despertando o vácuo no interior de estrelas . . . . . . . . . . 85

6.2 Despertando o vácuo com uma casca esférica . . . . . . . . . . . . 92

7 Espaço de estados do campo na presença da instabilidade 99

7.1 Construção do espaço de estados . . . . . . . . . . . . . . . . 100

7.2 Da relação entre o efeito e barreiras de potencial parabólicas . . . . . . . . 107

8 Da criação de partículas

8.1 Criação de partículas em espaços-tempos curvos . . . . . . . . . . . . 114

8.2 Excitação de detectores . . . . . . . . . . . . . . . . . . . 116

8.3 Criação de partículas quando o vácuo adormece . . . . . . . . . . . . . . 120

9 Questões pertinentes 131

Referências 141

Apêndice A Prova da desigualdade Eq. (7.1.6) para a Eq. (7.1.5) 149 


\section{Capítulo 1}

\section{Introdução}

o longo do século XX a linguagem da Teoria Quântica de Campos mostrou-se
muito profícua para a descrição com grande acurácia dos fenômenos naturais mais fundamentais de que temos notícia. No que concerne ao problema tratado nesta tese, são de interesse aqueles fenômenos para os quais é indispensável o papel da interação gravitacional.

Atualmente, a melhor descrição de que dispomos para a Gravitação é dada pela Relatividade Geral (RG) (1-4). A RG provou-se útil para a descrição de fenômenos astrofísicos, particularmente aqueles em que há a presença de campos gravitacionais intensos, e fundamental para o entendimento da evolução do Cosmos. A RG, entretanto, não se restringe a uma teoria para a Gravitação. Ela também consiste no entendimento mais profundo que possuímos sobre a natureza do espaço e do tempo.

A formulação da Teoria Quântica de Campos na presença de campos gravitacionais como descritos pela RG é conhecida como Teoria Quântica de Campos em Espaços-tempos Curvos (TQCEC) (5 9). Esse formalismo consiste na extensão matematicamente bemposta dos conceitos mais fundamentais da Teoria Quântica de Campos em espaço plano (10 13) para geometrias mais gerais. Nos últimos 40 anos a TQCEC tem aperfeiçoado nosso entendimento sobre campos quânticos e previsto fenômenos para o quais o papel da Gravitação é determinante e que por vezes acabam por desenhar um cenário diferente 
daquele esperado pela RG.1

Talvez um dos conceitos mais surpreendentes que emerge da quantização de campos seja o chamado estado de vácuo, usualmente associado ao estado de menor energia do sistema $\left.\right|^{2}$ No contexto da Física Clássica não há nenhum princípio que impeça a remoção de todas as formas de energia de uma dada região do espaço. O conceito de vácuo cunhado pela Física até o início do século XX é o de uma região completamente vazia de energia. Já no contexto da Teoria Quântica de Campos a imagem é drasticamente diferente. É por princípio impossível remover toda a energia de uma dada região; a energia ali flutua em torno de algum valor médio que não é necessariamente nulo. Sob o olhar da Física Moderna, então, o vazio tem uma estrutura intrínseca a qual subjaz todo o mundo a nossa volta. Para campos livres no espaço-tempo de Minkowski essas flutuações quânticas da energia do vácuo são usualmente fraseadas como uma constante criação e aniquilação de pares virtuais de quanta do campo. Esses quanta são ditos virtuais pois não existem por tempo suficiente para serem detectados por observadores clássicos.

Apesar de um tanto evasiva, há casos em que é possível testar as conseqüências da estrutura do vácuo. Certamente o mais emblemático é o Efeito Casimir, onde a presença de bordas no espaço-tempo de Minkowski perturba o vácuo e tal perturbação se traduz numa força sobre as bordas (16). Apesar de surpreendente, esse fenômeno é muito sutil e requer sistemas cuidadosamente projetados para ser observado (17,18). Uma vez que das quatro forças fundamentais a gravitacional é aquela que acopla mais fracamente com a matéria, pode-se presumir que as conseqüências da perturbação do vácuo de um sistema quântico por um campo gravitacional serão ainda mais sutis. De fato, essa não é uma crença infundada haja visto que, e.g., na taxa atual a expansão do Universo é capaz de produzir menos de um méson $\pi$ por segundo no volume compreendido pelo Universo observável (19) e que no caso da radiação térmica emitida por buracos negros, o chamado

\footnotetext{
${ }^{1}$ Para uma revisão sobre a história e o presente status da TQCEC veja as referências $(14,15)$.

${ }^{2}$ Uma vez que o conceito global de energia que é conservada pela evolução só é possível quando há uma isometria temporal, em espaços-tempos gerais nem sempre é possível associar um estado do campo ao de menor energia do sistema. Entretanto, como será visto no Capítulo 3 , isso não implica em dificuldades para a definição de "estados de vácuo" - embora possa haver dificuldade em se selecionar um destes —, haja visto que na formulação canônica da TQCEC tal definição é independente da existência de isometrias temporais.
} 
Efeito Hawking, a temperatura das partículas que chegam ao infinito é da ordem de $10^{-6} \mathrm{~K}$ para sistemas gravitacionais com massa da ordem da do Sol $\left(\sim 10^{33} \mathrm{~g}\right)(20)$. Uma vez que a temperatura é inversamente proporcional à massa do sistema, o Efeito Hawking é praticamente impossível de ser observado em sistemas astrofísicos.

Existem, no entanto, situações que contrariam a crença elaborada acima. Esse é o caso do fenômeno motivo desta tese. Os resultados obtidos mostram que no contexto da TQCEC é possível existir situações nas quais a evolução bem-comportada do espaçotempo força a densidade de energia do vácuo de um campo livre a se tornar dominante sobre a densidade de energia clássica responsável pela curvatura daquele espaço-tempo de fundo. Isso se dá devido ao crescimento exponencial experimentado durante uma certa janela de tempo pelo valor esperado no vácuo de observáveis relativos ao campo. Desse modo, um sistema outrora perfeitamente estável desenvolve uma instabilidade por força da evolução do campo gravitacional ao qual está sujeito. Uma vez comparável à densidade de energia da matéria clássica, é imperativo levar em conta o vácuo como fonte de campo gravitacional no lado direito da equação de Einstein. Somente levando em conta a retroação do campo quântico sobre o espaço-tempo de fundo é possível determinar quais as conseqüências do efeito sobre o sistema físico. Essa última parte é uma questão bastante complicada, já que requer o cálculo autoconsistente do campo gravitacional, via a equação de Einstein semiclássica, e das flutuações do campo quântico. Apesar dessa dificuldade, é possível estimar a escala de tempo para que a retroação do campo quântico seja inelutável. Para corpos compactos, como estrelas de nêutrons, essa escala é da ordem de frações de milésimos de segundo, enquanto que em contextos cosmológicos seria da ordem de bilhões de anos se o efeito for desencadeado.

Além de nos debruçarmos sobre as questões relativas ao crescimento sem-limites das flutuações do vácuo, também exploramos, em tempos mais recentes, a relação entre esse crescimento e fenômenos de produção de partículas. Essa não é uma relação óbvia, haja visto que mesmo uma evolução arbitrariamente lenta do espaço-tempo seria capaz de desencadear o crescimento exponencial das flutuações do campo quântico. A despeito disso, é possível mostrar que detectores de partículas excitam copiosamente durante a fase de 
instabilidade, mesmo não sendo possível associar essas excitações ao conteúdo de partícula do sistema. É razoável supor, ainda, que essa instabilidade não deva se estender por um período arbitrariamente longo; o processo de retroação do campo quântico sobre o espaçotempo deve ser capaz de levar o sistema de volta a algum estado estacionário. Mesmo sem levar em conta o campo quântico como fonte de campo gravitacional, mostramos que esse crescimento exponencial deve dar alguma contribuição ao conteúdo de partícula do sistema numa fase estacionária ulterior à presença da instabilidade.

A idéia que originou este trabalho de pesquisa nasceu das análises realizadas por G. E. A. Matsas, do Instituto de Física Teórica da Universidade Estadual Paulista "Júlio de Mesquita Filho", enquanto preparava um trabalho em que ele e D. A. T. Vanzella são co-autores e que foi publicado no ano de 2002 (21). Foi Matsas o primeiro a notar que na região interior de estrelas suficientemente densas o potencial efetivo que comparece na equação de Klein-Gordon (KG) pode torna-se negativo. Segundo o próprio Matsas, à época ele especulava que esse potencial efetivo negativo poderia dar origem a um novo efeito de criação de partículas em espaços-tempos curvos. Imagino que a justificativa para isso fosse uma analogia com o Paradoxo de Klein (22) ou com o Efeito Schwinger, no qual partículas maciças carregadas são criadas a partir do vácuo quando o sistema está sujeito a um campo elétrico externo suficientemente intenso (23). Tanto quanto tenha chegado a mim, essa questão foi discutida com Vanzella, que naquele momento encontrava-se no curso de seu pós-doutoramento na Universidade de Wisconsin, em Milwaukee, sob a supervisão de L. Parker. Entretanto, por conta das ocupações de ambos no momento e da necessidade de darem cabo de projetos mais prementes, a questão foi engavetada. Tempos depois, já no início de 2008, eu tinha acabado de defender meu mestrado, sob orientação de Vanzella, e ingressara no doutorado procurando aprofundar meus conhecimentos em TQCEC. Inicialmente o interesse de Vanzella era analisar os aspectos de baixas energias de um modelo cosmológico proposto por Parker em 1999 e com o qual ele tinha trabalhado durante seu pós-doutoramento nos Estados Unidos. A mim, então, foi repassada essa tarefa. No final daquele primeiro semestre de 2008, entretanto, Vanzella decidiu voltar sua atenção à adormecida questão do potencial efetivo negativo. Em suas análises preliminares 
ele percebeu que era possível que houvesse alguma relação entre essa questão e o regime de baixas energias em TQCEC. Por conta dessa suposta relação, passamos a discutir quais seriam a interpretação e as possíveis conseqüências daquele potencial efetivo negativo. Daí, então, foi engendrado o estudo motivo desta tese. Conforme foi se solidificando o nosso entendimento acerca do crescimento ilimitado das flutuações do campo, fomos descartando a possibilidade de o potencial efetivo negativo implicar em algum efeito de criação de partículas. Contudo, no último ano, já contando com a colaboração de A. G. S. Landulfo, mostramos que a estabilização do sistema pode dar origem a um processo de criação de partículas em profusão, confirmando, mas de modo diferente do inicialmente imaginado, as especulações de Matsas.

Os principais resultados que serão apresentados adiante em detalhes constam nas referências $(24,27)$ (veja também as referências $(28,29)$ ). A primeira referência trata do efeito em espaços-tempos bastante gerais, enquanto que a segunda consiste de uma realização do Efeito de Despertar do Vácuo em contextos astrofísicos. A quarta referência é uma versão preliminar do manuscrito sobre a criação de partículas engendrada pelo efeito discutido nesta tese e será seguido, em breve, por uma versão mais extensa e detalhada. Já a última trata de uma outra realização do mecanismo de despertar do vácuo, a saber, no espaço-tempo gerado por uma casca esférica e já se encontra no processo de escrita. Uma eventual aplicação do efeito em cenários cosmológicos ainda se encontra sob investigação. Ao longo da tese referir-me-ei a esse novo efeito como "Efeito de Despertar do Vácuo", termo esse por nós cunhado nas referências mencionadas.

Esta tese está organizada da seguinte forma. Uma vez que é recorrente ao longo do texto o uso de algumas definições do Cálculo em variedades e Geometria Diferencial, no Capítulo 2 faço um apanhado das definições que serão empregadas. O conteúdo desse capítulo está baseado principalmente nas referências (2,4). No Capítulo 3 é apresentada a quantização de um campo escalar real livre obedecendo uma equação linear. Ao longo desse capítulo são usadas algumas definições e resultados de Análise Funcional. Como os tópicos desse tema necessários para transformar a exposição em autocontida são muitos, acrescentá-los faria com que o texto como um todo se desviasse em demasia de seu objetivo 
principal. Sendo assim, decidi por relegar ao leitor a tarefa de recorrer à literatura. Para o tema de Análise Funcional sugiro as referências (30 32). Para completar as discussões preliminares, no Capítulo 4 é apresentado o estudo de algumas propriedades gerais de soluções complexas da equação de campo de KG. No Capítulo 5 inicio a exposição dos resultado originais contidos nesta tese ao apresentar o Efeito de Despertar do Vácuo em espaços-tempos bastante gerais e analisar algumas de suas características. No Capítulo6 mostro que o efeito pode ser despertado na formação de corpos compactos e no espaçotempo gerado por uma casca esférica. O espaço de estados do campo quântico enquanto o vácuo encontra-se "desperto" é estudado com detalhes ao longo do Capítulo 7 . No Capítulo 8 será abordada a relação entre o efeito e fenômenos de excitação de detectores e criação partículas. Por fim, no Capítulo 9, tratarei de questões pertinentes a diversos aspectos do problema.

Ao longo deste texto o sistema de unidades usado é aquele no qual $G=\hbar=c=1$, exceto onde explicitamente mencionado o contrário. A assinatura adotada para métrica é $(-+++)$. O símbolo $i$ é reservado para a unidade imaginária e $\bar{z}$ denota o complexo conjugado de um número complexo $z$. As quantidades $\operatorname{Re} z$ e $\operatorname{Im} z$ denotam, respectivamente, as partes real e imaginária de $z$, enquanto que as c.c. e h.c. abreviam as expressões "complexo conjugado" e "hermitiano conjugado". O símbolo := indica que o lado esquerdo da sentença está definido pelo lado direito. Para vetores, covetores e tensores será utilizada a notação de índice abstrato, como definida em (4). 


\section{Capítulo 2}

\section{Discussões preliminares sobre}

\section{geometria}

4 ferencial que serão usadas ou tacitamente assumidas ao longo do texto, além de algumas definições relativas à decomposição 3+1 de espaços-tempos globalmente hiperbólicos. Algumas definições apresentadas na Seção 2.1. como as espaços-tempos globalmente hiperbólicos, isometria e derivada de Lie, serão particularmente importantes na quantização do campo no Capítulo 3 . Além disso, as manipulações e definições feitas na Seção 2.2 serão empregadas mais adiante no Capítulo 4 para o estudo da equação de KG.

\subsection{Algumas definições}

Seja $\mathcal{M}$ uma variedade diferenciável real, suave e $n$-dimensional. Isso significa que $\mathcal{M}$ é um conjunto com uma coleção de subconjuntos $\left\{\mathcal{O}_{\alpha}\right\}$, elementos de uma topologia $\mathscr{T}$ em $\mathcal{M}$, tal que

(a) cada ponto de $\mathcal{M}$ pertence a pelo menos um dos elementos dessa coleção,

(b) para cada $\mathcal{O}_{\alpha}$ existe um mapa bijetor contínuo e com inversa contínua $\psi_{\alpha}: \mathcal{O}_{\alpha} \rightarrow U_{\alpha}$ 
entre este subconjunto e um aberto $U_{\alpha} \subset \mathbb{R}^{n} \mathrm{e}$

(c) quando $\mathcal{O}_{\alpha} \cap \mathcal{O}_{\beta} \neq \emptyset$, o mapa $\psi_{\beta} \circ \psi_{\alpha}^{-1}$, que leva $\psi_{\alpha}\left[\mathcal{O}_{\alpha} \cap \mathcal{O}_{\beta}\right] \subset U_{\alpha}$ em $\psi_{\beta}\left[\mathcal{O}_{\alpha} \cap \mathcal{O}_{\beta}\right] \subset$ $U_{\beta}$, é infinitamente diferenciável.

Na literatura matemática os mapeamentos $\psi_{\alpha}$ são conhecidos como cartas e seu conjunto, $\left\{\psi_{\alpha}\right\}$, como atlas. Para se evitar que o acréscimo ou retirada de cartas de $\left\{\psi_{\alpha}\right\}$ defina uma nova variedade normalmente impõem-se que o atlas é maximal, i.e., abarca todas as cartas possíveis satisfazendo as condições (b) e (c) acima. As cartas nada mais são do que aquilo que em Física conhecemos como sistema de coordenadas. Ademais, o espaço topológico $(\mathcal{M}, \mathscr{T})$ ainda deve ser Hausdorff - i.e., para $p, q \in \mathcal{M}$ e $p \neq q$ existem $\mathcal{O}_{p}, \mathcal{O}_{q} \in \mathscr{T}$ tais que $p \in \mathcal{O}_{p}$ e $q \in \mathcal{O}_{q}$ e que $\mathcal{O}_{p} \cap \mathcal{O}_{q}=\emptyset$ - e segundo contável - i.e., existe uma coleção contável de abertos de $\mathscr{T}$ tal que todo aberto da topologia pode ser escrito como união de elementos dessa coleção. (Para uma exposição sobre tópicos em Topologia úteis ao Cálculo em variedades, veja a referência (32).) Definida dessa forma, a variedade $\mathcal{M}$ é um objeto que, ao menos localmente, "se assemelha" a $\mathbb{R}^{n}$.

Um vetor tangente $v$ em $p \in \mathcal{M}$ é definido com um mapeamento entre o espaço das funções infinitamente diferenciáveis com suporte em $\mathcal{M}, C^{\infty}(\mathcal{M})$, e o conjunto dos reais que é linear, $v(a f+b g)=a v(f)+b v(g)$, com $a, b \in \mathbb{R}$ e $f, g \in C^{\infty}(\mathcal{M})$ e satisfaz a regra de Leibnitz, $v(f g)=v(f) g(p)+f(p) v(g)$. Essa definição é motivada pela relação unívoca que existe em $\mathbb{R}^{n}$ entre derivada direcional de uma função calculada em um ponto e o conceito usual de vetores como uma ênupla de números reais. Por conta da definição, o conjunto de todos os vetores em $p \in \mathcal{M}, V_{p}$, tem naturalmente a estrutura de espaço vetorial e, em particular, é possível provar (veja, e.g., o Teorema 2.2.1 da referência (4)) que $V_{p}$ tem a mesma dimensão da variedade. Dada uma carta $\psi$ sobre um subconjunto $\mathcal{O} \subset \mathcal{M}$, é sempre possível definir em $V_{p}$, com $p \in \mathcal{O}$, os vetores $X_{\mu}(f):=\left.\partial_{\mu}\left(f \circ \psi_{\alpha}^{-1}\right)\right|_{p}$, com $\mu=0,1, \ldots, n-1$, denominados vetores coordenados. Uma vez definido o espaço tangente a $\mathcal{M}$ no ponto $p$, é possível definir o espaço dual - também chamado de espaço cotangente - associado, $V_{p}^{*}$, como a coleção dos mapeamentos lineares $\omega: V_{p} \rightarrow \mathbb{R}$ que também é um espaço vetorial se definida as operações de adição e multiplicação por 
escalar da forma usual. A dimensão de $V_{p}^{*}$ é igual à dimensão de $V_{p}$ e que existe uma relação unívoca entre os elementos de $V_{p}$ e $V_{p}^{* *}$, ou seja, o dual do espaço dual pode ser identificado com o próprio espaço tangente. Uma vez de posse do espaço tangente e seu dual é possível definir o espaço dos tensores de posto $(m, n)$ em $p \in \mathcal{M}$ como o conjunto dos mapeamentos multilineares

$$
T: \underbrace{V_{p}^{*} \times \cdots \times V_{p}^{*}}_{m} \times \underbrace{V_{p} \times \cdots \times V_{p}}_{n} \rightarrow \mathbb{R}
$$

em outras palavras, dados $m$ covetores e $n$ vetores em $p \in \mathcal{M}$, o tensor $T$ associa a eles um número real. Da mesma forma que para $V_{p}^{*}$, o espaço de todos os tensores de posto $(m, n)$ em $p \in \mathcal{M}, \mathcal{T}_{p}(m, n)$, pode ser feito em um espaço vetorial ao definirmos a adição e a multiplicação por escalar desses mapas do modo usual. Dada uma base $\left\{v_{\alpha}\right\}$ para $V_{p}$ e $\left\{\omega^{\beta}\right\}$ para $V_{p}^{*}$, que por definição satisfazem $\omega^{\mu}\left(v_{\nu}\right)=\delta^{\mu}{ }_{\nu}$, é possível definir a operação conhecida como contração dos índices de um tensor como um mapeamento entre $\mathcal{T}_{p}(m, n)$ e $\mathcal{T}_{p}(m-1, n-1)$ fazendo $\sum_{\sigma} T\left(w_{1}, \ldots, \omega^{\sigma}, \ldots, w_{m} ; u_{1}, \ldots, v_{\sigma}, \ldots, u_{n}\right)$.

Um campo tensorial de particular importância no que segue é o tensor métrico ou simplesmente métrica. Um tensor $g$ de posto $(0,2)$ é dito ser uma métrica se é simétrico, $g\left(v_{1}, v_{2}\right)=g\left(v_{2}, v_{1}\right)$, e não-degenerado, $g\left(v, v_{1}\right)=0$ para todo $v$ se, e somente se, $v_{1}$ é o vetor nulo. No caso da geometria riemanniana, onde a métrica define naturalmente um produto interno bona fide nos espaços tangentes à variedade, essa definição vai ao encontro da noção intuitiva de que a métrica está atrelada à distância entre pontos infinitesimalmente próximos, uma vez que, da forma com que foram definidos acima, vetores guardam relação com deslocamentos infinitesimais. O tensor métrico naturalmente define um mapeamento entre o espaço tangente e seu dual, haja visto que, dado $v \in V_{p}$, $g(\cdot, v)$ define um mapeamento entre entre $V_{p}$ e $\mathbb{R}$. Por conta da definição, dado um ponto da variedade é sempre possível encontrar uma base de $V_{p}$ que diagonaliza $g$. Como a transmissão de sinais em Física é usualmente descrita por equações hiperbólicas, aqui estamos interessados em métricas lorentzianas, isto é, métricas que quando diagonalizadas em um dado ponto de $\mathcal{M}$ têm a forma diag $(-1,+1,+1,+1)$. No que segue o par $(\mathcal{M}, g)$ constituído por uma variedade diferenciável, suave e real quadridimensional $\mathcal{M}$ e uma 
métrica lorentziana $g$ será denominado espaço-tempo. Métricas lorentzianas permitem a classificação dos vetores do espaço tangente em três grupos: vetores tipo tempo, espaço e luz. Se $v^{a}$ é tipo tempo, então $g_{a b} v^{a} v^{b}<0$, se tipo espaço, então $g_{a b} v^{a} v^{b}>0$ e se tipo luz, então $g_{a b} v^{a} v^{b}=0$. Da mesma forma, se os vetores tangentes a uma curva $\gamma: \mathbb{R} \rightarrow \mathcal{M}$ são tipo espaço, então a curva a dita ser tipo espaço e assim por diante. O fato de objetos dotados de inércia não poderem localmente viajar mais rápido do que a velocidade da luz implica que as curvas por eles descritas são sempre tipo tempo e sua quadrivelocidade $u^{a}$ é definida de modo a satisfazer $g_{a b} u^{a} u^{b}=-1$. Pelo mesmo motivo, pontos do espaço-tempo que guardam relação de causalidade entre si só podem estar ligados por curvas tipo tempo ou luz e por isso essas curvas levam o nome de causais.

Um operador derivada ou derivada covariante $\nabla$ é definido em $\mathcal{M}$ como um mapeamento entre $\mathcal{T}_{p}(m, n)$ e $\mathcal{T}_{p}(m, n+1)$ que é linear, satisfaz a regra de Leibnitz, comuta com a operação de contração, é consistente com a noção de que vetores estão relacionados à derivadas direcionais de funções suaves sobre $\mathcal{M}, t(f)=t^{a} \nabla_{a} f$, e tem torção nula, isto é, $\nabla_{a} \nabla_{b} f=\nabla_{b} \nabla_{a} f$ 円 Dados quaisquer dois operadores derivada com as propriedades acima é possível mostrar, estudando-se a forma com que $\nabla$ atua em funções, vetores e covetores, que eles diferem de uma maneira que pode ser codificada em um tensor de posto $(1,2)$. Uma vez definida uma métrica sobre $\mathcal{M}$, a condição $\nabla_{c} g_{a b}=0$ seleciona univocamente um operador derivada e, conseqüentemente, a forma de $\Gamma_{b c}^{a}$, depois de fixado o sistema de coordenadas (veja, e.g., o Teorema 3.1.1 da referência (4)). O critério por detrás dessa escolha é o do transporte paralelo. Em princípio, dados $p, q \in \mathcal{M}$, não há qualquer tipo de identificação privilegiada entre $V_{p}$ e $V_{q}$ e, em particular, não é possível dizer que um vetor em $p$ é paralelo a outro em $q$. Entretanto, uma vez definido um operador derivada, pode-se estabelecer uma noção de paralelismo entre vetores em pontos distintos recorrendo-se a noção intuitiva de que se um vetor é paralelamente transportado ao longo de uma curva em $\mathcal{M}$ ele permanece "o mesmo". Em outras palavras, sendo $t^{a}$ o vetor tangente à curva, se $v^{b}$ é transportado paralelamente a si mesmo então $t^{a} \nabla_{a} v^{b}=0$. Além disso, se um par de vetores são paralelamente transportados ao longo de uma curva então seu produto

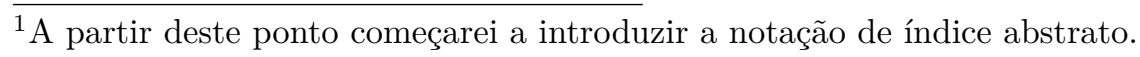


interno, como definido pela métrica, deve ser conservado. Essa última imposição leva a $\nabla_{c} g_{a b}=0$.

Uma vez selecionado o operador derivada através do critério do transporte paralelo de vetores, defini-se o tensor de Riemann por

$$
R_{a b c}{ }^{d} \omega_{d}:=\nabla_{a} \nabla_{b} \omega_{c}-\nabla_{b} \nabla_{a} \omega_{c}
$$

onde $\omega_{c}$ é um campo covetorial arbitrário. O tensor de Riemann é responsável por descrever a curvatura associada à geometria definida pela métrica $g_{a b}$. A idéia por detrás dessa definição é a seguinte. Um dos efeitos de uma geometria curva é o fato de que vetores paralelamente transportado a si mesmos ao longo de caminhos fechados não correspondem ao vetor inicial quando uma volta é completada. O tensor de Riemann dá uma medida desse efeito. No contexto da RG, duas contrações do tensor de Riemann são particularmente importantes. A primeira,

$$
R_{a b}:=R_{a c b}^{c},
$$

define o chamado tensor de Ricci e a segunda,

$$
R:=R_{a}^{a}=R_{a c}^{a c},
$$

define o escalar de curvatura ou de Ricci. No contexto da RG, sua importância está no fato de comparecerem no lado esquerdo da equação de Einstein sem constante cosmológica:

$$
R_{a b}-\frac{1}{2} R g_{a b}=8 \pi T_{a b}
$$

onde $T_{a b}$ é o tensor energia momentum associado ao conteúdo de matéria e outros campos que compõem o sistema gravitacional considerado. Esse tensor será definido mais adiante para caso particular de um campo escalar.

Ao longo da exposição que se seguirá nos próximos capítulos recorrerei em alguns momentos a espaços-tempos com simetrias, de modo que faz-se mister definir os conceitos de difeomorfismo e isometria. Sejam $\mathcal{M}$ e $\mathcal{N}$ duas variedades, $\phi: \mathcal{M} \rightarrow \mathcal{N}$ um mapa e $f: \mathcal{N} \rightarrow \mathbb{R}$ uma função, ambos suaves, i.e., ambos infinitamente diferenciá- 
veis segundo a estrutura diferencial das variedades $\mathcal{M}$ e $\mathcal{N}$. Então $\phi$ "puxa para trás" a função $f$ ao definirmos $f \circ \phi: \mathcal{M} \rightarrow \mathbb{R}$. Através de $\phi$ é possível estabelecer uma relação entre o espaço tangente a $\mathcal{M}$ no ponto $p$ e o espaço tangente a $\mathcal{N}$ no ponto $\phi(p)$. Para tanto, defina $\phi^{*}: V_{p} \rightarrow V_{\phi(p)}$ por $\left(\phi^{*} v\right)(f):=v(f \circ \phi)$, o pull back map. Da mesma forma, é possível definir $\phi_{*}: V_{\phi(p)}^{*} \rightarrow V_{p}^{*} \operatorname{como}\left(\phi_{*} \omega\right)(v):=\omega\left(\phi^{*} v\right)$, o push forward map. Uma vez definidos os mapeamentos $\phi^{*}$ e $\phi_{*}$, respectivamente, para vetores e covetores é possível ir adiante e definir mapeamentos semelhantes para tensores de posto $(0, n),\left(\phi_{*} T\right)\left(v^{1}, \ldots, v^{n}\right):=T\left(\phi^{*} v^{1}, \ldots, \phi^{*} v^{n}\right)$, e para tensores de posto $(m, 0)$, $\left(\phi^{*} T\right)\left(\omega_{1}, \ldots, \omega_{m}\right):=T\left(\phi_{*} \omega_{1}, \ldots, \phi_{*} \omega_{m}\right)$. Entretanto, para tensores mais gerais, de posto $(m, n)$, não é possível fazer o mesmo sem assumir propriedades extras para o mapa $\phi$.

Um mapa suave $\phi: \mathcal{M} \rightarrow \mathcal{N}$ é dito ser um difeomorfismo se é bijetor e se sua inversa também é suave. A bijeção das derivadas de $\phi$ implica que $\mathcal{M}$ e $\mathcal{N}$ devem ter a mesma dimensão. Uma vez que $\phi^{-1}$ leva $\mathcal{N}$ em $\mathcal{M}$, tem-se que $\left[\phi^{-1}\right]^{*}: V_{\phi(p)} \rightarrow V_{p}$; Então, para tensores de posto $(m, n)$ é possível definir

$$
\left(\phi^{*} T\right)\left(\omega_{1}, \ldots, \omega_{m} ; v_{1}, \ldots, v_{n}\right):=T\left(\phi_{*} \omega_{1}, \ldots, \phi_{*} \omega_{m} ;\left[\phi^{-1}\right]^{*} v_{1}, \ldots,\left[\phi^{-1}\right]^{*} v_{m}\right)
$$

É também possível estender $\phi_{*}$ a todos os tensores, entretanto, uma vez que $\phi_{*}=\left[\phi^{-1}\right]^{*}$, $\phi^{*}$ e $\left[\phi^{-1}\right]^{*}$ já são suficientes. Agora, se $\phi: \mathcal{M} \rightarrow \mathcal{M}$ é um difeomorfismo, então em qualquer ponto de $\mathcal{M}$ é possível comparar o campo tensorial $T$ com o que resulta do mapeamento $\phi^{*} T$. Se $\phi^{*} T=T$, então $\phi$ é dito ser uma transformação de simetria do tensor $T$. Se o tensor em questão é a métrica $g$, então $\phi$ tal que $\phi^{*} g=g$ é dito ser uma isometria.

Um grupo de difeomorfismos a um parâmetro é um mapeamento suave $\phi_{t}: \mathbb{R} \times \mathcal{M} \rightarrow$ $\mathcal{N}$ tal que para $t_{0} \in \mathbb{R}$ fixo, $\phi_{t_{0}}: \mathcal{M} \rightarrow \mathcal{N}$ é um difeomorfismo e para $t_{0}, t_{1} \in \mathbb{R}, \phi_{t}$ satisfaz $\phi_{t_{0}} \circ \phi_{t_{1}}=\phi_{t_{0}+t_{1}}$. A $\phi_{t}$ é possível associar um campo vetorial $\varkappa^{a}$ da seguinte forma. Fixado $p \in \mathcal{M}, \phi_{t}(p)$ define uma curva em $\mathcal{M}$ parametrizada por $t$; o campo vetorial $\varkappa^{a}$ será, então, formado pelos vetores tangentes a essas curvas. Com o auxílio de $\phi_{t}$ é possível definir o seguinte mapeamento linear de $\mathcal{T}_{p}(m, n)$ em si mesmo: dado um campo tensorial 
$T$ defina $£_{\varkappa}$ como

$$
£_{\varkappa} T:=\lim _{t \rightarrow 0} \frac{\phi_{-t}^{*} T-T}{t},
$$

a derivada de Lie de $T$ com respeito ao campo vetorial $\varkappa^{a}$. Explorando sua definição e, em particular, que para uma função $f$ a atuação da derivada de Lie se dá de forma que $£_{\varkappa} f=\varkappa(f)$, é possível mostrar que, em termos da derivada covariante,

$$
\begin{gathered}
\sum_{\varkappa}^{T_{b_{1} \ldots b_{n}} T_{1} \ldots a_{m}}=\varkappa^{c} \nabla_{c} T_{b_{1} \ldots b_{n}}^{a_{1} \ldots a_{m}}-\sum_{i=1}^{m} T_{b_{1} \ldots b_{n}}^{a_{1} \ldots a_{i-1} c a_{i+1} \ldots a_{m}} \nabla_{c} \varkappa^{a_{i}} \\
+\sum_{i=1}^{n} T_{b_{1} \ldots b_{i-1} c b_{i+2} \ldots b_{n}}^{a_{1} \ldots a_{m}} \nabla_{b_{i}} \varkappa^{c}
\end{gathered}
$$

o que implica, no caso em que o campo tensorial em questão é a métrica do espaço-tempo, que

$$
£_{\varkappa} g_{a b}=\nabla_{a} \varkappa_{b}+\nabla_{b} \varkappa_{a} .
$$

Se para cada $t \in \mathbb{R}$ o grupo de difeomorfismos a um parâmetro $\phi_{t}$ também é uma isometria, é evidente, pela Eq. (2.1.7), que $£_{\varkappa} g=0$, o que implica que $\varkappa^{a}$ satisfaz

$$
\nabla_{a} \varkappa_{b}+\nabla_{b} \varkappa_{a}=0
$$

A Eq. 2.1.11 é conhecida como equação de Killing e o campo vetorial que a satisfaz, $\varkappa^{a}$, como campo de Killing.

Uma vez que nesta tese a equação de onda é um elemento importante, desejo focar atenção nos espaços-tempos para os quais pode-se garantir que a dinâmica clássica do sistema tenha uma formulação bem-definida em relação às condições iniciais em toda a variedade. Para tanto é necessário, em primeiro lugar, restringir-se a espaços-tempos temporalmente orientáveis. Pela forma com que foi construído, o espaço tangente à variedade $\mathcal{M}$ no ponto $p$ pode ser identificado ao espaço-tempo de Minkowski $\left(\mathbb{R}^{4}, \eta\right)$, onde a métrica, definida por $R_{a b c}{ }^{d}=0$, pode ser colocada na forma $\eta=\operatorname{diag}(-1,+1,+1,+1)$. Com essa identificação em mente, defini-se o cone de luz em $p$ como sendo o cone de luz que passa pela origem de $V_{p}$. Um espaço-tempo é dito orientável temporalmente se é possível designar cada metade do cone de luz em $p$ como "passado" e "futuro" de modo que essa designação seja feita continuamente a medida em que se varia $p$. Seja $\Sigma \subset \mathcal{M}$ um 
conjunto fechado e acronal, i.e., nenhum par de pontos $p, q \in \Sigma$ pode estar conectado por uma curva tipo tempo. Defini-se, então, o domínio de dependência de $\Sigma$ como o conjunto $D(\Sigma)$ dos pontos da variedade tais que toda curva causal inestendível para o passado ou futuro - ou seja, uma curva tipo tempo ou tipo luz que não "pára" na vizinhança de nenhum ponto de $\mathcal{M}$ - que passa por $p$ intersecta $\Sigma$ uma vez. O domínio de dependência de uma região acronal $\Sigma$, ou seja, uma região de $(\mathcal{M}, g)$ na qual nenhum par de pontos pode ser ligado por uma curva tipo tempo, é aquela região do espaço-tempo formada por pontos que podem ser alcançadas por sinais luminosos ou partículas maciças emitidas em $\Sigma$ ou pontos dos quais foram emitidos sinais luminosos ou partículas que atingirão $\Sigma$. Se $D(\Sigma)=\mathcal{M}$, então $\Sigma$ é dito ser uma superfície de Cauchy para o espaço-tempo $(\mathcal{M}, g)$. É possível mostrar que se $\Sigma$ é uma superfície de Cauchy, então $\Sigma$ é uma hiperfície tridimensional contínua (veja, e.g., o Teorema 8.3.1 da referência (4)); se $(\mathcal{M}, g)$ admite uma superfície de Cauchy então o espaço-tempo é dito ser globalmente hiperbólico. Em particular, se $(\mathcal{M}, g)$ é globalmente hiperbólico, então é sempre possível escolher uma "função tempo" suave $t$ e uma foliação $\left\{\Sigma_{t}\right\}_{t \in \mathbb{R}} \equiv \mathbb{R} \times \Sigma$, de modo que cada ponto de $\mathcal{M}$ pertence a somente uma hiperfície $\Sigma_{t}$ e que, para $t$ fixo, $\Sigma_{t} \equiv\{t\} \times \Sigma$ é uma superfície de Cauchy (33). Um espaço-tempo globalmente hiperbólico é, então, aquele no qual toda sua história futura (passada) pode ser predita (retrocedida) a partir de condições em um instante de tempo representado por uma superfície de Cauchy.

Outros conceitos que serão freqüentemente usado ao longo desta tese são o de observador e o de família de observadores. Um observador é definido como uma curva tipo tempo $\gamma$ cuja quadrivelocidade é, em todos os pontos de $\gamma$, direcionada para o futuro. Já uma família de observadores consiste de uma família de curvas direcionadas para o futuro tal que por cada ponto da variedade passa somente uma curva. 


\subsection{Decomposição $3+1$}

Seja $(\mathcal{M}, g)$ um espaço-tempo globalmente hiperbólico para o qual foi escolhida uma certa foliação. Por $n^{a}$ denoto um campo vetorial suave tipo tempo apontando para o futuro tal que em qualquer ponto $n^{a}$ é um vetor unitário e normal a cada hiperfície pertencente à foliação escolhida. O tensor métrico $h_{a b}$ induzido em cada $\Sigma_{t}$ é definido por

$$
h_{a b}:=g_{a b}+n_{a} n_{b}
$$

Em particular, isso implica que

$$
h_{a b} n^{b}=0
$$

Daí segue que o tensor $h^{a b}$, o inverso de $h_{a b}$, é dado por

$$
h^{a b}:=g^{a b}+n^{a} n^{b}
$$

pois

$$
h^{a b} h_{b c}=\delta_{c}^{a}
$$

quando restrito ao espaço de tensores sobre $\Sigma_{t}$.

A derivada covariante consistente com a métrica $h_{a b}$ é definida por

$$
D_{c} T_{b_{1} \ldots b_{m}}^{a_{1} \ldots a_{n}}:=h_{d_{1}}^{a_{1}} \ldots h_{d_{n}}^{a_{n}} h_{b_{1}}^{e_{1}} \ldots h_{b_{m}}^{e_{m}} h_{c}^{f} \nabla_{f} T_{e_{1} \ldots e_{m}}^{d_{1} \ldots d_{n}} .
$$

Isso é verdade, haja visto que $D_{c}$ satisfaz todos os requerimentos de um operador derivada e $D_{c} h_{a b}=0$. Por fim, define-se a curvatura extrínseca de $\Sigma_{t}, K_{a b}$, e seu traço $K$, por

$$
K_{a b}:=h_{a}^{c} \nabla_{c} n_{b}
$$

e

$$
K:=g^{a b} K_{a b}
$$

Para uso futuro vou também definir o campo a ${ }^{a}$ como

$$
\mathrm{a}^{a}:=n^{b} \nabla_{b} n^{a} .
$$


Observadores seguindo as linhas integrais do campo $n^{a}$ — isto é, seguindo uma família de curvas com a propriedade de que uma, e somente uma, curva passa por cada ponto $p \in \mathcal{M}$ e que o vetor tangente à curva que passa em $p$ é igual a $\left.n^{a}\right|_{p}$ - têm como quadriaceleração o vetor $\mathrm{a}^{a}$.

O espaço-tempo é dito ser estacionário se possuir uma isometria temporal — ou seja, gerada por um campo vetorial de Killing tipo tempo. Um espaço-tempo estacionário é também estático se existir uma foliação à qual o campo vetorial que gera a isometria é ortogonal. Pelo teorema de Frobenius (veja, e.g., o Apêndice B da referência (4)), a existência dessa foliação é equivalente à condição de que

$$
\varkappa_{[a} \nabla_{b} \varkappa_{c]}=0
$$

Os colchetes na Eq. 2.2.9 indicam a anti-simetrização dos índices por eles compreendidos. O campo de Killing $\varkappa^{a}$ pode ser empregado como vetor coordenado na direção temporal, o que implica, da equação de Killing, que

$$
\frac{\partial}{\partial t} g_{\alpha \beta}=0
$$

quando o espaço-tempo é estático. Isso pode ser feito, uma vez que as linhas integrais de $\varkappa^{a}$ interseccionam $\Sigma_{t}$ somente em um ponto. Para essa foliação em particular, teremos que

$$
n^{a}:=\frac{\varkappa^{a}}{\|\varkappa\|}
$$

onde $\|\varkappa\|:=\sqrt{-\varkappa^{c} \varkappa_{c}}$. Isso implica que

$$
K_{a b}=0
$$

e que o campo vetorial a ${ }^{a}$ pode ser escrito da seguinte forma:

$$
\mathrm{a}^{a}=\nabla^{a} \ln \|\varkappa\| .
$$

Por fim, no caso de um espaço-tempo estático é dito ser esfericamente simétrico se seu grupo de isometria contem um subgrupo que é isomorfo ao grupo $\mathrm{SO}(3)$ e cujas órbitas, i.e., curvas construídas pela ação da isometria em pontos da variedade, geram superfícies 
esféricas bidimensionais. Nesse caso a variedade $\mathcal{M}$ é homeomorfa à variedade $\mathbb{R} \times \mathbb{R}_{+} \times \mathcal{S}^{2}$ (identificando-se entre si todos os pontos da fronteira). Assim, pode ser escolhida uma foliação para todo $\Sigma_{t}$ em termos de superfícies esféricas parametrizadas por um $s \in \mathbb{R}_{+}$. A parametrização das superfícies esféricas é arbitrária e é usualmente escolhida de modo que $s=r:=(A / 4 \pi)^{1 / 2}$, onde $A$ é a área da superfície esférica. Como essas superfícies são geradas por campos de Killing, é possível escolher um campo vetorial $\left(e_{r}\right)^{a}$, definido por

$$
\left(e_{r}\right)^{a}:=\frac{\nabla^{a} r}{\left(\nabla_{c} r \nabla^{c} r\right)^{1 / 2}},
$$

de modo que, em todo ponto de $\mathcal{M},\left(e_{r}\right)^{a}$ é perpendicular a esses campos. Se $\left(e_{r}\right)^{a}$ é um campo normalizado, pode-se induzir uma métrica sobre as superfícies esféricas por

$$
s_{a b}:=h_{a b}-\left(e_{r}\right)_{a}\left(e_{r}\right)_{b} .
$$

Denotarei por $\Theta_{a}$ o operador derivada consistente com a métrica $s_{a b}$ e definido por

$$
\Theta_{c} T_{b_{1} \ldots b_{m}}^{a_{1} \ldots a_{n}}:=s^{a_{1}}{ }_{d_{1}} \ldots s^{a_{n}}{ }_{d_{n}} s^{e_{1}}{ }_{b_{1}} \ldots s^{e_{m}}{ }_{b_{m}} s^{f}{ }_{c} D_{f} T_{e_{1} \ldots e_{m}}^{d_{1} \ldots d_{n}} .
$$

Por fim, é possível definir a curvatura extrínseca da foliação de cada $\Sigma_{t}$ como

$$
S_{a b}:=s_{a}{ }^{c} D_{c}\left(e_{r}\right)_{b}
$$

sendo que o traço é dado por

$$
S:=s^{a b} D_{a}\left(e_{r}\right)_{b}
$$




\section{Capítulo 3}

\section{Formalismo de quantização canônica}

\section{para campos livres}

W ESTE capítulo apresento uma formulação, baseada principalmente na referência (7), ser uma construção muito bonita, ela clarifica algumas questões tratadas mais adiante como, por exemplo, a quantização do campo escalar real quando da presença de modos que crescem exponencialmente. O formalismo é inicialmente apresentado na Seção 3.1 para espaços-tempos bastante gerais e depois particularizado para espaços-tempos que possuem uma isometria temporal na Seção 3.2. A construção nesse último contexto é importante para o propósito desta tese, haja visto que amiúde assumirei, como uma idealização útil, que a geometria de fundo é assintoticamente estática no passado e no futuro. Em seguida, na Seção 3.3 defino a transformação de Bogoliubov, uma ferramenta muito útil no contexto de TQCEC e que será empregada nos capítulos seguintes. Por fim, na Seção 3.4 discorro sobre o tensor energia-momentum para o campo quântico. Esse observável será fundamental para a análise das possíveis conseqüências do efeito motivo deste estudo. 


\subsection{Quantização em espaços-tempos gerais}

O formalismo de quantização canônica é usualmente abordado começando-se pela ação clássica que define o sistema de campos. No que concerne ao problema exposto nesta tese, estou interessado na ação de um campo escalar real de KG — de massa $m>0$, com acoplamento não-mínimd ${ }^{1} \xi \in \mathbb{R}$ e definido sobre o espaço-tempo globalmente hiperbólico $(\mathcal{M}, g)$ - definida por

$$
S_{\mathrm{KG}}[\phi]:=-\frac{1}{2} \int_{\mathcal{M}} \sqrt{-g} d^{4} x\left[g^{a b} \nabla_{a} \phi \nabla_{b} \phi+\left(m^{2}+\xi R\right) \phi^{2}\right]
$$

onde $\sqrt{-g} d^{4} x$ denota o elemento de volume do espaço-tempo. Variando $S_{\mathrm{KG}}$ com relação a $\phi$ segue a respectiva equação de Euler-Lagrange:

$$
-\nabla_{a} \nabla^{a} \phi+\left(m^{2}+\xi R\right) \phi=0
$$

a equação de KG. Para continuar é necessário definir uma coordenada temporal t . Como já mencionado na Seção 2.1, para um espaço-tempo globalmente hiperbólico isso implica na escolha de uma foliação $\left\{\Sigma_{t}\right\}_{t \in \mathbb{R}}$, onde cada hiperfície $\Sigma_{t}$ é uma superfície de Cauchy. Uma vez escolhida a coordenada $t$, é possível definir o momentum canonicamente conjugado ao campo, $\pi$, através da variação da ação do campo com respeito a $\dot{\phi}:=\partial_{t} \phi$ :

$$
\pi:=\frac{\delta S_{\mathrm{KG}}[\phi]}{\delta \dot{\phi}}=\sqrt{h} n^{a} \nabla_{a} \phi
$$

onde $h$ denota o determinante da métrica espacial definida na Eq. 2.2.1). Satisfeita a hipótese de que $(\mathcal{M}, g)$ é globalmente hiperbólico, o problema de se obter soluções da equação de KG Eq. (3.1.2) em termos de condições iniciais é bem-posto em todo o espaço-tempo. Mais especificamente, dado um par de funções infinitamente diferenciáveis $\left(\phi_{0}, \pi_{0}\right)$, com domínio em alguma hiperfície $\Sigma_{t_{0}}$, existe uma única solução definida em todo o espaço-tempo para a Eq. (3.1.2) tal que $\phi=\phi_{0}$ e $\pi=\pi_{0}$ quando restritos a $\Sigma_{t_{0}}$

\footnotetext{
${ }^{1} \mathrm{O}$ campo $\phi$ é dito estar minimamente acoplado ao campo gravitacional quando $\xi=0$. Essa nomenclatura é inspirada na regra de acoplamento mínimo em Eletrodinâmica. Quando, em 4 dimensões, $\xi=1 / 6$, o acoplamento é dito conforme. Isso, pois sob transformações conformes, i.e., para um reescalonamento do tensor métrico - que pode depender do ponto do espaço-tempo - a forma da equação de campo para $m=0$ é preservada.
} 
e para qualquer conjunto $S \subset \Sigma_{t_{0}}$ fechado a solução $\phi$, quando restrita a $D(S)$, depende somente das condições iniciais em $S$; ademais, a solução é suave e depende continuamente das condições iniciais (veja, e.g., as Proposições 7.4.5 e 7.4 .7 da referência (2)).

Para expor o procedimento de quantização canônica, é necessário, em primeiro lugar, fixar a teoria clássica. No caso da Eq. (3.1.2), isso consiste em definir precisamente o espaço de fase $\Gamma$ para o campo $\phi$ ou, em outras palavras, as propriedades que deseja-se que sejam satisfeitas pelas funções que servirão de condições iniciais. No caso de um espaçotempo arbitrário é conveniente escolher os elementos do par $\left(\phi_{0}, \pi_{0}\right)$ como elementos do conjunto de funções suaves e de suporte compacto em $\Sigma_{t_{0}}, C_{0}^{\infty}\left(\Sigma_{t_{0}}\right)$ :

$$
\Gamma=\left\{\left(\phi_{0}, \pi_{0}\right) \mid \phi_{0}: \Sigma_{t_{0}} \rightarrow \mathbb{R}, \pi_{0}: \Sigma_{t_{0}} \rightarrow \mathbb{R} ; \phi_{0}, \pi_{0} \in C_{0}^{\infty}\left(\Sigma_{t_{0}}\right)\right\}
$$

Com isso defini-se $\mathscr{S}$ como o espaço de soluções da Eq. 3.1.2 que, quando restritas a $\Sigma_{t_{0}}$, são elementos de $\Gamma$. Do fato de que há uma relação unívoca entre as condições iniciais e as soluções da equação de campo, segue que $\mathscr{S}$ pode ser identificado com $\Gamma$. Ademais, é possível definir uma estrutura bilinear anti-simétrica $\Omega$ sobre $\mathscr{S}$ (ou sobre o espaço de fase) por

$$
\Omega\left(\psi_{1}, \psi_{2}\right):=\int_{\Sigma_{t}}\left(\pi_{1} \phi_{2}-\pi_{2} \phi_{1}\right) d^{3} x
$$

onde $\psi:=(\phi, \pi) ; \Omega$ é dito ser uma forma simplética sobre $\mathscr{S}$ se for não-degenerada. O lado direito da Eq. 3.1.5 independe da escolha da hiperfície $\Sigma_{t}$, posto que é conservado pela dinâmica. O parêntesis de Poisson para $\Omega$, fixados $\psi_{1}$ e $\psi_{2}$, fica:

$$
\begin{aligned}
\left\{\Omega\left(\psi_{1}, \psi_{3}\right), \Omega\left(\psi_{2}, \psi_{3}\right)\right\}:= & \int_{\Sigma_{t}}\left[\frac{\delta}{\delta \phi_{3}} \Omega\left(\psi_{1}, \psi_{3}\right) \frac{\delta}{\delta \pi_{3}} \Omega\left(\psi_{2}, \psi_{3}\right)\right. \\
& \left.-\frac{\delta}{\delta \pi_{3}} \Omega\left(\psi_{1}, \psi_{3}\right) \frac{\delta}{\delta \phi_{3}} \Omega\left(\psi_{2}, \psi_{3}\right)\right] d^{3} x \\
= & -\Omega\left(\psi_{1}, \psi_{2}\right),
\end{aligned}
$$

onde usei que

$$
\frac{\delta}{\delta \phi_{3}} \Omega\left(\psi_{1}, \psi_{3}\right)=\pi_{1}
$$


e que

$$
\frac{\delta}{\delta \pi_{3}} \Omega\left(\psi_{1}, \psi_{3}\right)=-\phi_{1}
$$

Com a teoria clássica definida pode-se, então, atacar a questão de como levar-se a cabo a quantização do campo escalar. A quantização consiste na construção de uma representação irredutível para os operadores $\hat{W}(\psi)$, com $\psi \in \mathscr{S}$, tal que eles sejam unitários, variem continuamente em função de $\psi$ na topologia forte (para a definição do conceito de strong topology operator veja, e.g., a referência (30)) e que satisfaçam

$$
\hat{W}\left(\psi_{1}\right) \hat{W}\left(\psi_{2}\right)=e^{\frac{i}{2} \Omega\left(\psi_{1}, \psi_{2}\right)} \hat{W}\left(\psi_{1}+\psi_{2}\right)
$$

$\mathrm{e}$

$$
\hat{W}^{\dagger}(\psi)=\hat{W}(-\psi)
$$

as relações de Weyl. Escritas dessa forma, as relações de Weyl equivalem à prescrição de quantização canônica aplicada ao parêntesis de Poisson definido na Eq. (3.1.6). De fato, se denotado por $\hat{\Omega}(\psi, \cdot)$, o gerador de $\hat{W}(\psi)$ satisfaz

$$
\left[\hat{\Omega}\left(\psi_{1}, \cdot\right), \hat{\Omega}\left(\psi_{2}, \cdot\right)\right]=-i \Omega\left(\psi_{1}, \psi_{2}\right) \hat{1}
$$

Do ponto de vista matemático, as relações de Weyl Eq. 3.1.8 são preferidas sobre o comutador da Eq. 3.1.9) pelo fato de ser tecnicamente mais simples definir a composição dos operadores limitados $\hat{W}$ do que a dos operadores não-limitados $\left.\hat{\Omega}(\psi, \cdot)\right|^{2}$ Os geradores $\hat{\Omega}(\psi, \cdot)$, por sua vez, podem ser identificados com o operador campo usual por

$$
\hat{\Omega}(\psi, \cdot)=\int_{\Sigma_{t}}(\pi \hat{\phi}-\phi \hat{\pi}) d^{3} x
$$

se, no sentido de distribuição, $\hat{\phi}$ e $\hat{\pi}$ satisfazem as mesmas equações que $\phi$ e $\pi$ e

$$
\begin{aligned}
& {\left.[\hat{\phi}(x), \hat{\phi}(y)]\right|_{\Sigma_{t}}=0,} \\
& {\left.[\hat{\pi}(x), \hat{\pi}(y)]\right|_{\Sigma_{t}}=0,}
\end{aligned}
$$

${ }^{2} \mathrm{O}$ fato de que os operadores $\hat{\Omega}(\psi, \cdot)$ não são limitados pode ser mostrado através da relação de comutação por eles satisfeita. 
e

$$
\left.[\hat{\phi}(x), \hat{\pi}(y)]\right|_{\Sigma_{t}}=i \delta_{\Sigma_{t}}(x, y) \hat{1}
$$

onde $\delta_{\Sigma_{t}}(x, y)$ é a distribuição delta definida sobre $\Sigma_{t}$.

No contexto de campos, a representação para as relações de Weyl Eq. (3.1.8) é construída de sorte que os operadores $\hat{\Omega}(\psi, \cdot)$ são escritos em termos de operadores de criação e destruição atuando sobre um espaço de Fock $\mathscr{F}_{\mathrm{s}}(\mathscr{H})$. Dado um espaço de Hilbert $\mathscr{H}$, defini-se $\mathscr{F}_{\mathrm{s}}(\mathscr{H})$ como

$$
\mathscr{F}_{\mathrm{s}}(\mathscr{H}):=\mathbb{C} \oplus \mathscr{H} \oplus\left(\mathscr{H} \otimes_{\mathrm{s}} \mathscr{H}\right) \oplus\left(\mathscr{H} \otimes_{\mathrm{s}} \mathscr{H} \otimes_{\mathrm{s}} \mathscr{H}\right) \oplus \ldots,
$$

onde $\oplus$ denota a soma direta e $\otimes_{\mathrm{s}}$ o produto tensorial simetrizado (veja, e.g., a referência (30)) 3 $^{3}$ O problema que se coloca, então, é o de como definir o espaço de Hilbert $\mathscr{H}$. Isso pode ser feito da seguinte forma. Suponha que exista sobre $\mathscr{S}$ um produto interno $\mu: \mathscr{S} \times \mathscr{S} \rightarrow \mathbb{R}$ que satisfaça, para todo $\psi \in \mathscr{S}$,

$$
\mu\left(\psi_{1}, \psi_{1}\right)=\sup _{\psi_{2} \in \mathscr{S}} \frac{1}{4} \frac{\Omega\left(\psi_{1}, \psi_{2}\right)^{2}}{\mu\left(\psi_{2}, \psi_{2}\right)},
$$

onde sup denota o supremo, i.e., o menor real que é maior ou igual aos possíveis valores da razão acima. Uma vez de posse de $\mu$, defina o espaço de Hilbert real $\mathscr{S}_{\mu}$ através do completamento de $\mathscr{S}$ pela norma definida por $\mu$. Em poucas palavras, o procedimento de completamento consiste em formar um conjunto, aqui denotado por $\mathscr{S}_{\mu}$, ao qual pertencem todos os elementos de $\mathscr{S}$ e o limite de todas as seqüências de Cauchy — segundo a norma definida por $\mu$ - que podem ser formadas com elementos de $\mathscr{S}$ (para mais detalhes, veja, e.g., a referência (32)). Por continuidade, então, é possível estender $\mu$ e $\Omega$ a $\mathscr{S}_{\mu}$ e, assim, definir o produto interno de $\mathscr{S}_{\mu} \operatorname{como}\langle\cdot, \cdot\rangle_{\mathscr{S}_{\mu}}:=2 \mu(\cdot, \cdot)$, onde o fator 2 é colocado por conveniência. Note que se fixado $\psi \in \mathscr{S}_{\mu}, \Omega(\cdot, \psi): \mathscr{S}_{\mu} \rightarrow \mathbb{R}$ define um funcional linear e, por conta da Eq. (3.1.13), limitado, $l_{\psi}(\sigma):=\Omega(\sigma, \psi)$. Pelo Teorema da Representação de Reiz (veja, e.g., o Teorema 31.9 da referência (32)), existe, então, um único vetor $\chi \in \mathscr{S}_{\mu}$ tal que $\langle\sigma, \chi\rangle_{\mathscr{S}_{\mu}}=\Omega(\sigma, \psi)$. Isso implica que $\Omega$ define $\hat{J}: \mathscr{S}_{\mu} \rightarrow \mathscr{S}_{\mu}$, ${ }^{3}$ Se $u, v \in \mathscr{H}$, então $u \otimes_{\mathrm{s}} v:=\frac{u \otimes v+v \otimes u}{2}$. 
um operador limitado, tal que

$$
\langle\sigma, \hat{J} \psi\rangle_{\mathscr{S}_{\mu}}=\Omega(\sigma, \psi)
$$

Segue da Eq. (3.1.14 que $\hat{J}^{\dagger}=-\hat{J}$ e $\hat{J}^{2}=-\hat{1}$; por conta dessas propriedades, $\hat{J}$ é chamado de forma complexa. O passo seguinte consiste na complexificação de $\mathscr{S}$ a fim de se obter o espaço de Hilbert complexo $\mathscr{S}_{\mu}^{\mathbb{C}}$ e a extensão a este, por linearidade, de $\mu, \Omega$ e $\hat{J}$, extensões estas também denotadas, respectivamente, por $\mu, \Omega$ e $\hat{J}$. Feito isso, defini-se sobre $\mathscr{S}_{\mu}^{\mathbb{C}}$ o produto interno $\langle\cdot, \cdot\rangle_{\mathscr{S}_{\mu}^{\mathbb{C}}}:=2 \mu(\cdot, \cdot)$, onde a barra na primeira entrada da extensão a $\mathscr{S}_{\mu}^{\mathbb{C}}$ - de $\mu$ denota o complexo conjugado. Em $\mathscr{S}_{\mu}^{\mathbb{C}}$, îे é um operador limitado auto-adjunto o qual, por meio Teorema Espectral (veja, e.g., o Teorema 32.38 da referência (32)), pode ser escrito como

$$
i \hat{J}=\hat{P}_{+}-\hat{P}_{-} .
$$

Os operadores $\hat{P}_{ \pm}$são os projetores espectrais de $i \hat{J}$ e são responsáveis por projetar os vetores de $\mathscr{S}_{\mu}^{\mathbb{C}}$, respectivamente, nas partes positiva e negativa do espectro de $i \hat{J}$. Por fim, defini-se o espaço de Hilbert $\mathscr{H} \subset \mathscr{S}_{\mu}^{\mathbb{C}}$ como sendo a imagem de $\mathscr{S}_{\mu}^{\mathbb{C}}$ pelo projetor $\hat{P}_{-}$. Note que, para quaisquer dois vetores de $\mathscr{H}$,

$$
\begin{aligned}
\left\langle\hat{P}_{ \pm} \psi_{1}, \hat{P}_{ \pm} \psi_{2}\right\rangle_{\mathscr{S}_{\mu}^{\mathbb{C}}} & =\Omega\left(\overline{\hat{P}_{ \pm} \psi_{1}}, \hat{J}^{-1} \hat{P}_{ \pm} \psi_{2}\right) \\
& =-\Omega\left(\overline{\hat{P}_{ \pm} \psi_{1}}, \hat{J} \hat{P}_{ \pm} \psi_{2}\right) \\
& = \pm i \Omega\left(\overline{\hat{P}_{ \pm} \psi_{1}}, \hat{P}_{ \pm} \psi_{2}\right)
\end{aligned}
$$

que é positivo quando $\psi_{1}=\psi_{2} \neq 0$. Além disso,

$$
\left\langle\overline{\hat{P}_{-} \psi}, \hat{P}_{-} \psi\right\rangle_{\mathscr{S}_{\mu}^{\mathbb{C}}}=-i \Omega\left(\hat{P}_{-} \psi, \hat{P}_{-} \psi\right)=0
$$

por conta da anti-simetria da forma simplética, o que significa que $\left(\hat{P}_{-} \psi\right)^{\perp}$, o vetor ortogonal a $\hat{P}_{-} \psi$, satisfaz

$$
\left(\hat{P}_{-} \psi\right)^{\perp}=\overline{\hat{P}_{-} \psi}
$$

Do resultado da Eq. (3.1.16) ainda decorre que

$$
\left.\overline{\left\langle\hat{P}_{-} \psi_{1}\right.}, \overline{\hat{P}_{-} \psi_{2}}\right\rangle_{\mathscr{S}_{\mu}^{\mathbb{C}}}=-\left\langle\hat{P}_{-} \psi_{1}, \hat{P}_{-} \psi_{2}\right\rangle_{\mathscr{S}_{\mu}^{\mathbb{C}}}
$$


No caso particular em que $\psi_{1}, \psi_{2} \in \mathscr{S}$, ainda é possível mostrar que

$$
\left\langle\hat{P}_{-} \psi_{1}, \hat{P}_{-} \psi_{2}\right\rangle_{\mathscr{S}_{\mu}^{\mathbb{C}}}=\mu\left(\psi_{1}, \psi_{2}\right)-\frac{i}{2} \Omega\left(\psi_{1}, \psi_{2}\right)
$$

Defini-se, então, o produto interno de $\mathscr{H}$, ou produto interno de $\mathrm{KG},(\cdot, \cdot)_{\mathrm{KG}}: \mathscr{H} \times \mathscr{H} \rightarrow$ $\mathbb{C}$, comq $^{4}$

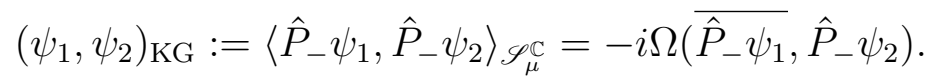

Neste ponto fica claro que o papel de $\mu$ é prover uma forma de dividir $\mathscr{S}_{\mu}^{\mathbb{C}}$ em dois subespaços ortogonais, $\mathscr{H}$ e $\mathscr{H}^{\perp}$, este último sendo a imagem de $\mathscr{S}_{\mu}^{\mathbb{C}}$ pelo projetor $\hat{P}_{+}$. O resultado na Eq. 3.1.18 implica que há uma relação unívoca entre o complexo conjugado dos vetores de $\mathscr{H}$ e os vetores do espaço ortogonal a $\mathscr{H}$. Isso faz com que seja possível identificar $\overline{\mathscr{H}}$, o espaço complexo conjugado a $\mathscr{H}$, com $\mathscr{H}^{\perp}$ e, assim, escrever $\mathscr{S}_{\mu}^{\mathbb{C}}=\mathscr{H} \oplus \overline{\mathscr{H}}:^{5}$

De posse do espaço de Hilbert $\mathscr{H}$ é possível então prosseguir com a construção da representação do comutador dado na Eq. 3.1.9 e, conseqüentemente, das relações de Weyl Eq. (3.1.8). Assim, dado $\Psi \in \mathscr{F}_{\mathrm{s}}(\mathscr{H})$, denotado por

$$
\Psi:=\left(c, \psi^{(1)}, \psi^{(2)}, \psi^{(3)}, \ldots\right)
$$

defina para $\sigma, \tau \in \mathscr{S}_{\mu}^{\mathbb{C}}$ os operadores

$$
\hat{a}\left(\overline{\hat{P}_{-} \sigma}\right) \Psi:=\left(\left(\hat{P}_{-} \sigma, \psi^{(1)}\right)_{\mathrm{KG}}, \sqrt{2} \hat{P}_{-} \sigma \cdot \psi^{(2)}, \sqrt{3} \hat{P}_{-} \sigma \cdot \psi^{(3)}, \ldots\right),
$$

chamado de operador de destruição, e

$$
\hat{a}^{\dagger}\left(\hat{P}_{-} \tau\right) \Psi:=\left(0, c \hat{P}_{-} \tau, \sqrt{2} \hat{P}_{-} \tau \otimes_{\mathrm{s}} \psi^{(1)}, \sqrt{3} \hat{P}_{-} \sigma \otimes_{\mathrm{s}} \psi^{(2)}, \ldots\right)
$$

seu hermitiano conjugado, o operador de criação. Aqui $\hat{P}_{-} \sigma \cdot \psi^{(n)}$ denota o produto de KG entre $\hat{P}_{-} \sigma$ e o primeiro $\mathscr{H}$ que aparece em cada parcela do produto tensorial simetrizado,

\footnotetext{
${ }^{4}$ Para ser consistente com a notação empregada ao longo desta seção, o produto interno de $\mathscr{H}$ deveria ser denotado por $\langle\cdot, \cdot\rangle_{\mathscr{H}}$. Entretanto, optei por adotar a notação mais comum na literatura.

${ }^{5} \mathrm{O}$ espaço vetorial complexo $V$ e seu complexo conjugado $\bar{V}$ consistem no mesmo conjunto de elementos, no entanto a multiplicação de um elemento de $\bar{V}$ por um escalar $c$ retorna o mesmo vetor que seria obtido se tivéssemos multiplicado o mesmo elemento de $V$ por $\bar{c}$.
} 
i.e., para $\psi^{(2)}=\psi_{1} \otimes_{\mathrm{s}} \psi_{2}$, por exemplo,

$$
\hat{P}_{-} \sigma \cdot \psi^{(2)}=\frac{\left(\hat{P}_{-} \sigma, \psi_{1}\right)_{\mathrm{KG}} \psi_{2}+\left(\hat{P}_{-} \sigma, \psi_{2}\right)_{\mathrm{KG}} \psi_{1}}{2} .
$$

Por conta da forma com que foram definidos, os operadores de criação e destruição satisfazem

$$
\begin{gathered}
{\left[\hat{a}\left(\overline{\hat{P}_{-} \sigma}\right), \hat{a}\left(\hat{P}_{-} \tau\right)\right]=0,} \\
{\left[\hat{a}^{\dagger}\left(\hat{P}_{-} \sigma\right), \hat{a}^{\dagger}\left(\hat{P}_{-} \tau\right)\right]=0,}
\end{gathered}
$$

$\mathrm{e}$

$$
\left[\hat{a}\left(\overline{\hat{P}_{-} \sigma}\right), \hat{a}^{\dagger}\left(\hat{P}_{-} \tau\right)\right]=(\sigma, \tau)_{\mathrm{KG}} \hat{1}
$$

Note que tanto em $\hat{W}(\psi)$ quanto em $\hat{\Omega}(\psi, \cdot), \psi \in \mathscr{S}$. Logo, o que nos interessa aqui é o projetor $\hat{P}_{-}$como um mapeamento entre $\mathscr{S}$ e $\mathscr{H}$, ou seja, sua restrição ao espaço formado pelas soluções reais do campo. Vou denotar por $K: \mathscr{S} \rightarrow \mathscr{H}$ tal restrição. Definido dessa forma, a imagem de $K$ é densa em $\mathscr{H}$. Por fim, escrevendo para todo $\psi \in \mathscr{S}$

$$
\hat{\Omega}(\psi, \cdot)=i \hat{a}(\overline{K \psi})-i \hat{a}^{\dagger}(K \psi)
$$

segue, ao serem usadas as relações de comutação da Eq. (3.1.26), a relação de comutação da Eq. 3.1.9.

Já mencionei que existe uma relação entre $\hat{\Omega}(\psi, \cdot)$ e o operador campo usual $\hat{\phi}$. Vejamos como, dentro da construção exposta nos últimos parágrafos, surge tal relação. Para tanto, considere a equação de KG com fonte,

$$
-\nabla_{a} \nabla^{a} \phi+\left(m^{2}+\xi R\right) \phi=f
$$

onde $f \in C_{0}^{\infty}(\mathcal{M})$, o conjunto das funções suaves e de suporte compacto sobre a variedade M. As soluções da Eq. 3.1.28 podem ser escritas como a soma da solução da equação homogênea mais a solução particular, que pode ser a solução particular avançada, $A f$, ou a retarda, $R f$. Uma vez que ambas satisfazem a equação de campo com fonte e que a equação em questão é linear, vem que $E f:=A f-R f$ é solução da equação homogênea 
com condições iniciais de suporte compacto em alguma superfície de Cauchy. Portanto, $E$ pode ser visto como um mapeamento entre o espaço das funções de suporte compacto em $\mathcal{M}$ e o espaço de soluções $\mathscr{S}$. É possível mostrar para esse mapeamento (veja, e.g., o Teorema 3.2.1 da referência (7)) que

(a) para todo $\psi \in \mathscr{S}$ existe $f \in C_{0}^{\infty}(\mathcal{M})$ tal que $\left.\psi=E f\right]^{6}$

(b) $E f=0$ se, e somente se, $f=\nabla_{a} \nabla^{a} g-\left(m^{2}+\xi R\right) g$, com $g \in C_{0}^{\infty}(\mathcal{M})$,

(c) e que para $\psi \in \mathscr{S}$ e $f \in C_{0}^{\infty}(\mathcal{M})$ vale

$$
\Omega(E f, \psi)=\int_{\mathcal{M}} \sqrt{-g} d^{4} x \phi f
$$

Defina, então, o operador $\hat{\Phi}(f)$ como

$$
\hat{\Phi}(f):=\hat{\Omega}(E f, \cdot)=i \hat{a}(\overline{K E f})-i \hat{a}^{\dagger}(K E f) .
$$

Dessa forma, $\hat{\Phi}$ satisfaz a equação de KG Eq. 3.1.2 no sentido de distribuição e se for escrito em termos do operador de Heisenberg $\hat{\phi}$ pesado pela função $f$, então $\hat{\phi}$ satisfaz a equação de campo e as relações de comutação canônicas da Eq. 3.1.11. Ademais, é possível mostrar que, para $f, g \in C_{0}^{\infty}(\mathcal{M})$,

$$
[\hat{\Phi}(f), \hat{\Phi}(g)]=-i \Omega(E f, E g) \hat{1}=-i \int_{\mathcal{M}} \sqrt{-g} d^{4} x f E g \hat{1}
$$

ou, em termos de $\hat{\phi}$, vem que

$$
\left[\hat{\phi}(x), \hat{\phi}\left(x^{\prime}\right)\right]=-i E\left(x, x^{\prime}\right) \hat{1}
$$

onde $E\left(x, x^{\prime}\right)$ denota a diferença entre as funções de Green avançada e retarda da Eq. 3.1.2 e é conhecido na literatura como função de Pauli-Jordan ou de Schwinger (5). Por fim, para o estado de vácuo da representação, o vetor normalizado

$$
\Psi_{0}=(c, 0,0,0, \ldots)
$$

\footnotetext{
${ }^{6}$ Para ser coerente com a notação adotada ao longo desta seção, seria mais preciso escrever $\psi=$ $\left(E f, \sqrt{h} n^{a} \nabla_{a} E f\right)$. Todavia, usarei a notação $E f$ tanto para denotar o campo quanto para o correspondente elemento de $\mathscr{S}$.
} 
tem-se que

$$
\begin{aligned}
\langle 0|\hat{\Phi}(f) \hat{\Phi}(g)| 0\rangle & :=\left\langle\Psi_{0}, \hat{\Phi}(f) \hat{\Phi}(g) \Psi_{0}\right\rangle_{\mathscr{F}_{\mathrm{s}}(\mathscr{H})} \\
& =\mu(E f, E g)-\frac{i}{2} \int_{\mathcal{M}} \sqrt{-g} d^{4} x f E g
\end{aligned}
$$

onde a igualdade pode ser obtida utilizando-se a propriedade da Eq. 3.1.20). O resultado da Eq. (3.1.34 mostra que o produto interno $\mu$ assumido para $\mathscr{S}$ guarda relação com a parte real da chama função de dois pontos associada ao campo quântico.

Normalmente a quantização do campo é feita numa linha diferente da apresentada aqui. Usualmente, ela é implementada escolhendo-se um "conjunto completo de soluções" da Eq. (3.1.2) e decompondo-se o operador campo em termos destas. Vejamos como essa forma de escrever o operador campo, talvez mais familiar do que a definição dada na Eq. 3.1.30, emerge. Para tanto, considere uma base ortonormal completa de $\mathscr{H},\left\{\varphi_{j}\right\}_{j \in I}$. Então, para $\psi \in \mathscr{S}$ tem-se que

$$
K \psi=\sum_{j \in I}\left(\varphi_{j}, K \psi\right)_{\mathrm{KG}} \varphi_{j}
$$

de sorte que, por conta da definição da Eq. (3.1.23,

$$
\hat{a}(\overline{K \psi})=\sum_{j \in I}\left(K \psi, \varphi_{j}\right)_{\mathrm{KG}} \hat{a}_{j}
$$

onde $\hat{a}_{j}:=\hat{a}\left(\overline{\hat{P}_{-} \varphi_{j}}\right)=\hat{a}\left(\bar{\varphi}_{j}\right)$. Agora, escolhendo $f \in C_{0}^{\infty}(\mathcal{M})$ de modo que $\psi=E f$ e usando a definição do produto interno de KG Eq. (3.1.21) e a propriedade dada na Eq. 3.1 .29 , segue que

$$
\hat{a}(\overline{K \psi})=-i \sum_{j \in I} \int_{\mathcal{M}} \sqrt{-g} d^{4} x f \varphi_{j} \hat{a}_{j}
$$

O mesmo caminho para o operador de criação leva a

$$
\hat{a}^{\dagger}(K \psi)=i \sum_{j \in I} \int_{\mathcal{M}} \sqrt{-g} d^{4} x f \bar{\varphi}_{j} \hat{a}_{j}^{\dagger}
$$

onde $\hat{a}_{j}^{\dagger}:=\hat{a}^{\dagger}\left(\hat{P}_{-} \varphi_{j}\right)=\hat{a}^{\dagger}\left(\varphi_{j}\right)$. Por fim, a substituição da Eq. 3.1.37 e da Eq. 3.1.38 na definição da Eq. 3.1.30 leva à forma mais usual de se escrever $\hat{\Phi}$ :

$$
\hat{\Phi}(f)=\sum_{j \in I} \int_{\mathcal{M}} \sqrt{-g} d^{4} x f\left(\varphi_{j} \hat{a}_{j}+\bar{\varphi}_{j} \hat{a}_{j}^{\dagger}\right)
$$


Essa é uma forma interessante de se escrever o operador campo pois sugere que cada "grau de liberdade" $j$ do campo, "descrito" pelo vetor $\varphi_{j}$, se comporta como um oscilador harmônico quântico. De fato, haja visto que, por conta da Eq. (3.1.26), segue que

$$
\begin{aligned}
& {\left[\hat{a}_{j}, \hat{a}_{k}\right]=0,} \\
& {\left[\hat{a}_{j}^{\dagger}, \hat{a}_{k}^{\dagger}\right]=0,}
\end{aligned}
$$

e que

$$
\left[\hat{a}_{j}, \hat{a}_{k}^{\dagger}\right]=\delta_{j k} \hat{1}
$$

A base $\left\{\varphi_{j}\right\}_{j \in I}$ escolhida para $\mathscr{H}$, entretanto, é arbitrária e, portanto, sua escolha não carrega, em princípio, nenhuma física. Dito de outro modo, o conjunto de vetores $\left\{\varphi_{j}\right\}_{j \in I}$ está para $\mathscr{H}$ assim como um sistema de coordenadas está para o espaço-tempo na RG.

As motivações por detrás da construção apresentada nos últimos parágrafos são as seguintes (34). Em primeiro lugar, a construção da representação das relações de Weyl Eq. 3.1.8 como um espaço de Fock sobre um espaço de Hilbert $\mathscr{H}$ reflete a visão de que campos quânticos descrevem um conjunto de partículas: um elemento de $\mathscr{H}$ representaria o estado quântico de uma partícula enquanto que um elemento de $\mathscr{F}_{\mathrm{s}}(\mathscr{H})$ descreveria algum estado de superposição de $N$ partículas, para diferentes valores de $N$. Em segundo lugar, a definição de $\mathscr{H}$ a partir do espaço de soluções da Eq. (3.1.2) está ligada à noção de que na Mecânica Quântica relativística de um partícula as soluções complexas da Eq. (3.1.2) seriam funções de onda. Em último lugar, a definição do operador campo em termos de operadores de criação e destruição reflete a idéia intuitiva de que um campo obedecendo uma equação de onda pode ser visto como uma coleção de osciladores harmônicos. Entretanto, ao menos no que tange ao conceito de partículas, essas motivações têm simplesmente um caráter heurístico, não sendo, portanto, a finalidade da construção acima. De fato, ao contrário do que pode parecer quando, por exemplo, é estudada a quantização do campo eletromagnético em espaço plano e sua interação com a matéria, o conceito de partícula somente emerge a posteriori, através da interpretação de certos 
processos e não como elemento fundamental da teoria.7

Note que até este ponto o produto interno $\mu$, peça-chave na construção apresentada acima, ainda não foi definido. 8 A questão que se coloca, então, é a da existência desse produto interno e, se existir, sua unicidade. Em espaços-tempos globalmente hiperbólicos gerais é possível mostrar que sempre existe um $\mu$ satisfazendo a condição dada na Eq. 3.1.13 (36), entretanto não se conhece — e acredita-se não haver (7) — um critério, em geral, para privilegiar uma entre as diferentes formas possíveis para $\mu$. Pior do que isso, duas escolhas diferentes para $\mu$ podem levar a representações unitariamente nãoequivalentes para as relações de Weyl dadas na Eq. (3.1.8), ou seja, em princípio cada representação pode descrever uma realidade física diferente. A despeito disso, foi mostrado na referência (37), dentro do formalismo algébrico para campos quânticos (38), que a álgebra definida pelas relações de Weyl independe da escolha feita para a representação. No que tange ao problema tratado nesta tese, a linha principal de argumentação passará ao largo dessas questões uma vez que darei atenção a espaços-tempos assintoticamente estáticos. Apesar de não haver uma prescrição de quantização para campos privilegiada em espaços-tempos gerais, para aqueles espaços-tempos que possuem uma isometria temporal, mesmo que assintótica, existe uma escolha natural para o produto interno $\mu$.

\subsection{Quantização em espaços-tempos estacionários}

Para mostrar de que maneira a escolha de $\mu$ é feita no caso estacionário faz-se mister, em primeiro lugar, definir o tensor energia-momentum associado ao campo $\phi$. Dada a ação $S[\phi]$ que define o campo clássico, defini-se o tensor energia-momentum $T_{a b}$ associado a

\footnotetext{
${ }^{7}$ No caso particular do espaço-tempo de Minkowski, E. Wigner define o conceito de partícula usando as representações irredutíveis do grupo de Poincaré (35). Essa visão do conceito de partícula será discutida brevemente no Capítulo 8.

${ }^{8} \mathrm{~A}$ construção acima poderia ter sido feita dando uma forma complexa $\hat{J}$ sobre $\mathscr{S}$ e, através dela, definindo $\mu$. A estratégia de se definir uma forma complexa em $\mathscr{S}$ para quantizar o campo é adotada, por exemplo, por Ashtekar e Magnon na referência (34).
} 
esse campo por

$$
\left.T_{a b}:=-\frac{2}{\sqrt{-g}} \frac{\delta S[\phi]}{\delta g^{a b}}, 9\right]
$$

Para a ação da Eq. (3.1.1) essa definição implica no seguinte tensor simétrico:

$$
\begin{aligned}
T_{a b}= & (1-2 \xi) \nabla_{a} \phi \nabla_{b} \phi+\xi R_{a b} \phi^{2}-2 \xi \phi \nabla_{a} \nabla_{b} \phi \\
& +\frac{4 \xi-1}{2}\left[\nabla_{c} \phi \nabla^{c} \phi+\left(m^{2}+\xi R\right) \phi^{2}\right] g_{a b} .
\end{aligned}
$$

O tensor energia-momentum associado ao campo é um observável importante pois nos provê informações locais sobre energia, momentum e estresse do campo. Além disso, essa é uma quantidade central se estamos preocupados com o papel gravitacional que o campo pode desempenhar, uma vez que $T_{a b}$ aparece como fonte da equação de Einstein Eq. 2.1.5). Em particular, se $\phi$ satisfaz a equação de campo Eq. (3.1.2), então é possível mostrar que

$$
\nabla_{a} T_{b}^{a}=0
$$

Considere um observador com quadrivelocidade $u^{a}$. O escalar $u^{a} u^{b} T_{a b}$ tem a interpretação de ser a densidade de energia atribuída pelo observador ao campo escalar no instante definido pela superfície de Cauchy que passa pelo ponto em questão. Quando o espaçotempo possui uma isometria temporal gerada pelo campo de Killing $\varkappa^{a}$, ao se contrair os dois lados da Eq. (3.2.3) com $\varkappa^{a}$ e integrá-los numa porção $\mathcal{N} \subset \mathcal{M}$ do espaço-tempo definida por ter como fronteira duas superfícies de Cauchy, $\Sigma_{t_{1}}$ e $\Sigma_{t_{2}}$, com $t_{2}>t_{1}$, segue que

$$
\int_{\Sigma_{t_{1}}} d \Sigma n^{a} \varkappa^{b} T_{a b}=\int_{\Sigma_{t_{2}}} d \Sigma n^{a} \varkappa^{b} T_{a b}
$$

onde $d \Sigma=\sqrt{h} d^{3} x$ denota o elemento de volume da hiperfície $\Sigma_{t}$. Para derivar o resultado acima basta integrar por partes e utilizar o Teorema de Stokes (veja, e.g., o Teorema B.2.1 da referência (4)). Esse resultado implica que para uma família de observadores seguindo as linhas integrais do campo $\varkappa^{a}$ a energia total associada ao campo é conservada.

Seja $(\mathcal{M}, g)$ um espaço-tempo globalmente hiperbólico estacionário e $\varkappa^{a}$ o campo

${ }^{9}$ Para uma discussão sobre a definição do tensor energia-momentum em teoria de campos, veja, e.g., as referências $(39,40)$. 
vetorial que gera a isometria temporal $\alpha_{t}: \mathcal{M} \rightarrow \mathcal{M}$. A construção de $\mu$ em espaçostempos estacionários pode ser feita da seguinte forma $(7,34,41)$. Considere o espaço $\mathscr{S}^{\mathbb{C}}$, obtido através da complexificação do espaço de soluções $\mathscr{S}$. Defina sobre esse espaço de soluções complexas o produto internd $\alpha^{10}\langle\cdot, \cdot\rangle: \mathscr{S}^{\mathbb{C}} \times \mathscr{S}^{\mathbb{C}} \rightarrow \mathbb{C}$ de sorte que

$$
\left\langle\psi_{1}, \psi_{2}\right\rangle:=\int_{\Sigma_{t}} d \Sigma n^{a} \varkappa^{b} T_{a b}\left(\psi_{1}, \psi_{2}\right)
$$

onde

$$
\begin{aligned}
T_{a b}\left(\psi_{1}, \psi_{2}\right):= & (1-2 \xi) \nabla_{(a} \bar{\phi}_{1} \nabla_{b)} \phi_{2}+\xi R_{a b} \bar{\phi}_{1} \phi_{2}-\xi\left(\bar{\phi}_{1} \nabla_{a} \nabla_{b} \phi_{2}+\phi_{2} \nabla_{a} \nabla_{b} \bar{\phi}_{1}\right) \\
& +\frac{4 \xi-1}{2}\left[\nabla_{c} \bar{\phi}_{1} \nabla^{c} \phi_{2}+\left(m^{2}+\xi R\right) \bar{\phi}_{1} \phi_{2}\right] g_{a b} .
\end{aligned}
$$

Uma vez que $\phi_{1}$ e $\phi_{2}$ são soluções complexas da equação de KG Eq. (3.1.2), tem-se que o lado direito da Eq. (3.2.5) é conservado e assim é independe da superfície de Cauchy sobre a qual a integral é calculada. Sobre $\mathscr{S}^{\mathbb{C}}$ é também possível definir o mapa de translação temporal $\tau_{t}: \mathscr{S}^{\mathbb{C}} \rightarrow \mathscr{S}^{\mathbb{C}}$ tal que

$$
\tau_{t}(\psi)=\left(\phi \circ \alpha_{-t}, \pi \circ \alpha_{-t}\right)
$$

De fato, já que, por conta da simetria do espaço-tempo de fundo, se $\phi$ é solução da Eq. 3.1.2), então sua composição com a isometria $\alpha_{t}$ também é solução. A ação do mapa $\tau_{t}$ consiste em pegar uma solução $\psi$ com condições iniciais, digamos, na hiperfície $\Sigma_{t_{0}}$ e levá-la na solução $\tau_{t}(\psi)$, que tem exatamente as mesmas condições iniciais que $\psi$ mas na hiperfície $\Sigma_{t_{0}-t}$. Por conta da definição de isometria, dada na Seção 2.1, e da definição dada na Eq. (3.2.7) segue que $\tau_{t_{1}} \circ \tau_{t_{2}}=\tau_{t_{1}+t_{2}}$, o que implica que a isometria $\alpha_{t}$ define um grupo em $\mathscr{S}^{\mathbb{C}}$. Ademais, é possível mostrar que

$$
\left\langle\tau_{t}\left(\psi_{1}\right), \tau_{t}\left(\psi_{2}\right)\right\rangle=\left\langle\psi_{1}, \psi_{2}\right\rangle
$$

de modo que, do ponto de vista do produto interno da Eq. 3.2.5), $\tau_{t}$ é uma transformação de simetria de $\mathscr{S}^{\mathbb{C}}$.

\footnotetext{
${ }^{10}$ Para espaços-tempos de fundo e valores de $\xi$ arbitrários, a forma dada pelo tensor energia-momentum pode não ser positiva quando $\psi_{1}=\psi_{2}$. Entretanto, é possível mostrar em casos particulares, como quando a geometria de fundo é plana ou quando o campo tem acoplamento mínimo, que essa forma de fato define um produto interno em $\mathscr{S}^{\mathbb{C}}$.
} 
Defina, então, o espaço de Hilbert $\mathscr{E}$ através do completamento de $\mathscr{S}^{\mathbb{C}}$ na norma definida pelo produto interno dado na Eq. 3.2.5. Em $\mathscr{S}^{\mathbb{C}}, \tau_{t}$ é linear, por conta de sua definição da Eq. 3.2.7), e é limitado, por conta da propriedade Eq. 3.2.8. Uma vez que, por construção, o domínio de $\tau_{t}$ é denso em $\mathscr{E}$, segue pelo Teorema BLT (veja, e.g., o Teorema 32.1 da referência $(32)$ ) que existe uma única extensão de $\tau_{t}$ a $\mathscr{E}, \hat{V}_{t}$, que é linear e limitada e cuja norma é igual a de $\tau_{t}$ em $\mathscr{S}^{\mathbb{C}}$. Como a propriedade Eq. 3.2.8 também é satisfeita quando $\tau_{t}$ é substituído por $\hat{V}_{t}$ e $\alpha_{t}$ é um mapa $C^{\infty}$, então $\hat{V}_{t}$ forma um grupo unitário a um parâmetro fortemente contínuo atuando em $\mathscr{E}$. Pelo Teorema de Stone, então, existe um operador auto-adjunto $\hat{h}$ tal que $\hat{V}_{t}=e^{-i t \hat{h}}$. Como $\hat{h}$ satisfaz (veja, e.g., o Teorema VIII.7 da referência $(30))$

$$
\lim _{t \rightarrow 0} \frac{\hat{V}_{t} \psi-\psi}{t}=-i \hat{h} \psi
$$

para $\psi \in \mathscr{S}^{\mathbb{C}}$, então, pela definição da derivada de Lie Eq. 2.1.7), segue que

$$
\hat{h} \psi=\left(i £_{\varkappa} \phi, i £_{\varkappa} \pi\right)
$$

Note ainda que o domínio de $\hat{h}$ contem o espaço $\mathscr{S}^{\mathbb{C}}$, pois como os elementos de $\mathscr{S}^{\mathbb{C}}$ são pares de funções suaves se a variedade é suave (ver, e.g., Teorema 2.1 de (41)), então suas derivadas temporais são finitas e suaves. Logo, a imagem de $\hat{h}$ também contem $\mathscr{S}^{\mathbb{C}}$. Uma vez que $£_{\varkappa} \psi=0$ para $\psi$ com suporte compacto em qualquer hiperfície $\Sigma_{t}$ se, e somente se, $\psi=0$, então $\hat{h}$ é bijetor e existe $\hat{h}^{-1}$.

Suponha agora que $m>0$ e que existe uma superfície de Cauchy sobre a qual $-\varkappa^{a} \varkappa_{a} \geq-\varepsilon \varkappa^{a} n_{a}>\varepsilon^{2}$ para algum $\varepsilon>0$. Nesse caso é possível mostrar que existe uma constante $C(\varepsilon, m)>0$ tal que a forma simplética $\Omega$, para $\psi_{1}, \psi_{2} \in \mathscr{S}^{\mathbb{C}}$, satisfaz a seguinte desigualdade ${ }^{11}$.

$$
\left|\Omega\left(\bar{\psi}_{1}, \psi_{2}\right)\right|^{2} \leq C(\varepsilon, m)\left\langle\psi_{1}, \psi_{1}\right\rangle\left\langle\psi_{2}, \psi_{2}\right\rangle
$$

Assim, como $\Omega$ é uma forma bilinear e limitada na norma definida por $\langle\cdot, \cdot\rangle$, segue que ${ }^{11}$ Para a prova dessa desigualdade, veja a referência $(41)$. 
é possível estendê-la univocamente para todo $\mathscr{E}$. Além disso, é possível mostrar que

$$
\Omega\left(\bar{\psi}_{1}, \hat{h} \psi_{2}\right)=2 i\left\langle\psi_{1}, \psi_{2}\right\rangle
$$

A propriedade Eq. 3.2.12 e a desigualdade dada na Eq. 3.2.11 combinadas implicam que $\hat{h}^{-1}$ é um operador limitado e que, portanto, zero não pertence ao espectro de $\hat{h}$, $\sigma(\hat{h})$. Aplicando o Teorema Espectral ao operador $\hat{h}$ é possível definir $\hat{E}^{+}: \mathscr{E} \rightarrow \mathscr{E}$, o projetor associado à parte positiva do espectro de $\hat{h}$. Finalmente, dado o projetor $\hat{E}^{+}$, defina $\mu: \mathscr{S} \times \mathscr{S} \rightarrow \mathbb{R}$ como

$$
\mu\left(\psi_{1}, \psi_{2}\right):=\operatorname{Im} \Omega\left(\hat{E}^{+} \psi_{1}, \hat{E}^{+} \psi_{2}\right)=2 \operatorname{Re}\left\langle\hat{E}^{+} \psi_{1}, \hat{h}^{-1} \hat{E}^{+} \psi_{2}\right\rangle
$$

Definido dessa forma $\mu$ é um produto interno sobre $\mathscr{S}$ e satisfaz a condição da Eq. 3.1.13. ${ }^{12}$ Uma vez de posse de $\mu$, pode-se, então, implementar a construção exposta na Seção 3.1. O espaço de Hilbert $\mathscr{H}$, entretanto, pode ser obtido sem mais delongas já neste ponto ao notar-se que a definição da Eq. 3.2.13) é consistente com a forma complexa $\hat{J}=i \hat{h}|\hat{h}|^{-1}$, onde $|\hat{h}|:=\sqrt{\hat{h}^{\dagger} \hat{h}}$. Desse modo, $\hat{P}_{-}=\hat{E}^{+}$e $\mathscr{H}$ é a imagem de $\mathscr{E}$ por $\hat{E}^{+}$ completada através do produto interno de KG engendrado pelo projetor $\hat{E}^{+}$.

A idéia por detrás dessa construção é fazer com que os "estados quânticos de uma partícula", i.e., os vetores de $\mathscr{H}$, possam ser escritos em termos de combinações lineares de "auto-estados de energia positiva". De fato, se a coordenada temporal $t$ escolhida é aquela definida pela isometria, pode-se escrever a Eq. 3.2.10 como

$$
\hat{h} \psi=\left(i \partial_{t} \phi, i \partial_{t} \pi\right)
$$

Uma vez que em Mecânica Quântica o operador $i \partial_{t}$ tem a interpretação de ser o operador energia, o espectro de $\hat{h}$ codifica os possíveis valores para essa grandeza que - do ponto de vista dos observadores seguindo as linhas integrais de $\varkappa^{a}$ — podem ser obtidos numa medida. Portanto, numa medida da energia de um sistema cujo estado é um vetor de $\mathscr{H}$, os possíveis valores que podem retornar são, por construção, estritamente positivos. Sendo assim, a prescrição para a definição de $\mu$ (ou de uma forma complexa) em espaços-

\footnotetext{
${ }^{12}$ Para uma prova dessa última afirmação, veja a referência $(36)$.
} 
tempos estacionários é uma versão, em termos mais precisos, da prescrição de separação das soluções da equação de campo Eq. 3.1.2 em uma parte de freqüência positiva e outra de freqüência negativa.

Em espaços-tempos estáticos, base para a maior parte da discussão que será feita nos próximos capítulos, essa mesma idéia pode se fraseada afirmando-se que a função de onda do sistema deve satisfazer a seguinte equação:

$$
i \frac{\partial}{\partial t} \phi=\sqrt{\Delta} \phi
$$

A forma do operador $\Delta$ será dada na Seção 4.2 e para a discussão neste ponto basta dizer que ela envolve derivadas associadas à seção espacial, a massa do campo e o escalar de curvatura. Se $\Delta$ é um operador positivo na norma de $\mathscr{H}$, então sua raiz pode ser definida através do Teorema Espectral. Pela Eq. 3.2.15 fica claro que o espectro do operador $\hat{h}$ está relacionado com a raiz do espectro do operador $\Delta$. De fato, uma vez que

$$
\hat{h}^{2} \phi=-\frac{\partial^{2}}{\partial t^{2}} \phi=\Delta \phi
$$

deve reproduzir, nas coordenadas escolhidas, a equação de campo Eq. (3.1.2). Uma vez que $\Delta$ guarda relação com $\hat{h}$, é possível usar os projetores espectrais de $\Delta-$ quando realizados em termos de funções sobre uma superfície de Cauchy $\Sigma_{t}$ - para escrever os vetores de $\mathscr{H}$ dados por $K \psi=\left(\phi_{+}, \pi_{+}\right)$da seguinte forma:

$$
\phi_{+}(t, \mathbf{x})=\int_{\mathcal{I}} \frac{d \mu(j)}{\sqrt{2 \omega_{j}}} \tilde{\phi}_{+}(j) F_{j}(\mathbf{x}) e^{-i \omega_{j} t}
$$

e $\pi_{+}$pode ser obtido a partir da definição dada na Eq. (3.1.3). Aqui $\mathcal{I}$ denota um conjunto de índices contínuos ou discretos que indexam os elementos do espectro do operador $\Delta$ e $\mu(j)$ denota uma medida sobre esse conjunto, enquanto que $\mathbf{x}$ denota as coordenadas espaciais definidas sobre a hiperfície $\Sigma_{t}$. A função $F_{j}: \Sigma_{t} \rightarrow \mathbb{C}$, dita autofunção do operador $\Delta$, é definida por

$$
\Delta F_{j}=\omega_{j}^{2} F_{j}
$$


onde $\omega_{j}>0$. As autofunções do operador $\Delta$ devem ser escolhidas de sorte que

$$
\int_{\Sigma_{t}} \frac{d \Sigma}{\|\varkappa\|} F_{j}(\mathbf{x}) \bar{F}_{j^{\prime}}(\mathbf{x})=\delta_{\mu}\left(j, j^{\prime}\right)
$$

$\operatorname{com} \delta_{\mu}$ tal que $\int_{\mathcal{I}} d \mu(j) \delta_{\mu}\left(j, j^{\prime}\right)=1$. A Eq. 3.2.19) é usualmente denominada relação de completeza das autofunções de $\Delta$ e reflete a propriedade de que qualquer par de projetores espectrais são ortogonais entre si caso estejam associados a regiões disjuntas do espectro. Já o coeficiente $\tilde{\phi}_{+}$pode ser escrito em termos das condições iniciais que definem $\psi$. Para tanto, note que a solução $\psi \in \mathscr{S}$ pode ser escrita na forma

$$
\phi(t, \mathbf{x})=\int_{\mathcal{I}} \frac{d \mu(j)}{\sqrt{2 \omega_{j}}}\left[\tilde{\phi}_{+}(j) e^{-i \omega_{j}\left(t-t_{0}\right)}+\tilde{\phi}_{-}(j) e^{i \omega_{j}\left(t-t_{0}\right)}\right] F_{j}(\mathbf{x})
$$

$\mathrm{e}$

$$
\pi(t, \mathbf{x})=-i \frac{\sqrt{h}}{\|\varkappa\|} \int_{\mathcal{I}} \frac{d \mu(j)}{\sqrt{2 \omega_{j}}} \omega_{j}\left[\tilde{\phi}_{+}(j) e^{-i \omega_{j}\left(t-t_{0}\right)}-\tilde{\phi}_{-}(j) e^{i \omega_{j}\left(t-t_{0}\right)}\right] F_{j}(\mathbf{x}) .
$$

A relação de completeza Eq. 3.2.19, então, leva, depois de alguma manipulação, a

$$
\tilde{\phi}_{ \pm}(j)=\sqrt{\frac{\omega_{j}}{2}} \int_{\Sigma_{t_{0}}} \frac{d \Sigma}{\|\varkappa\|} \phi_{0}(\mathbf{x}) \bar{F}_{j}(\mathbf{x}) \pm i \frac{1}{\sqrt{2 \omega_{j}}} \int_{\Sigma_{t_{0}}} d^{3} x \pi_{0}(\mathbf{x}) \bar{F}_{j}(\mathbf{x})
$$

Isso posto, é possível, então, expressar o operador campo em termos das autofunções $F_{j}$. De fato, usando a Eq. 3.1.39 para o operador e forma dada na Eq. 3.2.17) para os vetores de $\mathscr{H}$, vem que

$$
\hat{\Phi}(f)=\sum_{k \in I} \int_{\mathcal{M}} \sqrt{-g} d^{4} x f \int_{\mathcal{I}} \frac{d \mu(j)}{\sqrt{2 \omega_{j}}}\left[\tilde{\varphi}_{k}(j) F_{j} e^{-i \omega_{j} t} \hat{a}_{k}+\bar{\varphi}_{k}(j) \bar{F}_{j} e^{i \omega_{j} t} \hat{a}_{k}^{\dagger}\right]
$$

Essa expressão nada mais é do que o conhecido procedimento de "expandir o campo em modos normais" e que no caso de Minkowski reduz-se à "expansão em termos de ondas planas". Ela será útil nos próximos capítulos. As discussões do Capítulo 4 e uma parte substancial das discussões presentes nos Capítulo 5 e 6 podem ser resumidas ao estudo do espectro do operador diferencial $\Delta$ em diferentes geometrias.

Por vezes os autores referem-se na literatura ao espaço de Hilbert $\mathscr{H}$ como "espaço de Hilbert de uma partícula" (7, 34, 41). Como já mencionado ao final da Seção 3.1. o conceito de partícula não é um elemento fundamental na teoria. Entretanto, no caso de espaços-tempos estacionários essa denominação para o espaço de Hilbert $\mathscr{H}$ é justificada 
por processos nos quais o campo interage por um tempo determinado com um outro sistema quântico com estados de energia bem-definidos como, por exemplo, uma partícula quântica confinada em uma caixa ou um átomo livre de outras interações externas. Esse sistema quântico com o qual o campo é posto em contato usualmente recebe na literatura a denominação de detector. Nesse caso, como mostrado, e.g., na Seção 3.3 da referência (7), as transições induzidas em primeira ordem de perturbação entre os estados do campo e entre os estados do detector podem ser interpretadas como a emissão ou a absorção de partículas do campo pelo detector. Nesse sentido, então, em espaços-tempos que possuam uma isometria temporal os vetores de $\mathscr{F}_{\mathrm{s}}(\mathscr{H})$ têm a interpretação natural de descreverem superposições de estados de ocupação de partículas bosônicas.

A discussão sobre o conceito de partícula em TQCEC será revisitada na Seção 8.1 . quando tratarei com mais detalhes da criação de partículas por conta de mudanças da geometria de fundo.

\subsection{Transformações de Bogoliubov}

Considere agora um espaço tempo globalmente hiperbólico $(\mathcal{M}, g)$ que é estacionário no passado. Isso significa que nesse espaço-tempo existe uma superfície de Cauchy $\Sigma_{t}$ cujo passado está relacionado pela isometria $\alpha$ ao passado da superfície de Cauchy $\Sigma_{t^{\prime}}^{\prime}$ de um espaço-tempo globalmente hiperbólico e estacionário $\left(\mathcal{M}^{\prime}, g^{\prime}\right)$. Assim, o espaço de soluções $\mathscr{S}$ da equação de campo Eq. (3.1.2) sobre o espaço-tempo $(\mathcal{M}, g)$ pode ser identificado com o espaço de soluções $\mathscr{S}^{\prime}$ quando o espaço-tempo de fundo é $\left(\mathcal{M}^{\prime}, g^{\prime}\right)$ da seguinte maneira. Considere um elemento de $\mathscr{S}$ com condições iniciais $\left(\phi_{0}, \pi_{0}\right)$ numa superfície de Cauchy $\Sigma_{t_{0}}$ de $(\mathcal{M}, g)$ que esteja no passado da hiperfície $\Sigma_{t}$. Então, é possível associar a solução definida pelo desenvolvimento de Cauchy das condições iniciais $\left(\phi_{0}, \pi_{0}\right)$ à definida pelo desenvolvimento de Cauchy das condições iniciais $\left(\phi_{0} \circ \alpha, \pi_{0} \circ \alpha\right)$ no espaço-tempo $\left(\mathcal{M}^{\prime}, g^{\prime}\right)$. Feita a identificação entre $\mathscr{S}$ e $\mathscr{S}^{\prime}$, é possível induzir um produto interno $\mu \mathrm{em}$ $\mathscr{S}$ através do produto interno $\mu^{\prime}$ definido em $\mathscr{S}^{\prime}$, por exemplo, pelo algoritmo apresentado 
na seção anterior. O espaço de Hilbert definido por essa construção em $\mathscr{S}$ é usualmente denotado por $\mathscr{H}_{\text {in. }}$. Desse modo o espaço de Fock $\mathscr{F}_{\mathrm{s}}\left(\mathscr{H}_{\text {in }}\right)$ tem aquela interpretação de descrever estados de ocupação de partículas no passado da hiperfície $\Sigma_{t}$, mas não no futuro. O mesmo pode ser feito quando $(\mathcal{M}, g)$ é estacionário no futuro. Nesse caso, a mesma receita leva ao espaço de Hilbert $\mathscr{H}_{\text {out }}$, de sorte que $\mathscr{F}_{\mathrm{s}}\left(\mathscr{H}_{\text {out }}\right)$ descreve estados de ocupação de partícula no futuro de $\Sigma_{t}$, mas não no passado. Considerações semelhantes se aplicam mesmo quando o espaço-tempo de fundo é só assintoticamente estacionário no passado ou futuro. É possível mostrar que para espaços-tempos que possuam uma região estacionária no passado e outra no futuro existe um mapeamento linear e unitário, algumas vezes tratado na literatura de TQCEC por matriz $S$, que leva $\mathscr{F}_{\mathrm{s}}\left(\mathscr{H}_{\text {in }}\right)$ em $\mathscr{F}_{\mathrm{s}}\left(\mathscr{H}_{\text {out }}\right)$ e que, conseqüentemente, codifica a criação espontânea de partículas e processos de espalhamento devidos a mudanças na geometria de fundo (veja, e.g., o Teorema 4.4.1 da referência $(7)$ ou a referência $(42)){ }^{13}$

Esse mapeamento, quando implementado no nível do espaço de Hilbert $\mathscr{S}_{\mu}^{\mathbb{C}}$, recebe o nome de transformação de Bogoliubov. Apesar de poderem pertencer a um contexto mais geral, considere, a título de interesses futuros, um espaço-tempo globalmente hiperbólico $(\mathcal{M}, g)$ que é assintoticamente estacionário tanto no passado quanto no futuro. Sejam $\left\{\varphi_{k}^{\text {in }}\right\}_{k \in I}$ uma base ortonormal completa de $\mathscr{H}_{\text {in }}$ e $\left\{\varphi_{l}^{\text {out }}\right\}_{l \in O}$ uma base ortonormal completa de $\mathscr{H}_{\text {out }}$. Com a suposição de que o mesmo espaço de Hilbert formado a partir das soluções complexas da equação de campo Eq. 3.1 .2 pode ser escrito como $\mathscr{H}_{\text {in }} \oplus \overline{\mathscr{H}}_{\text {in }}$ ou $\mathscr{H}_{\text {out }} \oplus \overline{\mathscr{H}}_{\text {out }}$, é possível escrever

$$
\varphi_{k}^{\text {in }}=\sum_{l \in O} A_{k l} \varphi_{l}^{\text {out }}-\bar{B}_{k l} \bar{\varphi}_{l}^{\text {out }}
$$

com $A_{k l}, B_{k l} \in \mathbb{C}$ para todo $k \in I$ e $l \in O$. A partir das relações de ortogonalidade das bases in e out, segue que os coeficientes da combinação linear Eq. 3.3.1 podem ser

\footnotetext{
${ }^{13} \mathrm{O}$ propósito da matriz $S$ não se circunscreve ao cálculo da produção espontânea de partículas ou processos de espalhamento por conta da mudança na geometria de fundo. A discussão sobre a existência desse mapeamento se insere na questão mais ampla de quais são as condições necessárias e suficientes para garantir que duas representações distintas para as relações de Weyl sejam unitariamente equivalentes. Essa questão pode ser traduzida no problema de sob quais condições, dados dois produtos internos sobre $\mathscr{S}$ distintos $\mu$ e $\mu^{\prime}$, satisfazendo a condição dada na Eq. (3.1.13), o completamento de $\mathscr{S}$ na norma de $\mu$ é equivalente ao completamento de $\mathscr{S}$ na norma de $\mu^{\prime}$. Para mais detalhes veja, e.g., Seção 4.4 da referência $(7)$.
} 
escritos em termos do produto interno de KG dado na Eq. 3.1.21 da seguinte forma:

$$
A_{k l}=\left(\varphi_{l}^{\text {out }}, \varphi_{k}^{\text {in }}\right)_{\mathrm{KG}}
$$

e

$$
B_{k l}=-\left(\varphi_{l}^{\text {out }}, \bar{\varphi}_{k}^{\text {in }}\right)_{\mathrm{KG}}
$$

Além disso, a partir da Eq. (3.3.1), é simples mostrar que os coeficientes $A_{k l}$ e $B_{k l}$ têm as seguintes propriedades:

$$
\left(\varphi_{k}^{\text {in }}, \varphi_{k^{\prime}}^{\text {in }}\right)_{\mathrm{KG}}=\sum_{l \in O} \bar{A}_{k l} A_{k^{\prime} l}-B_{k l} \bar{B}_{k^{\prime} l}=\delta_{k k^{\prime}}
$$

e

$$
\left(\bar{\varphi}_{k}^{\mathrm{in}}, \varphi_{k^{\prime}}^{\mathrm{in}}\right)_{\mathrm{KG}}=\sum_{l \in O} A_{k l} \bar{B}_{k^{\prime} l}-A_{k^{\prime} l} \bar{B}_{k l}=0
$$

Já para o operador campo, escrito na forma da Eq. (3.1.39), vem, se usada a Eq. (3.3.1), que

$$
\begin{aligned}
\hat{\Phi}(f) & =\sum_{k \in I} \int_{\mathcal{M}} \sqrt{-g} d^{4} x f\left(\varphi_{k}^{\text {in }} \hat{a}_{k}+\bar{\varphi}_{k}^{\text {in }} \hat{a}_{k}^{\dagger}\right) \\
& =\sum_{k \in I} \int_{\mathcal{M}} \sqrt{-g} d^{4} x f \sum_{l \in O}\left[\varphi_{l}^{\text {out }}\left(A_{k l} \hat{a}_{k}-B_{k l} \hat{a}_{k}^{\dagger}\right)+\bar{\varphi}_{l}^{\text {out }}\left(-\bar{B}_{k l} \hat{a}_{k}+\bar{A}_{k l} \hat{a}_{k}^{\dagger}\right)\right] \\
& =\sum_{l \in O} \int_{\mathcal{M}} \sqrt{-g} d^{4} x f\left(\varphi_{l}^{\text {out }} \hat{b}_{l}+\bar{\varphi}_{l}^{\text {out }} \hat{b}_{l}^{\dagger}\right) .
\end{aligned}
$$

Daí segue a seguinte relação entre os operadores de criação e destruição definidos por cada uma das bases:

$$
\hat{b}_{l}=\sum_{k \in I} A_{k l} \hat{a}_{k}-B_{k l} \hat{a}_{k}^{\dagger}
$$

e

$$
\hat{b}_{l}^{\dagger}=\sum_{k \in I} \bar{A}_{k l} \hat{a}_{k}^{\dagger}-\bar{B}_{k l} \hat{a}_{k}
$$

Às relações dadas na Eq. 3.3.5 dá-se o nome de transformação de Bogoliubov e aos coeficientes $A_{k l}$ e $B_{k l}$ de coeficientes de Bogoliubov.

Considere o caso estático. Escrevamos, então, os vetores $\varphi_{k}^{\text {in }}$ e $\varphi_{l}^{\text {out }}$, respectivamente, em termos dos modos de freqüência positiva no passado e no futuro. Isso significa fazer o seguinte. Tanto no passado quanto no futuro pode-se colocar a equação de campo Eq. 
3.1.2 na forma

$$
-\frac{\partial^{2}}{\partial t^{2}} \phi=\Delta^{\text {in/out }} \phi
$$

Os modos de freqüência positiva no passado são os projetores espectrais do operador $\Delta^{\text {in }}$, quando realizados em termos das funções $u_{j}$. Já os modos de freqüência positiva no futuro são os projetores espectrais de $\Delta^{\text {out }}$, quando realizados em termos das funções $v_{j^{\prime}}$. É claro que, assintoticamente no passado,

$$
u_{j}(t, \mathbf{x})=\frac{e^{-i \omega_{j} t}}{\sqrt{2 \omega_{j}}} F_{j}(\mathbf{x})
$$

enquanto que, assintoticamente no futuro,

$$
v_{j^{\prime}}(t, \mathbf{x})=\frac{e^{-i \varpi_{j^{\prime}} t}}{\sqrt{2 \varpi_{j^{\prime}}}} G_{j^{\prime}}(\mathbf{x}) .
$$

Entretanto, sem conhecimento da história do espaço-tempo de fundo não é possível saber qual é a forma de $u_{j}$ e $v_{j^{\prime}}$ em outras regiões de $(\mathcal{M}, g)$. Escrevendo, finalmente, os vetores $\varphi_{k}^{\text {in }}$ e $\varphi_{l}^{\text {out }}$, vem

$$
\varphi_{k}^{\text {in }}=\int_{\mathcal{I}} d \mu(j) \tilde{\varphi}_{k}^{\text {in }}(j) u_{j}
$$

$\mathrm{e}$

$$
\varphi_{l}^{\text {out }}=\int_{\mathcal{O}} d \nu\left(j^{\prime}\right) \tilde{\varphi}_{l}^{\text {out }}\left(j^{\prime}\right) v_{j^{\prime}}
$$

Com as expressões Eq. (3.3.9) e Eq. (3.3.10) é possível obter uma relação entre os coeficientes de Bogoliubov, como definidos na Eq. (3.3.2a e na Eq. 3.3.2b), e os modos através da forma do produto interno de KG Eq. (3.1.21). De fato, fazendo a substituição,

$$
A_{k l}=\int_{\mathcal{I}} d \mu(j) \int_{\mathcal{O}} d \nu\left(j^{\prime}\right) \bar{\varphi}_{l}^{\text {out }}\left(j^{\prime}\right) \tilde{\varphi}_{k}^{\text {in }}(j) \alpha_{j j^{\prime}}
$$

$\mathrm{e}$

$$
B_{k l}=\int_{\mathcal{I}} d \mu(j) \int_{\mathcal{O}} d \nu\left(j^{\prime}\right) \tilde{\varphi}_{k}^{\text {in }}(j) \tilde{\tilde{\varphi}}_{l}^{\text {out }}\left(j^{\prime}\right) \beta_{j j^{\prime}}
$$

onde os coeficientes $\alpha_{j j^{\prime}}$ e $\beta_{j j^{\prime}}$ são definidos por

$$
\alpha_{j j^{\prime}}:=-i \Omega\left(\bar{v}_{j^{\prime}}, u_{j}\right)
$$


e

$$
\beta_{j j^{\prime}}:=i \Omega\left(\bar{v}_{j^{\prime}}, \bar{u}_{j}\right)
$$

Na literatura é mais comum ver a denominação coeficiente de Bogoliubov aplicada aos coeficientes $\alpha_{j j^{\prime}}$ e $\beta_{j j^{\prime}}$; ao longo do texto usarei essa denominação tanto para os coeficientes $A_{k l}$ e $B_{k l}$ quanto para os coeficientes $\alpha_{j j^{\prime}}$ e $\beta_{j j^{\prime}}$. Por fim, utilizando a Eq. (3.3.1) com as expressões dadas na Eq. (3.3.9) e na Eq. (3.3.10) e as definições Eq. (3.3.12), também é possível obter a seguinte relação entre os conjuntos de modos $\left\{u_{j}\right\}_{j \in \mathcal{I}}$ e $\left\{v_{j^{\prime}}\right\}_{j^{\prime} \in \mathcal{O}}$ :

$$
u_{j}=\int_{\mathcal{O}} d \mu^{\prime}\left(j^{\prime}\right)\left(\alpha_{j j^{\prime}} v_{j^{\prime}}-\bar{\beta}_{j j^{\prime}} \bar{v}_{j^{\prime}}\right)
$$

A Eq. 3.3.13 é bastante útil para se inferir o comportamento de um conjunto de modos na região futura ou passada se se conhece o comportamento do outro conjunto de modos naquela região.

\subsection{O tensor energia-momentum para o campo quântico}

Uma vez quantizado o campo e definido o espaço de estados do sistema, devemos passar aos observáveis da teoria. Como aqui estou cuidando de um campo livre e estou preocupado com seu papel num sistema gravitacional, é necessário voltar a atenção para o operador tensor energia-momentum, $\hat{T}_{a b}$. Em TQCEC, ao se considerar campos livres, a interação gravitacional passa a ser a única janela de que se dispõe para observar a evolução do sistema quântico. Na literatura é aceito que, em nível semiclássico, o sistema quântico retroage sobre o espaço-tempo segundo a equação de Einstein semiclássica,

$$
R_{a b}-\frac{1}{2} R g_{a b}=8 \pi\left\langle\hat{T}_{a b}\right\rangle
$$

onde $\left\langle\hat{T}_{a b}\right\rangle$ é o valor esperado de $\hat{T}_{a b}$ em algum estado do campo (5-8). Essa equação é usualmente motivada por um paralelo com a abordagem semiclássica para a Eletrodinâmica, mas pode também sê-lo através da dedução formal da correção de ordem mais 
baixa para o campo gravitacional clássico quando a matéria e perturbações sobre $g_{a b}$ são quantizadas. De qualquer forma, não parece haver na literatura uma justificativa última para a equação de Einstein semiclássica a partir da qual fique claro qual deva ser seu regime de validade.

O procedimento de quantização canônica nos diz que para obtermos o operador $\hat{T}_{a b}$ correspondente ao observável clássico $T_{a b}$ devemos substituir em sua expressão o campo $\phi$ pelo correspondente operador de Heisenberg - aqui denotado por $\hat{\phi}$ - e simetrizar o resultado de modo a obtermos um operador auto-adjunto. Entretanto, a Eq. 3.2.2 nos mostra que o tensor energia-momentum é um observável que depende de produtos de campo e suas derivadas no mesmo ponto do espaço-tempo. O fato de $\hat{\phi}$ somente estar definido no sentido de distribuição leva à conclusão de que no contexto do formalismo canônico não é possível definir $\hat{T}_{a b}$ como um operador atuando sobre o espaço de estados do campo ou mesmo dar sentido a ele como distribuição. Esse tipo de problema já aparece em espaço plano e está na raiz da necessidade, tanto no espaço-tempo de Minkowski quanto em espaço-tempos mais gerais, de algum procedimento de renormalização para que se possa dar sentido a quantidades como o tensor energia-momentum.

Para efeitos práticos podemos nos contentar em somente definir o valor esperado de quantidades quadráticas no operador campo, como $\hat{T}_{a b}$ ou as flutuações do campo, dadas pelo valor esperado de $\hat{\phi}^{2}$. Para o tensor energia-momentum, em particular, seu valor esperado em algum estado do campo pode ser definido através de quatro condições, os chamados axiomas de Wald (7). Esses axiomas fixam a forma de $\left\langle\hat{T}_{a b}\right\rangle$ a menos de termos que dependam localmente da geometria de fundo e por isso podem ser absorvidos em constantes, como a de Newton e a cosmológica 14 Os axiomas de Wald, contudo, não fornecem uma prescrição para a construção de $\left\langle\hat{T}_{a b}\right\rangle$. Para tanto, podemos lançar mão do próprio formalismo apresentado. Já foi dito acima que podemos construir $\hat{T}_{a b}$ a partir da expressão para o tensor energia-momentum clássico. Para um estado do campo $\Psi \in \mathscr{F}_{\mathrm{s}}(\mathscr{H})$ qualquer, o valor esperado $\left\langle\Psi\left|\hat{T}_{a b}\right| \Psi\right\rangle:=\left\langle\Psi, \hat{T}_{a b} \Psi\right\rangle_{\mathscr{F}_{\mathrm{s}}(\mathscr{H})}$ será divergente; divergência esta

\footnotetext{
${ }^{14}$ Essa necessidade de contra-termos na ação gravitacional aparece também no formalismo de ação efetiva, onde o comportamento ultravioleta do campo quântico implica em termos que dependem de derivadas da métrica de fundo 43 .
} 
que decorre do fato de que $\hat{T}_{a b}$ está mal definido. Para se obter uma expressão para o valor esperado do tensor energia-momentum com significado físico, $\left\langle\hat{T}_{a b}\right\rangle$, é, então, necessário algum procedimento de regularização e renormalização para "remover os infinitos" de $\left\langle\Psi\left|\hat{T}_{a b}\right| \Psi\right\rangle$. Em linhas gerais, para que os axiomas de Wald sejam satisfeitos, o procedimento de renormalização adotado deve depender somente de propriedades locais do espaço-tempo, ser tal que o resultado final seja covariantemente conservado e que, no caso do espaço-tempo de Minkowski, seja equivalente ao ordenamento normal dos operadores de criação e destruição. Para uma classe especial de estados, conhecidos como estados Hadamard, existe um algoritmo que permite construir $\left\langle\hat{T}_{a b}\right\rangle$ satisfazendo os axiomas de Wald. (Para a construção desse algoritmo veja, e.g., a referência (7).)

Na discussão que se seguirá sobre o Efeito de Despertar do Vácuo, irei tacitamente assumir que o estado inicial do campo é Hadamard. Para o caso particular da formação de um corpo compacto que será discutido no Capítulo 6, por exemplo, o estado inicial do campo é Hadamard, o que está implícito na escolha dos modos in lá feita. 


\section{Capítulo 4}

\section{Um estudo da equação de}

\section{Klein-Gordon}

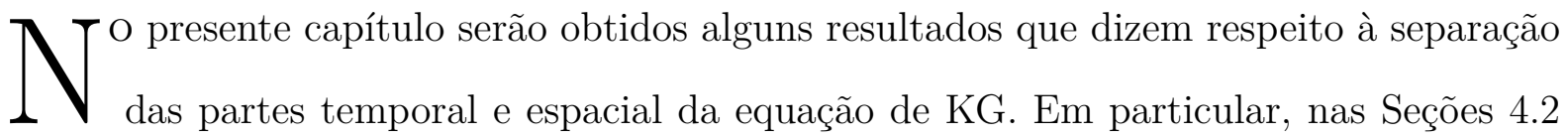

e 4.3 paulatinamente explorarei possíveis simetrias do espaço-tempo de fundo, as quais serão usadas mais adiante para construir a linha principal de argumentação. Os resultados obtidos e as análises feitas aqui serão utilizadas no Capítulo 5 para derivar o crescimento sem-limites da densidade de energia de vácuo de um campo quântico escalar em espaçostempos bastante gerais e analisar tal fenômeno.

\subsection{Decomposição $3+1$ da equação de Klein-Gordon}

Como já foi argumentado no Capítulo 3 o procedimento de quantização passa pela escolha do espaço de Hilbert $\mathscr{H}$. Em particular, em espaços-tempos estacionários ou estáticos há uma escolha natural para $\mathscr{H}$ baseada na isometria temporal do espaço-tempo de fundo. No caso estático foi mostrado que as autofunções e o espectro do operador $\Delta$ são peças importantes para o entendimento do operador campo e seus observáveis. Portanto, é interessante ao menos obtermos algumas propriedades qualitativas das soluções complexas 
da Eq. (3.1.2) em alguns espaços-tempos.

Vou começar por um espaço-tempo $(\mathcal{M}, g)$ globalmente hiperbólico geral. Tendo em vista as definições feitas na seção 2.1, é possível escrever a derivada de uma função escalar qualquer $\phi$ da seguinte forma:

$$
\nabla_{a} \phi=-n_{a}\left(n^{b} \nabla_{b} \phi\right)+D_{a} \phi
$$

Tomando o 4-divergente da Eq. 4.1.1 e escrevendo a métrica $g_{a b}$ em termos de $h_{a b}$ e $n^{a}$, vem a forma do operador de Laplace-Beltrami em termos de objetos definidos na seção espacial de $(\mathcal{M}, g)$ :

$$
\nabla_{a} \nabla^{a} \phi=-n^{a} \nabla_{a}\left(n^{b} \nabla_{b} \phi\right)-K n^{a} \nabla_{a} \phi+\mathrm{a}^{a} D_{a} \phi+D_{a} D^{a} \phi
$$

Então, usando a Eq. 4.1.2), vem a forma decomposta da equação de KG,

$$
-n^{a} \nabla_{a}\left(n^{b} \nabla_{b} \phi\right)-K n^{a} \nabla_{a} \phi+\mathrm{a}^{a} D_{a} \phi+D_{a} D^{a} \phi-\left(m^{2}+\xi R\right) \phi=0 .
$$

Uma vez que desejo estudar as soluções complexas para a equação de campo, é necessário definir a função escalar $\phi$ como

$$
\phi: \mathcal{M} \rightarrow \mathbb{C}
$$

Para soluções complexas a corrente

$$
J_{a}:=-i\left(\phi \nabla_{a} \bar{\phi}-\bar{\phi} \nabla_{a} \phi\right)
$$

é conservada pela dinâmica do campo $\bigsqcup^{1}$ Sendo $\phi$ uma função complexa, posso decompô-la como

$$
\phi=\rho e^{-i \varphi},
$$

onde as funções $\rho$ e $\varphi$ são definidas como

$$
\rho: \mathcal{M} \rightarrow \mathbb{R}_{+},
$$

\footnotetext{
${ }^{1}$ Para o campo de KG carregado, a corrente $J_{a}$ descreve a corrente de cargas. Nesta tese, entretanto, não estou interessado em campo carregados.
} 


$$
\varphi: \mathcal{M} \rightarrow \mathbb{R}
$$

Substituindo a Eq. (4.1.6) na Eq. (3.1.2) vem que a Eq. (3.1.2) é satisfeita se, e somente se,

$$
\left\{\begin{array}{l}
-\nabla_{a} \nabla^{a} \rho+\nabla_{a} \varphi \nabla^{a} \varphi+\left(m^{2}+\xi R\right) \rho=0 \\
-\rho \nabla_{a} \nabla^{a} \varphi-2 \nabla_{a} \rho \nabla^{a} \varphi=0
\end{array} .\right.
$$

A segunda equação na Eq. 4.1.8 pode ser integrada um vez. Vejamos. Multiplicando por $\rho$, vem que

$$
\nabla_{a}\left(\rho^{2} \nabla^{a} \varphi\right)=0
$$

Então, sendo $\mathcal{N}$ a porção de $\mathcal{M}$ entre $\Sigma_{t_{1}}$ e $\Sigma_{t_{2}}$,

$$
0=\int_{\mathcal{N} \subset \mathcal{M}} \nabla_{a}\left(\rho^{2} \nabla^{a} \varphi\right) \sqrt{-g} d^{4} x=\int_{\Sigma_{t_{2}}} \rho^{2} \nabla_{a} \varphi n^{a} d \Sigma-\int_{\Sigma_{t_{1}}} \rho^{2} \nabla_{a} \varphi n^{a} d \Sigma
$$

onde assumi que o campo anula-se no infinito espacial e foi empregado o Teorema de Stokes. Esse resultado nada mais é do que a conservação da corrente $J_{a}$ definida na Eq. 4.1.5. Substituindo a Eq. 4.1.1) e a Eq. 4.1.2 na Eq. 4.1.8 vem o seguinte par de equações:

$$
\begin{aligned}
&-n^{a} \nabla_{a}(\left.n^{b} \nabla_{b} \rho\right)-K n^{a} \nabla_{a} \rho+\mathrm{a}^{a} D_{a} \rho+D_{a} D^{a} \rho \\
&-\left[m^{2}+\xi R+D_{a} \varphi D^{a} \varphi-\left(n^{a} \nabla_{a} \varphi\right)^{2}\right] \rho=0,
\end{aligned}
$$

e

$$
\begin{aligned}
&-n^{a} \nabla_{a}\left(n^{b} \nabla_{b} \varphi\right)-K n^{a} \nabla_{a} \varphi-n^{a} \nabla_{a} \ln \rho^{2} n^{a} \nabla_{a} \varphi \\
&+\mathrm{a}^{a} D_{a} \varphi+D_{a} \ln \rho^{2} D^{a} \varphi+D_{a} D^{a} \varphi=0 .
\end{aligned}
$$

Considere também o tensor energia-momentum, definido na Eq. 3.2.2, associado a um perfil de campo complexo $\phi$. O escalar $n^{a} n^{b} T_{a b}$ pode ser manipulado de modo a escrevê-lo em termos de objetos definidos na Seção 2.2, de sorte que é possível obter a 
seguinte igualdade:

$$
\begin{aligned}
n^{a} n^{b} T_{a b}= & \frac{1}{4} n^{a} \nabla_{a} \phi n^{b} \nabla_{b} \bar{\phi}+\frac{1-4 \xi}{4}\left[D_{a} \phi D^{a} \bar{\phi}+\left(m^{2}+\xi R\right) \phi \bar{\phi}\right] \\
& +\xi\left[\phi K n^{a} \nabla_{a} \bar{\phi}-\phi D_{a} D^{a} \bar{\phi}+\left(m^{2}+\xi R\right) \phi \bar{\phi}\right] \\
& +\frac{\xi}{2} n^{a} n^{b} R_{a b} \phi \bar{\phi}+\text { c.c. },
\end{aligned}
$$

onde foi empregada a equação de campo na forma da Eq. 4.1.3). O mesmo tipo de procedimento pode ser aplicado ao campo vetorial $h^{a b} n^{c} T_{b c}$, para o qual é possível mostrar que

$$
\begin{aligned}
h^{a b} n^{c} T_{b c} & =\frac{1-2 \xi}{2} D^{a} \phi n^{c} \nabla_{c} \bar{\phi}+\frac{\xi}{2} h^{a b} n^{c} R_{b c} \phi \bar{\phi} \\
& -\xi\left[D^{a}\left(n^{c} \nabla_{c} \phi\right) \bar{\phi}-K^{a c} D_{c} \phi \bar{\phi}\right]+\text { c.c. }
\end{aligned}
$$

Essas duas quantidades são interessantes pois estão associadas com a densidade de energia e de corrente de energia do campo e que, como veremos adiante, satisfazem uma equação de conservação quando o espaço-tempo de fundo possui uma isometria temporal.

\subsection{Decomposição $3+1$ da equação de Klein-Gordon em um espaço-tempo estático}

Como já mencionado na Seção 2.1, quando o espaço-tempo é estático podemos escolher o campo de Killing tipo tempo como vetor coordenado. Essa escolha da coordenada temporal implica que os coeficientes que comparecem na Eq. 3.1.2 independem de $t$. Assim, a equação de KG admite soluções na forma da Eq. 4.1.6 com

$$
\begin{gathered}
\rho=T X \\
\varphi=\varphi_{t}+\varphi_{\Sigma},
\end{gathered}
$$

onde as funções acima são definidas como segue:

$$
T: \mathbb{R} \rightarrow \mathbb{R}_{+}
$$




$$
\begin{gathered}
X: \Sigma \rightarrow \mathbb{R}_{+}, \\
\varphi_{t}: \mathbb{R} \rightarrow \mathbb{R}, \\
\varphi_{\Sigma}: \Sigma \rightarrow \mathbb{R} .
\end{gathered}
$$

Então, substituindo a Eq. 4.2.1 e a Eq. 4.2.2 na Eq. 4.1.11a e na Eq. 4.1.11b e usando a Eq. 2.2.12 e que

$$
\varkappa^{a} \nabla_{a}\left(\varkappa^{b} \varkappa_{b}\right)=0
$$

vem o seguinte par de equações:

$$
\begin{aligned}
-\frac{1}{T} \frac{1}{\|\varkappa\|^{2}} \varkappa^{a} \nabla_{a}\left(\varkappa^{b} \nabla_{b} T\right)+\frac{\left(\varkappa^{a} \nabla_{a} \varphi_{t}\right)^{2}}{\|\varkappa\|^{2}}+\frac{1}{X} D_{a} D^{a} X & \\
+\frac{1}{X} \mathrm{a}^{a} D_{a} X-\left(m^{2}+\xi R+D_{a} \varphi_{\Sigma} D^{a} \varphi_{\Sigma}\right) & =0 \\
-\frac{1}{\|\varkappa\|^{2}} \varkappa^{a} \nabla_{a}\left(\varkappa^{b} \nabla_{b} \varphi_{t}\right)-\frac{2}{T} \frac{1}{\|\varkappa\|^{2}}\left(\varkappa^{a} \nabla_{a} T\right)\left(\varkappa^{b} \nabla_{b} \varphi_{t}\right) & \\
+\mathrm{a}^{a} D_{a} \varphi_{\Sigma}+\frac{2}{X} D_{a} X D^{a} \varphi_{\Sigma}+D_{a} D^{a} \varphi_{\Sigma} & =0 .
\end{aligned}
$$

Neste ponto, aplicando o método de separação de variáveis ao par de equações acima, obtém-se que

$$
\begin{gathered}
\varkappa^{a} \nabla_{a}\left(\varkappa^{b} \nabla_{b} T\right)-\left(\varkappa^{a} \nabla_{a} \varphi_{t}\right)^{2} T+\sigma T=0, \\
D_{a} D^{a} X+\mathrm{a}^{a} D_{a} X-\left(m^{2}+\xi R+D_{a} \varphi_{\Sigma} D^{a} \varphi_{\Sigma}\right) X+\frac{\sigma}{\|\varkappa\|^{2}} X=0, \\
T^{2} \varkappa^{a} \nabla_{a}\left(\varkappa^{b} \nabla_{b} \varphi_{t}\right)+\left(\varkappa^{a} \nabla_{a} T^{2}\right)\left(\varkappa^{b} \nabla_{b} \varphi_{t}\right)+\lambda T^{2}=0, \\
X^{2} D_{a} D^{a} \varphi_{\Sigma}+\mathrm{a}^{a} D_{a} \varphi_{\Sigma} X^{2}+D_{a} X^{2} D^{a} \varphi_{\Sigma}+\frac{\lambda}{\|\varkappa\|^{2}} X^{2}=0 .
\end{gathered}
$$

Por $\sigma$ e $\lambda$ denoto, respectivamente, as constantes de separação entre a variável temporal e as variáveis espaciais da Eq. 4.1.11a e da Eq. 4.1.11b.

Vou agora manipular a Eq. 4.2.7c e a Eq. 4.2.7d para mostrar que é possível fixar o valor de $\lambda$ usando a condição de contorno no infinito espacial. Primeiro, note que é 
possível, usando a Eq. (2.2.13), colocar a Eq. (4.2.7d) na seguinte forma:

$$
D^{a}\left[\|\varkappa\| X^{2} D_{a} \varphi_{\Sigma}\right]+\lambda \frac{X^{2}}{\|\varkappa\|^{2}}=0 .
$$

Integrando a expressão acima dos dois lados em alguma hiperfície $\Sigma_{t}$, segue que

$$
\int_{\Sigma_{t}} D^{a}\left[\|\varkappa\| X^{2} D_{a} \varphi_{\Sigma}\right] d \Sigma+\lambda \int_{\Sigma_{t}} \frac{X^{2}}{\|\varkappa\|^{2}} d \Sigma=0
$$

A primeira integral é nula pelo Teorema de Stokes e pelo fato de que estou assumindo que $X$ anula-se no infinito espacial. A segunda parcela tem como integrando uma quantidade estritamente positiva. Logo, a expressão acima é verdade, para $X$ não identicamente nulo em $\Sigma$, se, e somente se, $\lambda=0$. Levando esse fato para a Eq. $4.2 .7 \mathrm{c}$, , segue que

$$
T^{2} \varkappa^{a} \nabla_{a} \varphi_{t} \equiv \text { cte. }
$$

Usando a carga conservada pela corrente $J_{a}$, tem-se, para uma solução $\phi$ escrita na forma da Eq. 4.1.6), que

$$
\int_{\Sigma_{t}} n^{a} J_{a} d \Sigma=\int_{\Sigma_{t}} \rho^{2} n^{a} \nabla_{a} \varphi d \Sigma=2 T^{2} \varkappa^{a} \nabla_{a} \varphi_{t} \int_{\Sigma_{t}} \frac{X^{2}}{\|\varkappa\|} d \Sigma \equiv \text { cte }
$$

o que é consistente com o fato de que $J_{a}$ é conservado.

Através da forma da Eq. 4.2.10 e da Eq. 4.2.7a vou analisar a relação entre a amplitude de uma solução complexa da equação de KG no caso estático e os sinais da constante de separação $\sigma$. Para tanto, escreva

$$
\varkappa^{a} \nabla_{a} \varphi_{t}=\frac{C}{T^{2}}
$$

onde $C$ é uma constante real. Substituindo essa relação na Eq. 4.2.7a em favor de $T$ e multiplicando o resultado por $\varkappa^{c} \nabla_{c} T$, vem

$$
\varkappa^{a} \nabla_{a}\left[\frac{1}{2}\left(\varkappa^{b} \nabla_{b} T\right)^{2}+\frac{1}{2} \frac{C^{2}}{T^{2}}+\frac{\sigma}{2} T^{2}\right]=0
$$

e o argumento da derivada independe de $t$ :

$$
\left(\varkappa^{b} \nabla_{b} T\right)^{2}+\frac{C^{2}}{T^{2}}+\sigma T^{2}=\mathcal{E}
$$


onde $\mathcal{E}$ é uma constante real. Pode-se, então, definir o seguinte "potencial":

$$
\vartheta(T)=\frac{C^{2}}{T^{2}}+\sigma T^{2}
$$

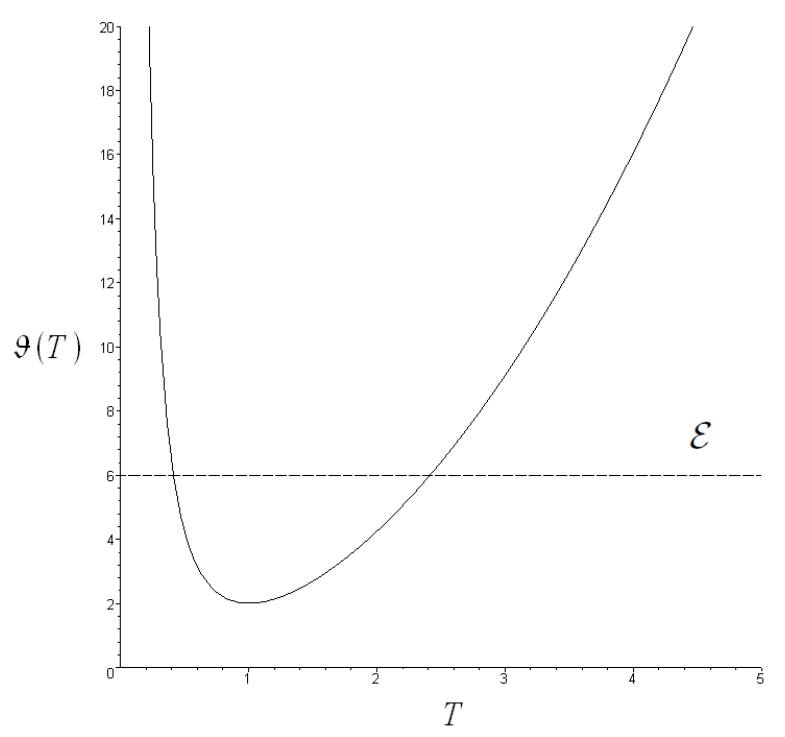

(a)

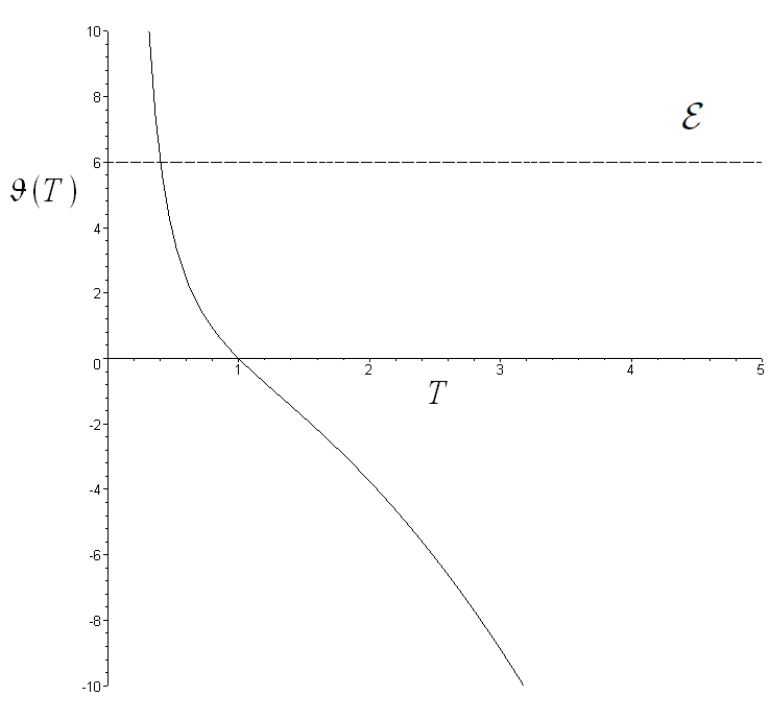

(b)

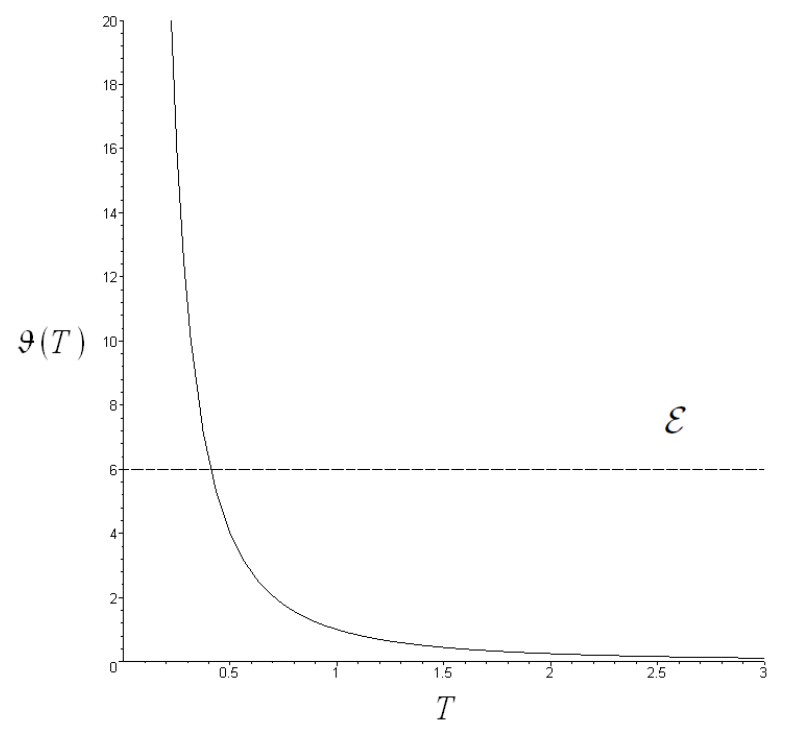

(c)

Figura 4.1 - Gráficos da função potencial Eq. 4.2.15 para $C \neq 0$. No gráfico (a) fixei $\sigma>0$, em (b) $\sigma<0$ e em (c) $\sigma=0$. A linha pontilhada denota, ao cruzar com a linha cheia, os "pontos de retorno" do potencial.

Da Fig. 4.1 (a) vê-se que todas as soluções possíveis para a Eq. 4.2.7a quando $\sigma>0$ são funções limitadas na coordenada t. Por outro lado, se $\sigma \leq 0-$ Fig. 4.1 (b) e (c) a Eq. 4.2.7a admite soluções que crescem arbitrariamente. Note que quando a norma da solução da equação de campo é nula, a função da Eq. 4.2.15 tem os comportamentos representados na Fig. 4.2, dependendo do sinal de $\sigma$. Assim, se $\sigma>0$, a função $T$ é 


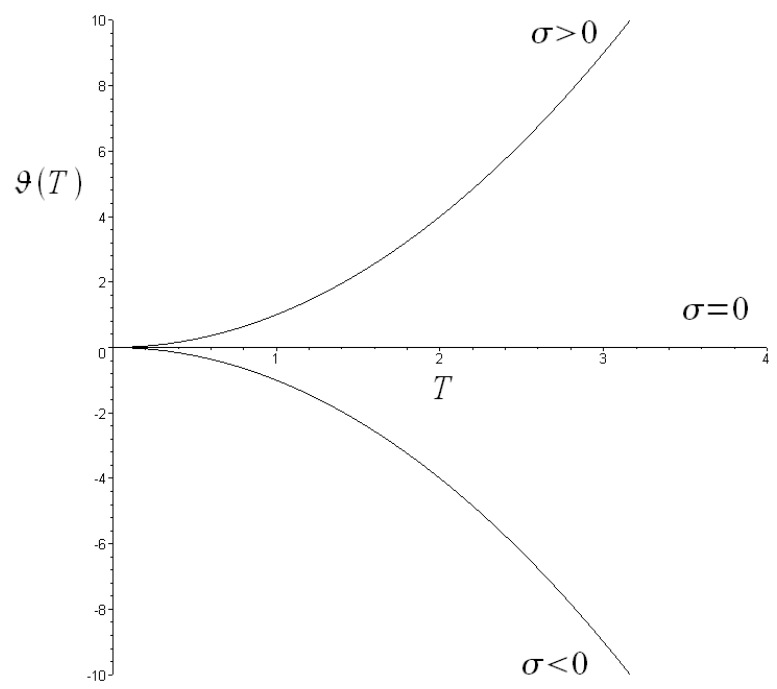

Figura 4.2 - Gráfico da função potencial Eq. 4.2.15 fixado $C=0$ para $\sigma>0, \sigma<0$ e $\sigma=0$.

limitada enquanto que, se $\sigma \leq 0, T$ cresce ou vai a zero. Em particular, se $\sigma<0$ e $\mathcal{E}=0$ há soluções da Eq. 4.2.7a que tendem a zero num tempo infinito.

Por fim, voltemos às Eqs. 4.2.7a-4.2.7d). Já mostrei que a condição de contorno que diz que os perfis de campo devem anular-se no infinito implica que a constante de separação $\lambda$ deve ser zero. Esse fato permite que a escolha $\varphi_{\Sigma}=0$ seja consistente com a Eq. $4.2 .7 \mathrm{~d}$ em todo aberto de $\Sigma_{t}$ no qual $\rho \neq 0.2^{2}$ Com essa escolha e a expressão da Eq. 2.2.13 para o campo a ${ }^{a}$, é possível colocar a Eq. 4.2.7b na seguinte forma:

$$
-\|\varkappa\| D^{a}\left(\|\varkappa\| D_{a} X\right)+\|\varkappa\|^{2}\left(m^{2}+\xi R\right) X=\sigma X
$$

Essa forma para a equação satisfeita por $X$ será útil mais adiante. O operador diferencial que comparece na Eq. 4.2.16 é a forma prometida para o operador $\Delta$ na Seção 3.2 É importante destacar que o comportamento temporal de uma solução complexa arbitrária da equação de campo num espaço-tempo estático foi relacionada no parágrafo anterior com os possíveis valores da constante de separação $\sigma$. Quais são esses valores, entretanto, é uma pergunta cuja resposta depende exclusivamente da Eq. 4.2.16), isto é, depende de qual será o conjunto de $\sigma$ para o qual a Eq. 4.2.16) apresentará soluções condizentes com as condições de contorno e constituem o espectro de $\Delta$.

\footnotetext{
${ }^{2}$ Por razões práticas, então, é mais simples relaxar a definição de $X$, usando $\mathbb{R}$ como seu contra-domínio. Isso será feito mais adiante.
} 
Para propósitos futuros, também é interessante explorar a forma das quantidades da Eq. 4.1.12 e da Eq. 4.1.13 quando o perfil de campo pode ser colocado na forma $T e^{-i \varphi_{t}} X$. Utilizando os resultados da Eq. (2.2.12) e Eq. (2.2.13) e a Eq. (4.2.16), além da relação

$$
\varkappa^{a} \varkappa^{b} R_{a b} \phi \bar{\phi}=\|\varkappa\| D_{a}\left(D^{a}\|\varkappa\| \phi \bar{\phi}\right)-\|\varkappa\| D_{a}\|\varkappa\| D^{a}(\phi \bar{\phi})
$$

que é obtida a partir da definição do tensor de Riemann Eq. (2.1.2), é possível colocar a Eq. 4.1.12 na seguinte forma:

$$
\begin{aligned}
n^{a} n^{b} T_{a b}= & \frac{1}{2} \frac{\left|\varkappa^{a} \nabla_{a}\left(T e^{-i \varphi t}\right)\right|^{2}}{\|\varkappa\|^{2}} X^{2}+\frac{T^{2}}{\|\varkappa\|^{2}}\left[\| \varkappa \| D _ { a } \left(\xi D^{a}\|\varkappa\| X^{2}\right.\right. \\
& \left.\left.+\frac{1-4 \xi}{4}\|\varkappa\| D^{a} X^{2}\right)+\frac{1}{2} \sigma X^{2}\right]
\end{aligned}
$$

Já para o campo vetorial $h^{a b} n^{c} T_{b c}$ é possível mostrar, usando Eq. 2.2.12) e que

$$
h^{a b} \varkappa^{c} R_{b c} \phi \bar{\phi}=0
$$

que

$$
h^{a b} n^{c} T_{b c}=\frac{\varkappa^{c} \nabla_{c} T^{2}}{\|\varkappa\|^{2}}\left(\frac{1-4 \xi}{4}\|\varkappa\| D^{a} X^{2}+\xi D^{a}\|\varkappa\| X^{2}\right)
$$

Ademais, a 4-divergência nula de $T_{a b}$ dada na Eq. (3.2.3) contraída com o campo de Killing tipo tempo, implica na seguinte equação de conservação:

$$
\varkappa^{a} \nabla_{a}\left(\|\varkappa\| n^{b} n^{c} T_{b c}\right)+D_{a}\left(-\|\varkappa\|^{2} h^{a b} n^{c} T_{b c}\right)=0
$$

Logo, em um espaço-tempo estático, é razoável definir a densidade de energia associada ao perfil $\phi$ e sua corrente, respectivamente, por

$$
\rho_{\phi}:=\|\varkappa\| n^{a} n^{b} T_{a b}
$$

e

$$
j_{\phi}^{a}:=-\|\varkappa\|^{2} h^{a b} n^{c} T_{b c} .
$$




\subsection{Decomposição $3+1$ da equação de Klein-Gordon em um espaço-tempo estático e esfericamente simétrico}

Vejamos, por fim, como podemos prosseguir com a decomposição da equação de KG quando dispomos de um espaço-tempo estático e esfericamente simétrico. Para tanto, posso usar o campo vetorial $\left(e_{r}\right)^{a}$ para decompor as seções espaciais de $(\mathcal{M}, g)$, como já mencionado na Seção 2.1. A decomposição do operador diferencial $D_{a}$ na Eq. 4.2.16) e o fato de que, como o espaço-tempo é esfericamente simétrico, o campo a ${ }^{a}$ tem componente somente na direção de $\left(e_{r}\right)^{a}$, levam a $\mathrm{I}^{3}$

$$
\begin{aligned}
-\|\varkappa\|\left(e_{r}\right)^{a} D_{a}\left[\|\varkappa\|\left(e_{r}\right)^{b} D_{b} X\right]-\|\varkappa\|^{2} \Theta_{a} \Theta^{a} X & \\
-\|\varkappa\|^{2} S\left(e_{r}\right)^{a} D_{a} X+\|\varkappa\|^{2}\left(m^{2}+\xi R\right) X & =\sigma X .
\end{aligned}
$$

Para simplificar a equação acima, vou escrever $X$ como

$$
X=\frac{E}{U}
$$

e vou escolher $U$ de modo que $\Theta_{a} U=0$, ou seja, constante sobre cada esfera gerada pelas isometrias, e

$$
\left(e_{r}\right)^{a} D_{a} U=\frac{1}{2} S U
$$

onde $S$, o traço da curvatura extrínseca dessas esferas, foi definido pela Eq. (2.2.18). Para encontrar a equação para $E$ que decorre da Eq. (4.3.1) basta substituir a Eq. (4.3.2) na Eq. (4.3.1) e no resultado usar a Eq. 4.3.3. Assim, segue para E que

$$
\begin{gathered}
-\|\varkappa\|\left(e_{r}\right)^{a} D_{a}\left[\|\varkappa\|\left(e_{r}\right)^{b} D_{b} E\right]-\|\varkappa\|^{2} \Theta_{a} \Theta^{a} E \\
+\left\{\|\varkappa\|^{2}\left(m^{2}+\xi R\right)+\frac{1}{U}\|\varkappa\|\left(e_{r}\right)^{a} D_{a}\left[\|\varkappa\|\left(e_{r}\right)^{b} D_{b} U\right]\right\} E=\sigma E .
\end{gathered}
$$

\footnotetext{
${ }^{3} \mathrm{~A}$ afirmação de que $\mathrm{a}^{a}$ só tem componente na direção de $\left(e_{r}\right)^{a}$ pode ser provada contraindo o campo $\mathrm{a}^{a}$ com os campos que geram as isometrias sobre as superfícies esféricas e utilizando-se a equação de Killing. Essa é a expressão rigorosa do fato geometricamente intuitivo de que um campo vetorial esfericamente simétrico tem que ser puramente radial.
} 
Por último, induzido pela forma da derivada na direção de $\left(e_{r}\right)^{a}$ na equação acima, definirei o seguinte campo vetorial:

$$
\left(\partial_{\chi}\right)^{a}:=\|\varkappa\|\left(e_{r}\right)^{a} .
$$

Pela forma com que foi definido acima, $\left(\partial_{\chi}\right)^{a}$ é um vetor coordenado. De fato, uma vez que $\left(\partial_{\chi}\right)^{a}$ comuta com os campos de Killing que geram a isometria temporal e as isometrias que definem as superfícies esféricas. Isso pode ser mostrado recorrendo-se a definição dada na Eq. (2.2.14) e usando a equação de Killing. A coordenada radial $\chi$ será útil mais adiante

\footnotetext{
${ }^{4}$ No espaço-tempo de um buraco negro $\chi$ é conhecida na literatura como coordenada de tartaruga de Wheeler (1), usualmente denotada por $r^{*}$.
} 


\section{Capítulo 5}

\section{Do mecanismo de despertar do vácuo}

$\mathrm{N}^{\prime \prime}$

ESTE capítulo discorrerei sobre o Efeito de Despertar do Vácuo em espaços-tempos

conformalmente estáticos. O primeiro passo será escrever o operador $\hat{\Phi}$ em termos dos modos da equação de KG. Isso será feito ao longo da Seção 5.1 . Na Seção 5.2 argumento do porquê a existência de modos exponencialmente crescentes no futuro implicam num crescimento sem-limites das flutuações e da densidade de energia do campo no estado de vácuo. Com a finalidade de obter uma condição necessária para o desencadeamento do efeito, exploro na Seção 5.3 um guia para o aparecimento dos modos com comportamento exponencial. Por fim, na Seção 5.4 calculo a contribuição dos graus de liberdade do campo descritos por esses modos ao valor esperado da densidade e corrente de energia do campo e sua energia total.

\subsection{Quantização do campo}

Para argumentar em favor da afirmação de que existem espaços-tempos bem-comportados capazes, por conta da evolução, de forçar a densidade de energia de vácuo de um campo quântico a se tornar protagonista na evolução do sistema vou utilizar um espaço-tempo conformalmente estático. Seja $(\mathcal{M}, g)$ um espaço-tempo gerado por alguma distribuição 
de matéria clássica tal que sua métrica possa ser escrita como

$$
g_{a b}=\Psi^{2} \tilde{g}_{a b},
$$

onde $\tilde{g}_{a b}$ admite um campo de Killing $\tilde{\varkappa}^{a}$ tipo tempo e existe uma foliação $\left\{\Sigma_{t}\right\}_{t \in \mathbb{R}}$ tal que $\tilde{n}^{a}:=\frac{\tilde{\mathcal{\varkappa}}^{a}}{\|\tilde{\mathcal{\imath}}\|}$ é normal às hiperfícies $\Sigma_{t}$. Aqui $\Psi: \mathcal{M} \rightarrow \mathbb{R}_{+}^{*}$ é uma função suave chamada de fator conforme. Substituindo essa forma para $g_{a b}$ na equação de KG Eq. (3.1.2 e a reescrevendo em termos de objetos definidos no espaço-tempo $(\mathcal{M}, \tilde{g})$ e o fator conforme, tem-se que

$$
-\tilde{g}^{a b} \tilde{\nabla}_{a} \tilde{\nabla}_{b} \tilde{\phi}+\left(\Psi^{2} m^{2}+\xi \tilde{R}+\frac{1-6 \xi}{\Psi} \tilde{g}^{a b} \tilde{\nabla}_{a} \tilde{\nabla}_{b} \Psi\right) \tilde{\phi}=0
$$

onde

$$
\phi=\frac{\tilde{\phi}}{\Psi}
$$

O operador derivada $\tilde{\nabla}_{a}$ é aquele compatível com $\tilde{g}_{a b}$ e $\tilde{R}$ é o escalar de curvatura relacionado ao espaço-tempo $(\mathcal{M}, \tilde{g})$. Vou agora aplicar a decomposição $3+1$ para o espaçotempo $\left(\mathcal{M}, \tilde{g}_{a b}\right)$. Usando o resultado da Eq. 4.1.2 na primeira parcela do lado esquerdo da Eq. (5.1.2), vem que

$$
-\tilde{\varkappa}^{a} \tilde{\nabla}_{a}\left(\tilde{\varkappa}^{b} \tilde{\nabla}_{b} \tilde{\phi}\right)=-\|\tilde{\varkappa}\| \tilde{D}_{a}\left(\|\tilde{\varkappa}\| \tilde{D}^{a} \tilde{\phi}\right)+V_{\text {ef }} \tilde{\phi}
$$

onde

$$
V_{\mathrm{ef}}:=\|\tilde{\mathcal{H}}\|^{2}\left(\Psi^{2} m^{2}+\xi \tilde{R}+\frac{1-6 \xi}{\Psi} \tilde{g}^{a b} \tilde{\nabla}_{a} \tilde{\nabla}_{b} \Psi\right)
$$

O operador derivada $\tilde{D}_{a}$ é aquele compatível com o tensor métrico $\tilde{h}_{a b}$, induzido nas seções espaciais pela foliação escolhida.

Uma situação relativamente simples na qual o efeito já pode ser desencadeado é a seguinte. Suponha que o espaço-tempo $(\mathcal{M}, g)$ é tal que tanto assintoticamente no passado quanto assintoticamente no futuro $V_{\text {ef }}$ não depende da variável temporal definida pelo campo de Killing $\tilde{\varkappa}^{a}$. Essa simplificação abrange campos sem massa em espaços-tempos que são assintoticamente estáticos tanto no passado como no futuro, caso da formação de uma estrela estática a partir de matéria espalhada por todo o espaço, e campos sem massa e com acoplamento conforme. Desse modo é possível proceder com o esquema de 
quantização do campo escalar $\phi$ como apresentado nas Seções 3.1 e 3.2 . Outra conseqüência dessa simplificação é fazer com que, tanto no passado quanto no futuro, seja possível implementar uma decomposição dos modos da equação de campo aos moldes do que foi feito para uma solução complexa arbitrária, via o método de separação de variáveis, na Seção 4.2. Aqui, entretanto, a equação satisfeita por $X$ é

$$
-\|\tilde{\varkappa}\| \tilde{D}_{a}\left(\|\tilde{\varkappa}\| \tilde{D}^{a} X\right)+V_{\mathrm{ef}} X=\sigma X
$$

com $V_{\text {ef }}$ dado pela Eq. 5.1.5.

Vou começar pelo estudo dos modos que são de freqüência positiva assintoticamente no passado. Suponha que nessa região a Eq. (5.1.6) admita soluções que não divergem no infinito espacial somente para $\sigma$ positivo. Logo, como argumentado na Seção 3.2 , na região passada devemos escolher a parte temporal dos modos de sorte que

$$
i £_{\tilde{\varkappa}} u=\omega u,
$$

com $\omega \in \mathbb{R}_{+}$. Implementando para os modos $u$ a decomposição Eq. 4.1.6 com $\rho$ e $\varphi$, respectivamente, com as formas da Eq. (4.2.1) e da Eq. (4.2.2), vem que

$$
-i T \tilde{\varkappa}^{a} \tilde{\nabla}_{a} \varphi_{t}+\tilde{\varkappa}^{a} \tilde{\nabla}_{a} T=-i \omega T .
$$

Essa relação é satisfeita escolhendo $T=\frac{1}{\sqrt{2 \omega}}$ e $\varphi_{t}=\omega t$. Substituindo essas escolhas na Eq. 4.2.7a segue que $\omega=\sigma^{1 / 2}$. Por fim, o conjunto de modos de freqüência positiva satisfazendo as condições acima no passado, $\left\{u_{j}\right\}_{j \in \mathcal{I}}$ tem como elementos

$$
u_{j}=\frac{e^{-i \omega_{j} t}}{\sqrt{2 \omega_{j}}} \frac{F_{j}}{\Psi}
$$

por $F_{j}: \Sigma_{t \rightarrow-\infty} \rightarrow \mathbb{C}$ denoto as soluções da Eq. (5.1.6) no passado. Note que a escolhas feitas para $T$ e $\varphi_{t}$ têm o propósito de fazer com que

$$
-i \Omega\left(\bar{u}_{j}, u_{j^{\prime}}\right)=\delta_{\mu}\left(j, j^{\prime}\right)
$$

onde $\Omega$ denota a forma simplética definida na Eq. (3.1.5) e $\delta_{\mu}$, assim como antes, é tal que $\int_{\mathcal{I}} d \mu(j) \delta\left(j, j^{\prime}\right)=1$, com $\mu(j)$ uma medida definida sobre $\mathcal{I}$. Assim, $\hat{\phi}$ pode ser escrito 
como

$$
\hat{\phi}=\sum_{k \in I}\left(\varphi_{k}^{\text {in }} \hat{a}_{k}+\bar{\varphi}_{k}^{\text {in }} \hat{a}_{k}^{\dagger}\right)
$$

onde

$$
\varphi_{k}^{\text {in }}=\int_{\mathcal{I}} d \mu(j) \tilde{\varphi}_{k}^{\text {in }}(j) u_{j}
$$

que denota os elementos da base de $\mathscr{H}_{\text {in }}$. Vou escolher como condição inicial para o campo quântico $\hat{\Phi}$ o estado de vácuo in, isto é, o vetor $\Psi_{0}^{\text {in }} \in \mathscr{F}_{\mathrm{s}}\left(\mathscr{H}_{\text {in }}\right)$ que, assim como antes, satisfaz

$$
\hat{a}_{k} \Psi_{0}^{\text {in }}=0
$$

para todo $k \in I$.

Para conhecermos o comportamento do sistema quântico no futuro temos de, ao menos a princípio, ser capazes de evoluir os modos $u_{j}$ através da equação de campo até aquela região do espaço-tempo. Obviamente, devido à evolução do sistema gravitacional, no futuro a base de modos $\left\{u_{j}\right\}_{j \in \mathcal{I}}$ não será mais de freqüência positiva. Por conta disso, faz-se conveniente escolher um outro conjunto de modos. Diferentemente do que supus no passado, vou considerar agora que no futuro o espaço-tempo é tal que existem soluções da Eq. (5.1.6) para a qual $\sigma$ é negativo e que não diverge no infinito espacial. O fato de que assintoticamente no futuro o espaço-tempo ser tal que $V_{\text {ef }}$ não depende da coordenada temporal permite, para as autofunções da Eq. (5.1.6) com $\sigma>0$, uma decomposição em termos de modos da equação de campo naquela região escolhidos segundo a mesma receita seguida para o passado. Esses modos têm a seguinte forma:

$$
v_{l}=\frac{e^{-i \varpi_{l} t}}{\sqrt{2 \varpi_{l}}} \frac{G_{l}}{\Psi}
$$

$\operatorname{com} l \in \mathcal{O}$, um outro conjunto de índices. Aqui as funções $G_{l}: \Sigma_{t \rightarrow+\infty} \rightarrow \mathbb{C}$ são soluções da Eq. (5.1.6) no futuro para $\sigma>0$. Para os modos cuja parte espacial satisfazem a Eq. 5.1.6 com $\sigma<0$ não é possível, contudo, escolher sua dependência temporal de maneira que satisfaçam a Eq. (5.1.7) assintoticamente no futuro. Dito de outra forma, as escolhas para $T$ e $\varphi_{t}$ feitas para os modos com $\sigma>0$ não são consistentes com o fato de que sua parte espacial satisfaz a Eq. (5.1.6) com $\sigma<0$. Uma maneira de encontrar a forma 
da parte temporal desses modos é resolver a Eq. 4.2.14 para $\sigma<0$. Para tanto, vou escrevê-la da seguinte forma:

$$
\frac{d T}{d t}= \pm \frac{\sqrt{|\sigma| T^{4}+\mathcal{E} T^{2}-C}}{T}
$$

o que leva a

$$
t= \pm \frac{1}{2} \frac{1}{|\sigma|^{1 / 2}} \int_{\operatorname{arccosh} y_{0}}^{\operatorname{arccosh} y} d u
$$

onde $y=\frac{2|\sigma| T^{2}+\mathcal{E}}{\sqrt{4|\sigma| C^{2}+\mathcal{E}^{2}}}$ e $y_{0}=\frac{2|\sigma| T_{0}^{2}+\mathcal{E}}{\sqrt{4|\sigma| C^{2}+\mathcal{E}^{2}}}$. A expressão acima implica que, como função de $t, T^{2}$ se escreve como

$$
T^{2}=\frac{\sqrt{4|\sigma| C^{2}+\mathcal{E}^{2}}}{2|\sigma|} \cosh \left[2|\sigma|^{1 / 2}(t-\theta)\right]-\frac{\mathcal{E}}{2|\sigma|},
$$

onde $\theta$ e $\mathcal{E}$ são constantes que devem ser escolhidas nas condições iniciais. Note que escolhi o sinal + na expressão para $T^{2}$; uma vez que cosh é uma função par, o último resultado é independente dessa escolha. A propósito de simplificar a forma da expressão final, vou tomar

$$
\mathcal{E}=-|\sigma|^{1 / 2} \cot (2 \alpha), \theta=0 \text { e } C=\frac{1}{2}
$$

e assim vem que

$$
T^{2}=\frac{\cosh \left(2|\sigma|^{1 / 2} t\right)+\cos (2 \alpha)}{2|\sigma|^{1 / 2} \operatorname{sen}(2 \alpha)} .
$$

Ainda é necessário determinar a fase da parte temporal do modo. Sua expressão pode ser obtida através da equação diferencial para a fase, Eq. (4.2.12), cujo resultado é

$$
\varphi_{t}=\arctan \left[\tan \alpha \tanh \left(|\sigma|^{1 / 2} t\right)\right]
$$

Por fim, tem-se que a forma da parte temporal dos modos cuja parte espacial é solução da Eq. (5.1.6) para $\sigma<0$ é

$$
T e^{-i \varphi_{t}}=\frac{e^{\Omega t-i \alpha}+e^{-\Omega t+i \alpha}}{\sqrt{4 \Omega \operatorname{sen}(2 \alpha)}},
$$

onde $\Omega=|\sigma|^{1 / 2}$ e $\left.\left.\alpha \in\right] 0, \frac{\pi}{4}\right]$. Logo, assintoticamente no futuro, pelo fato de que estou supondo que existem soluções da Eq. (5.1.6) para $\sigma<0$, é necessário, além do conjunto 
de modos $\left\{v_{l}\right\}_{l \in \mathcal{O}}$, o conjunto de modos $\left\{w_{n}^{\left(\alpha_{n}\right)}\right\}_{n \in \mathcal{O}^{\prime}}$, cujos elementos têm a forma

$$
w_{n}^{\left(\alpha_{n}\right)}=\frac{e^{\Omega_{n} t-i \alpha_{n}}+e^{-\Omega_{n} t+i \alpha_{n}}}{\sqrt{4 \Omega_{n} \operatorname{sen}\left(2 \alpha_{n}\right)}} \frac{H_{n}}{\Psi} .
$$

Denoto por $H_{n}: \Sigma_{t \rightarrow+\infty} \rightarrow \mathbb{C}$ as soluções da Eq. (5.1.6) no futuro para $\sigma<0$. Para ser coerente com as referências (24,25), ao longo do texto assumirei $\alpha_{n}=\pi / 12$, para todo $n \in \mathcal{O}^{\prime}$, exceto se apontado diferente. Sendo assim, a fim de simplificar a notação, suprimirei a menção ao parâmetro $\alpha_{n}$ - a não ser que isso possa levar a alguma confusão. Portanto, em termos desses outros conjuntos, temos que

$$
\hat{\phi}=\sum_{k^{\prime} \in O}\left(\varphi_{k^{\prime}}^{\text {out }} \hat{b}_{k^{\prime}}+\bar{\varphi}_{k^{\prime}}^{\text {out }} \hat{b}_{k^{\prime}}^{\dagger}\right)
$$

onde

$$
\varphi_{k^{\prime}}^{\text {out }}=\int_{\mathcal{O}} d \nu(l) \tilde{\varphi}_{k^{\prime}}^{\text {out }}(l) v_{l}+\int_{\mathcal{O}^{\prime}} d \nu^{\prime}(n) \tilde{\varphi}_{k^{\prime}}^{\text {out }}(n) w_{n}
$$

que denota os elementos da base de $\mathscr{H}_{\text {out }}$ É possível verificar que, assim como os operadores $\hat{a}$ e $\hat{a}^{\dagger}$ definidos acima, os operadores $\hat{b}$ e $\hat{b}^{\dagger}$ satisfazem as relações de comutação dadas na Eq. 3.1.40.

\subsection{Do crescimento ilimitado das flutuações e suas implicações}

A questão que se põe neste ponto é a seguinte: de que maneira a evolução do operador campo sobre o espaço-tempo afeta a física do sistema como observada na região assintoticamente no futuro? A maneira de responder a essa indagação é através do cálculo do valor esperado no estado inicial do tensor energia-momentum. Foi mencionado na Seção 3.4 que esse observável é um operador quadrático no operador campo, de modo que faz-se mister algum processo de regularização e renormalização para que o resultado obtido tenha significado físico. À parte o problema de renormalização, o cálculo desse valor esperado passa por saber como os operadores de criação e destruição construídos a partir da base out se escrevem em termos daqueles definidos a partir da base in. Tal rela- 
ção depende exclusivamente da evolução dos modos $u_{j}$ pela equação de campo. No caso geral não é de se esperar que modos de freqüência positiva no futuro possam ser escritos somente em termos de modos de freqüência positiva no passado. Esse fato implica nos já conhecidos efeitos de criação de partículas por campos gravitacionais como em casos cosmológicos ou no Efeito Hawking. Desejo, contudo, chamar atenção às conseqüências da existência do conjunto de modos $\left\{w_{n}\right\}_{n \in \mathcal{O}^{\prime}}$. Uma vez que tais modos também são imprescindíveis para se escrever perfis arbitrários do campo no futuro, há elementos do conjunto $\left\{u_{j}\right\}_{j \in \mathcal{I}}$ que têm projeção não-nula nos modos que no futuro crescem exponencialmente. Inevitavelmente, então, modos com a forma da Eq. 5.1.22 contribuirão para o valor esperado das flutuações do campo e do tensor energia-momentum naquela região do espaço-tempo. Em outras palavras, a divergência assintótica desses modos leva ao crescimento sem-limites das flutuações do campo no estado inicial — mesmo sendo este bem-comportado no passado - de maneira que em termos da parcela dominante

$$
\left\langle\hat{\phi}^{2}\right\rangle \stackrel{\text { futuro }}{\sim} \frac{\kappa e^{2 \bar{\Omega} t}}{2 \bar{\Omega}}\left(\frac{|\bar{H}|}{\Psi}\right)^{2}\left[1+\mathcal{O}\left(e^{-\epsilon t}\right)\right],
$$

onde as quantidades com barra sobre referem-se ao menor valor possível de $\sigma$ para o qual a Eq. (5.1.6) tem solução não-divergente no infinito espacial. $\epsilon$ é uma constante positiva e $\kappa$ é uma constante sem dimensão, da ordem da unidade e que depende globalmente do espaço-tempo, uma vez que está relacionada com a projeção da base in na base out. Essa explosão nas flutuações do campo também reflete-se no comportamento do valor esperado do tensor energia-momentum sobre o espaço-tempo $(\mathcal{M}, g)$ :

$$
\begin{aligned}
&\left\langle\hat{T}_{00}\right\rangle \stackrel{\text { futuro }}{\sim}\left\langle\hat{\phi}^{2}\right\rangle\left\{\frac{(1-4 \xi)}{2}\left(\bar{\Omega}^{2}+\frac{|\tilde{D} \bar{H}|^{2}}{|\bar{H}|^{2}}+m^{2} \Psi^{2}\right)\right. \\
&+(1-6 \xi)\left(\frac{2 \xi \ddot{\Psi}}{\Psi}-\frac{2 \xi \tilde{D}^{2} \Psi}{\Psi}+\frac{\dot{\Psi}^{2}}{2 \Psi^{2}}-\frac{\bar{\Omega} \dot{\Psi}}{\Psi}\right. \\
&\left.\left.+\frac{(\tilde{D} \Psi)^{2}}{2 \Psi^{2}}-\operatorname{Re} \frac{\tilde{D}_{i} \Psi \tilde{D}^{i} \bar{H}}{\Psi \bar{H}}\right)+\mathcal{O}\left(e^{-\epsilon t}\right)\right\}, \\
&\left\langle\hat{T}_{0 i}\right\rangle \stackrel{\text { futuro }}{\sim}\left\langle\hat{\phi}^{2}\right\rangle\left\{(1-4 \xi) \operatorname{Re} \frac{\bar{\Omega} \tilde{D}_{i} \bar{H}}{\bar{H}}+(1-6 \xi)\left(\frac{\dot{\Psi} \tilde{D}_{i} \Psi}{\Psi^{2}}\right.\right. \\
&\left.\left.-\operatorname{Re} \frac{\dot{\Psi} \tilde{D}_{i} \bar{H}}{\Psi \bar{H}}-\frac{\bar{\Omega} \tilde{D}_{i} \Psi}{\Psi}\right)+\mathcal{O}\left(e^{-\epsilon t}\right)\right\},
\end{aligned}
$$




$$
\begin{aligned}
\left\langle\hat{T}_{i j}\right\rangle \stackrel{\text { futuro }}{\sim} & \left\langle\hat{\phi}^{2}\right\rangle\left\{(1-2 \xi) \operatorname{Re} \frac{\tilde{D}_{i} \bar{H} \tilde{D}_{j} \bar{H}}{\bar{H}^{2}}-2 \xi \operatorname{Re} \frac{\tilde{D}_{i} \tilde{D}_{j} \bar{H}}{\bar{H}}+\xi \tilde{R}_{i j}\right. \\
& +\frac{(1-4 \xi) \tilde{h}_{i j}}{2}\left(\bar{\Omega}^{2}-\frac{|\tilde{D} \bar{H}|^{2}}{|\bar{H}|^{2}}-m^{2} \Psi^{2}\right) \\
& +(1-6 \xi)\left[\frac{\tilde{D}_{i} \Psi \tilde{D}_{j} \Psi}{\Psi^{2}}-\operatorname{Re} \frac{\tilde{D}_{i} \Psi \tilde{D}_{j} \bar{H}}{\Psi \bar{H}}-\operatorname{Re} \frac{\tilde{D}_{j} \Psi \tilde{D}_{i} \bar{H}}{\Psi \bar{H}}\right. \\
& +\tilde{h}_{i j}\left(\frac{2 \xi \tilde{D}^{2} \Psi}{\Psi}-\frac{2 \xi \ddot{\Psi}}{\Psi}+\frac{\dot{\Psi}^{2}}{2 \Psi^{2}}-\frac{\bar{\Omega} \dot{\Psi}}{\Psi}-\frac{(\tilde{D} \Psi)^{2}}{2 \Psi^{2}}\right. \\
& \left.\left.\left.+\operatorname{Re} \frac{\tilde{D}_{k} \Psi \tilde{D}^{k} \bar{H}}{\Psi \bar{H}}\right)\right]+\mathcal{O}\left(e^{-\epsilon t}\right)\right\} .
\end{aligned}
$$

Aqui $\tilde{h}_{i j}$ são as componentes do tensor $\tilde{h}_{a b}$. $\tilde{R}_{i j}$ denota as componentes espaciais do tensor de Ricci calculado a partir da métrica $\tilde{g}_{a b}$ e $\dot{\Psi}$ corresponde à derivada do fator conforme em relação à coordenada temporal. As Eqs. 5.2.2a - 5.2.2c), juntamente com a Eq. (5.2.1), revelam que uma vez disparada, a explosão da densidade de energia do vácuo passa a rivalizar com a densidade de energia clássica numa escala de tempo relacionada a $\bar{\Omega}^{-1}$. A partir daí, o destino do sistema físico composto pelo sistema gravitacional mais campo quântico somente pode ser inferido através da equação de Einstein semiclássica.

\subsection{Uma condição necessária para o Efeito de Despertar do Vácuo}

Ainda resta, todavia, argumentar o porquê é razoável considerar situações nas quais a Eq. 5.1.6 admite soluções para $\sigma<0$. Vejamos. Observando a Eq. 5.1.4 vemos que, nas regiões assintóticas do espaço-tempo físico, a questão dos possíveis sinais de $\sigma$ é justamente a questão de encontrar autofunções do operador

$$
-\|\tilde{\varkappa}\| \tilde{D}_{a}\left(\|\tilde{\varkappa}\| \tilde{D}^{a} \cdot\right)+V_{\mathrm{ef}}
$$

e seu espectro. Em particular, a tarefa de encontrar modos com $\sigma<0$ pode ser fraseada nos seguintes termos: sob quais condições um poço de potencial permite estados com autovalor negativo. Da experiência com Mecânica Quântica sabemos que para a equação 
de Schrödinger independente do tempo há um bom guia para antecipar a existência desses estados. Se o poço é profundo o suficiente em uma certa região de sorte que o produto entre o tamanho típico da região e a profundidade típica do potencial seja da ordem de um, então esse é um sistema promissor para se encontrar estados ligados $1^{1}$ A grosso modo, isso é verdade pois a região do poço deve ser grande o suficiente para que ali pelo menos caiba um comprimento de onda e pelo fato de que, se existirem, a energia típica dos estados ligados é da mesma ordem da profundidade do poço. Para o caso da Eq. (5.1.4, o potencial efetivo $V_{\text {ef }}$ tem dimensão de energia ao quadrado e portanto para ela o bom guia é $\left|V_{\text {ef }}\right| L^{2} \sim 1$, onde $L$ é o tamanho típico da região espacial na qual o potencial efetivo é negativo. Pela forma do potencial efetivo dada na Eq. (5.1.5), nota-se que é perfeitamente possível, manipulando o parâmetro $\xi$ e tomando a massa do campo igual a zero, que este se torne negativo por uma região suficientemente grande para que permita modos $\operatorname{com} \sigma<0$.

Mas o quão natural são os cenários que permitem que $V_{\text {ef }}$ fique suficientemente negativo? Para responder a esse questionamento vou assumir que $\xi \sim 1, m=0$ e a equação de Einstein para a métrica de fundo. Nesse caso, o potencial efetivo é da ordem do escalar de curvatura $R$. Se $\rho_{\text {class }}$ é a densidade de energia clássica, fonte para o campo gravitacional, então, pela equação de Einstein, vem que $R \sim G \rho_{\text {class }} / c^{2}$, ao se recuperar a constante de Newton e a velocidade da luz. O guia que evoquei acima diz que em cenários nos quais a densidade $\rho_{\text {class }}$ numa região de tamanho típico $L$ satisfaça

$$
\begin{aligned}
\frac{G \rho_{\text {class }} L^{2}}{c^{2}} & \approx \frac{\rho_{\text {class }}}{10^{15} \mathrm{~g} / \mathrm{cm}^{3}}\left(\frac{L}{7 \mathrm{~km}}\right)^{2} \\
& \approx \frac{\rho_{\text {class }}}{2.5 \times 10^{-30} \mathrm{~g} / \mathrm{cm}^{3}}\left(\frac{L}{4.7 \times 10^{3} \mathrm{Mpc}}\right)^{2} \sim 1
\end{aligned}
$$

há chances de o efeito ser desencadeado. Na condição acima já explicitei dois cenários nos quais é promissora a possibilidade de o efeito desempenhar algum papel: astrofísicos e cosmológicos, respectivamente. No primeiro cenário comparece a densidade típica de estrelas de nêutrons $\left(10^{15}-10^{17} \mathrm{~g} / \mathrm{cm}^{3}\right)$, sistemas em cujo interior o potencial efetivo

\footnotetext{
${ }^{1}$ Em uma ou duas dimensões sempre existe estado ligado para a equação de Schrödinger, independentemente da profundidade do poço de potencial, se a integral do potencial é negativa. Veja a referência (44).
} 
pode se tornar negativo. No segundo cenário comparece a densidade média de matéria bariônica mais matéria escura numa região com tamanho típico da ordem do comprimento de Hubble, $4.1 \times 10^{3} \mathrm{Mpc}$.

Como já mencionado, o efeito fornece uma escala de tempo típica para que a densidade de energia do vácuo passe a rivalizar com a densidade de energia do sistema clássico que gera o espaço-tempo de fundo. Fraseando o efeito em termos de um potencial efetivo, é possível estimar a ordem de grandeza dessa escala. Tipicamente, $\bar{\Omega}$ é da ordem da raiz quadrada da profundidade do poço, isto é,

$$
\bar{\Omega} \sim \sqrt{\frac{G \rho_{\text {class }}}{c^{2}}} .
$$

Então, a escala de tempo para que o efeito se torne macroscopicamente relevante é

$$
\tau \sim \sqrt{\frac{1}{G \rho_{\text {class }}}} \approx \sqrt{\frac{10^{15} \mathrm{~g} / \mathrm{cm}^{3}}{\rho_{\text {class }}}} \times 10^{-4} \mathrm{~s} \approx \sqrt{\frac{2.5 \times 10^{-30} \mathrm{~g} / \mathrm{cm}^{3}}{\rho_{\text {class }}}} \times 10^{10} \text { anos. }
$$

Na expressão acima escrevo a escala de tempo em termos da densidade típica, respectivamente, de cenários astrofísicos (estrelas de nêutrons) e cosmológicos. Enquanto que em cenários cosmológicos o desencadeamento do efeito levaria da ordem de bilhões de anos para desempenhar papel relevante, em estrelas de nêutrons essa escala é da ordem de frações de milissegundos.

Tendo em vista a discussão desta seção e os cenários concretos que serão expostos no Capítulo 6, é razoável supor que os valores de $\sigma$ negativos compõem o espectro pontual do operador Eq. 5.3.1) ou, em outras palavras, que as autofunções $H_{n}$ são autovetores daquele operador. Isso permite escolher a base out de maneira que alguns de seus elementos sejam os próprios modos $w_{n}$. Ademais, uma vez que são funções localizadas, é possível escolher $H_{n}$ de forma que este seja uma função real. A fim de guiar os olhos do leitor, denotarei a partir de agora por $\hat{c}_{n}$ e $\hat{c}_{n}^{\dagger}$, respectivamente, os operadores de destruição e criação associados aos modos $w_{n}$. 


\subsection{Da energia associada ao mecanismo}

Até este ponto argumentei em favor da afirmação de que existem espaços-tempos bemcomportados nos quais, assintoticamente no futuro, graus de liberdade de um campo quântico são descritos por modos que crescem exponencialmente. Vejamos agora como se comporta a energia associada a esses modos nessa região do espaço-tempo.

Com os resultados da Seção 4.2 para o campo escalar $n^{a} n^{b} T_{a b}$ e o campo vetorial $h^{a b} n^{c} T_{b c}$, obtidos a partir do tensor energia-momentum associado a um perfil de campo complexo, podemos nos perguntar qual a forma da densidade de energia e corrente quando o perfil $\phi$ é um modo exponencialmente crescente. Nesse caso, $\sigma=-\Omega^{2}$ e $T e^{-i \varphi_{t}}$ é dado na Eq. (5.1.21). Fazendo, então, as devidas substituições na Eq. 4.2.18), vem

$$
\begin{aligned}
n^{a} n^{b} T_{a b}= & \frac{1}{\|\varkappa\|}\left(\frac{e^{2 \Omega t}+e^{-2 \Omega t}}{2 \Omega}+\frac{\cos \frac{\pi}{6}}{\Omega}\right) D_{a}\left(\xi D^{a}\|\varkappa\| X^{2}+\frac{1-4 \xi}{4}\|\varkappa\| D^{a} X^{2}\right) \\
& -\Omega \cos \frac{\pi}{6} \frac{X^{2}}{\|\varkappa\|^{2}} .
\end{aligned}
$$

As mesmas substituições na Eq. 4.2.20 levam a

$$
h^{a b} n^{c} T_{b c}=\frac{e^{2 \Omega t}-e^{-2 \Omega t}}{\|\varkappa\|^{2}}\left(\frac{1-4 \xi}{4}\|\varkappa\| D^{a} X^{2}+\xi D^{a}\|\varkappa\| X^{2}\right) .
$$

As expressões Eq. (5.4.1) e Eq. (5.4.2), mais a equação de conservação da Eq. 4.2.21, sugerem a seguinte imagem mental do mecanismo. As flutuações de vácuo do campo são perturbadas pelo campo gravitacional do sistema de matéria clássica de modo que este força o aparecimento de uma corrente de energia do vácuo por todo o espaço. Isso faz com que a densidade de energia torne-se cada vez mais positiva em algumas regiões da seção espacial enquanto que em outras essa densidade torna-se cada vez mais negativa. A localização dessas regiões depende do espaço-tempo e do acoplamento $\xi$ do campo com o escalar de curvatura. Esse processo vai até o ponto em que a densidade de energia do vácuo acumulada nessas regiões fica comparável à densidade de energia clássica que gerou o espaço-tempo em primeiro lugar. Como já foi mencionado, a partir desse ponto é necessário levar em conta a retroação do campo quântico sobre o espaço-tempo para se 
decidir o destino do sistema.

A despeito desse selvagem rearranjo da distribuição da energia do vácuo no futuro, o valor esperado da energia total do campo quântico é conservado. De fato, é possível mostrar que formalmente o operador hamiltoniano, quando escrito em termos dos operadores de criação e destruição da base out, tem a forma

$$
\begin{aligned}
\hat{H}:= & \int_{\Sigma} d \Sigma\|\varkappa\| n^{a} n^{b} \hat{T}_{a b} \\
= & \frac{1}{2} \sum_{k, k^{\prime} \in O}\left(\hat{b}_{k}^{\dagger} \hat{b}_{k^{\prime}}+\hat{b}_{k} \hat{b}_{k^{\prime}}^{\dagger}\right) \int_{\mathcal{O}} d \nu(j) \bar{\varphi}_{k}^{\text {out }}(j) \tilde{\varphi}_{k^{\prime}}^{\text {out }}(j) \varpi_{j} \\
& -\frac{\sqrt{3}}{2} \sum_{n \in \mathcal{O}^{\prime}}\left(\hat{c}_{n}^{\dagger} \hat{c}_{n}+\hat{c}_{n} \hat{c}_{n}^{\dagger}\right) \Omega_{n}-\sum_{n \in \mathcal{O}^{\prime}}\left(\hat{c}_{n} \hat{c}_{n}+\hat{c}_{n}^{\dagger} \hat{c}_{n}^{\dagger}\right) \Omega_{n},
\end{aligned}
$$

para a qual é necessário expressar os elementos da base out em termos dos modos $v_{l}$ e $w_{n}$. O valor esperado de $\hat{H}$ no vácuo in fica

$$
\begin{aligned}
\left\langle 0_{\text {in }}|\hat{H}| 0_{\text {in }}\right\rangle:= & \left\langle\Psi_{0}^{\text {in }}, \hat{H} \Psi_{0}^{\text {in }}\right\rangle_{\mathscr{F}_{\mathrm{s}}\left(\mathscr{G}_{\text {in }}\right)} \\
= & \frac{1}{2} \sum_{\substack{k, k^{\prime} \in O \\
l \in I}}\left(A_{l k} \bar{A}_{l k^{\prime}}+B_{l k} \bar{B}_{l k^{\prime}}\right) \int_{\mathcal{O}} d \nu(j) \tilde{\varphi}_{k}^{\text {out }}(j) \tilde{\varphi}_{k^{\prime}}^{\text {out }}(j) \varpi_{j} \\
& -\frac{\sqrt{3}}{2} \sum_{\substack{n \in \mathcal{O}^{\prime} \\
l \in I}}\left(\left|A_{l n}\right|^{2}+\left|B_{l n}\right|^{2}\right) \Omega_{n} \\
& +\sum_{\substack{n \in \mathcal{O}^{\prime} \\
l \in I}}\left(A_{l n} B_{l n}+\bar{A}_{l n} \bar{B}_{l n}\right) \Omega_{n}
\end{aligned}
$$

onde fiz uso das transformações de Bogoliubov, como definida na Seção 3.3. A primeira parcela da Eq. (5.4.4 refere-se à contribuição da energia de vácuo dos modos $v_{k}$ mais a eventual produção de quanta desses modos por conta da evolução do sistema gravitacional. Naturalmente, essas contribuições são positivas. Já as segunda e terceira parcelas dão conta da contribuição dos modos exponenciais ao valor esperado da energia. Note que diferentemente da primeira contribuição, essa pode ser negativa. 


\section{Capítulo 6}

\section{O mecanismo em espaços-tempos particulares}

To que segue discorrerei sobre o aparecimento do efeito exposto no capítulo anterior

1 em dois espaços-tempos particulares. Aqui em momento algum a retroação do campo quântico sobre a densidade de matéria clássica que gera o espaço-tempo em questão será levada em conta.

Na Seção 6.1 mostro que estrelas de nêutrons, os objetos mais densos de que temos conhecimento, são capazes de despertar o vácuo. Já na Seção 6.2, o efeito será derivado no espaço-tempo gerado por uma casca esféria estática. Apesar de ser um espaço-tempo idealizado, este último tem a vantagem de permitir algumas análises interessantes. Isso, pois, nesse caso é possível conhecer parte do comportamento da contribuição dominante do efeito ao valor esperado do tensor energia-momentum do campo.

\subsection{Despertando o vácuo no interior de estrelas}

O cenário ao qual me aterei aqui é o da formação de um objeto astrofísico que assintoticamente no futuro é estático e esfericamente simétrico. Por simplicidade, suponha que no 
passado a matéria que comporá o objeto está toda espalhada pelo espaço. Assim, no passado o espaço-tempo é muito bem aproximado pelo de Minkowski. Com essas hipóteses, podemos escrever a forma do tensor métrico:

$$
g_{a b} \sim \begin{cases}-(d t)_{a}(d t)_{b}+(d x)_{a}(d x)_{b}+(d y)_{a}(d y)_{b}+(d z)_{a}(d z)_{b}, & \text { passado } \\ -f\left[(d t)_{a}(d t)_{b}-(d \chi)_{a}(d \chi)_{b}\right]+s_{a b}, & \text { futuro }\end{cases}
$$

com

$$
s_{a b}:=r^{2}\left[(d \theta)_{a}(d \theta)_{b}+\operatorname{sen}^{2} \theta(d \varphi)_{a}(d \varphi)_{b}\right]
$$

Os sistemas de coordenadas utilizados são definidos como segue. No passado usei a construção padrão em Minkowski: um sistema de coordenadas cartesiano baseado nas interpretações de tempo e distância de uma família de observadores inerciais. No futuro utilizei o campo de Killing tipo tempo $\varkappa^{a}$ daquela região para definir o vetor coordenado temporal. As coordenadas $\theta$ e $\varphi$ são aquelas que cobrem as superfícies esféricas, a menos dos dois pólos de cada uma delas. A coordenada radial $\chi$ é definida pelo vetor coordenado $\left(\partial_{\chi}\right)^{a}$ dado na Eq. 4.3.5. As funções $f=f(\chi):=-\varkappa^{c} \varkappa_{c}>0$ e $r=r(\chi) \geq 0$ são funções somente de $\chi$ e são tais que $f(\chi) \rightarrow 1$ e $r(\chi) / \chi \rightarrow 1$ quando $\chi \rightarrow \infty$. Além disso, $d r / d \chi>0$. Essas condições são impostas para que o espaço-tempo no futuro não possua horizonte de eventos ou superfícies de aprisionamento de cones de luz e seja plano no infinito espacial. É importante notar que, a despeito do fato de eu estar usando a mesma letra para denotar a coordenada temporal no passado e no futuro, os t's em cada uma das regiões têm interpretações diferentes.

Considere agora um campo quântico escalar $\hat{\Phi}$ que obedece à Eq. 3.1.2. Uma vez que o espaço-tempo é estático no passado e torna-se estático novamente no futuro, podemos nos valer dos argumentos dados nos capítulos anteriores para construir a quantização do campo de KG. No passado, o conjunto de modos, $\left\{u_{\mathbf{k}}\right\}_{\mathbf{k} \in \mathbb{R}^{3}}$, tem elementos com a forma

$$
u_{\mathbf{k}} \stackrel{\text { passado }}{\sim} \frac{1}{\sqrt{16 \pi^{3} \omega_{\mathbf{k}}}} e^{i\left(\mathbf{k} \cdot \mathbf{x}-\omega_{\mathbf{k}} t\right)}
$$

sendo $\omega_{\mathbf{k}}:=\sqrt{\mathbf{k}^{2}+m^{2}}$. Já no futuro, a parte espacial dos modos obedece à Eq. 4.3.4. Vejamos, então, a forma de $U$ em termos das coordenadas usadas nessa região. Pela 
definição de $S$ vem que

$$
S=D_{a}\left(e_{r}\right)^{a}=\frac{1}{\sqrt{h}} \partial_{i}\left[\sqrt{h}\left(e_{r}\right)^{i}\right]=\frac{2}{\sqrt{f} r} \frac{d r}{d \chi} .
$$

Substituindo esse resultado na Eq. 4.3 .3 , multiplicada por $\sqrt{f}$, segue

$$
U=r
$$

Assim, em termos das coordenadas no futuro, Eq. 4.3.4 se escreve:

$$
-\frac{\partial^{2}}{\partial \chi^{2}} E-\frac{f}{r^{2}}\left[\frac{1}{\operatorname{sen} \theta} \frac{\partial}{\partial \theta}\left(\operatorname{sen} \theta \frac{\partial}{\partial \theta} E\right)+\frac{1}{\operatorname{sen}^{2} \theta} \frac{\partial^{2}}{\partial \varphi^{2}} E\right]+\left[f\left(m^{2}+\xi R\right)+\frac{1}{r} \frac{d^{2} r}{d \chi^{2}}\right] E=\sigma E .
$$

Pela forma dos operadores diferenciais que comparecem na equação acima, é possível separar as variáveis angulares da variável radial. Impondo que a parte angular dos modos deva ser regular e univocamente definida sobre as superfícies esféricas, $E$ pode ser escrito como

$$
E_{\sigma l \mu}=\psi_{\sigma l} Y_{l \mu}
$$

$Y_{l \mu}$ são os harmônicos esféricos $(l=0,1, \ldots$ e $\mu=-l, \ldots, l)$ e $\psi_{\sigma l}$, função somente de $\chi$, é solução da seguinte equação:

$$
-\frac{d^{2}}{d \chi^{2}} \psi_{\sigma l}+V_{\mathrm{ef}}^{(l)} \psi_{\sigma l}=\sigma \psi_{\sigma l}
$$

a partir de onde estou definindo o seguinte potencial efetivo $V_{\mathrm{ef}}^{(l)}$ :

$$
V_{\mathrm{ef}}^{(l)}:=f\left[m^{2}+\xi R+\frac{l(l+1)}{r^{2}}\right]+\frac{1}{r} \frac{d^{2} r}{d \chi^{2}} .
$$

Assim como antes, coloca-se a questão de descobrir quais são os sinais possíveis para $\sigma$, o que somente pode ser respondido estudando a forma do potencial efetivo.

Vou modelar a matéria que forma a estrela por um fluido ideal. Logo, a matéria estelar no futuro é descrita por duas funções: sua densidade $\rho$ e sua pressão $P$. Por conta das simetrias impostas ao espaço-tempo, estas funções somente dependem da variável radial e $P$ deve ser exatamente a pressão necessária para contrabalancear a força gravitacional de modo a manter o corpo estático. Através da equação de Einstein Eq. 2.1.5), é possível relacionar as componentes da métrica no futuro com o conteúdo de energia do sistema 
clássico. Isso permite escrever o potencial efetivo em termos de $\rho$ e $P$ :

$$
V_{\mathrm{ef}}^{(l)}=f\left[m^{2}+\frac{l(l+1)}{r^{2}}+(\xi-1 / 6) R+\frac{8 \pi G}{3}(\bar{\rho}-\rho)\right],
$$

sendo que $\bar{\rho}$, a densidade média de matéria da estrela até um certo raio, é definido aqui por

$$
\bar{\rho}(\chi):=\frac{3 M(\chi)}{4 \pi r^{3}(\chi)}
$$

com $M(\chi)$ a massa do corpo até $\chi$. Vale lembrar que, segundo a equação de Einstein, o escalar de curvatura satisfaz $R=8 \pi G(\rho-3 P)$. Vejamos a Eq. 6.1.10). Em primeiro lugar, note que as duas primeiras parcelas são positivas. Em particular, é claro que a possibilidade de o efeito ser disparado é potencialmente maior para os modos $\operatorname{com} l=0$. O segundo ponto é o seguinte. De modo geral, para que a Eq. 6.1.8 admita soluções para $\sigma<0$ é necessário que $V_{\text {ef }}^{(l)}$ seja suficientemente negativo, o que passa, para valores de $|\xi| \sim 1$, por $m^{2} \ll R \sim G \rho$. Para densidades usuais de estrelas de nêutrons $\left(10^{15}-10^{17} \mathrm{~g} / \mathrm{cm}^{3}\right)$, ao recuperar as unidades em que massa e densidade são usualmente expressas, vê-se que essa relação entre a massa do campo e a densidade da estrela pode ser posta na forma

$$
\frac{m^{2} /\left(3.5 \times 10^{-11} \mathrm{eV}\right)^{2}}{\rho /\left(10^{15} \mathrm{~g} / \mathrm{cm}^{3}\right)} \ll 1 .
$$

A despeito de sua contribuição positiva, é possível, então, que campos maciços apresentem modos com crescimento sem-limites. Entretanto, a escala de massa para a qual isso é verdade é tão pequena que as únicas partículas que temos conhecimento e cuja massa satisfaz a relação acima são aquelas com $m=0$. Por essa razão, nos resultados que seguirão, assumirei um campo sem massa. Por último, suponha um campo com $\xi=1 / 6$. Logo, a altura do potencial efetivo está diretamente relacionado com as flutuações de densidade do objeto astrofísico. Uma vez que para um objeto desse tipo é de se esperar que $\rho$ decresça conforme se vai de seu centro para sua superfície, segue que $\bar{\rho}-\rho>0$ para todo $\chi$. Logo, nesse espaço-tempo, o efeito nunca é disparado para campos com acoplamento conforme.

Para prosseguir com a tarefa de mostrar que a formação de um objeto astrofísico com- 
pacto é capaz de perturbar o vácuo de forma tão dramática, faz-se mister procurar por soluções da Eq. (6.1.8) com $\sigma<0$ para alguns perfis de densidade para os quais o espaçotempo é estável na ausência de qualquer outro campo. Isso é feito da seguinte forma. Uma vez dado o perfil de densidade, resolve-se a equação de Tolman-Oppenheimer-Volkoff (TOV) para o equilíbrio hidrostático relativístico afim de se obter $P$ e, conseqüentemente, $f$ (veja, e.g., a referência (4)). A partir dessas quantidades, constrói-se o potencial efetivo $V_{\mathrm{ef}}^{(l)}$ e procura-se, numericamente, por "estados ligados" da Eq. 6.1.8 com autovalores negativos. A estratégia para fazer essa procura foi a seguinte. Escolhendo um $\sigma$ suficientemente negativo e fixada a condição de contorno em $\chi=0$, é sempre possível fazer com que a solução da Eq. (6.1.8) divirja para $\chi \rightarrow+\infty$ com o mesmo sinal que tem sobre a superfície da estrela. Suponha que para $\sigma=0$ a solução diverge, quando $\chi \rightarrow+\infty$, com sinal trocado em relação àquele sobre a superfície da estrela. Isso significa que em algum momento, para algum $\sigma<0$ intermediário a solução "troca de sinal" no infinito, isto é, vai a zero quando $\chi \rightarrow+\infty$.

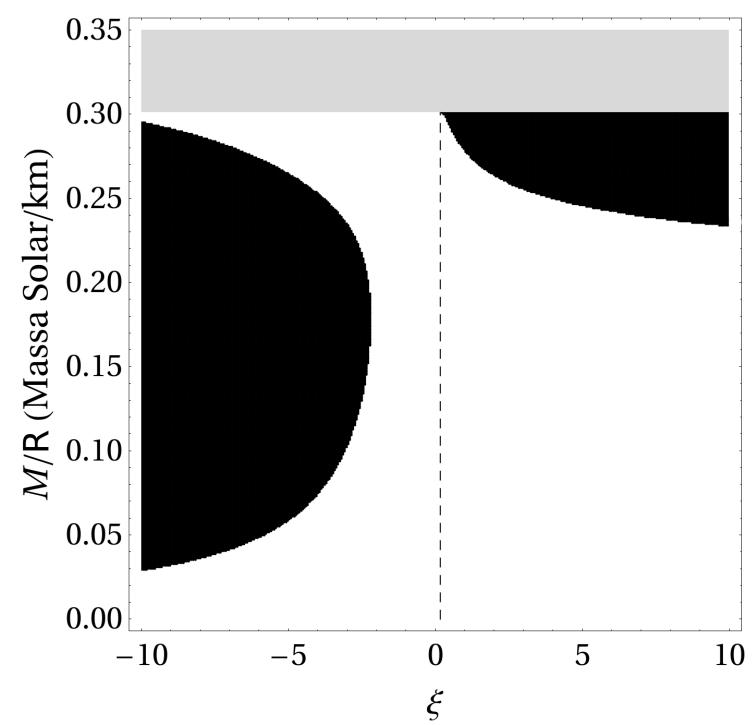

(a)

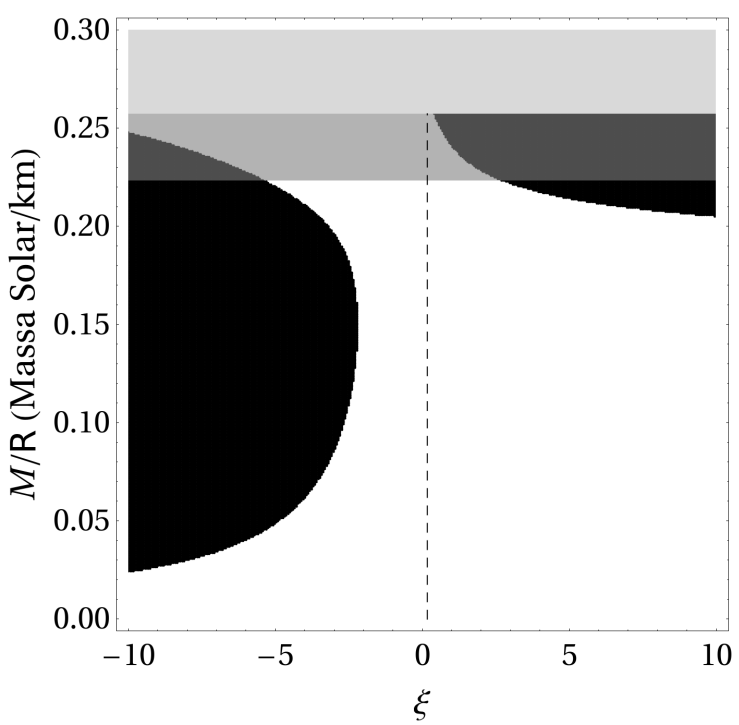

(b)

Figura 6.1 - Diagramas de "estados ligados" da Eq. 6.1.8 com autovalor negativo, $m=0$ e $l=0$. Cada ponto na região preta representa a existência de pelo menos uma solução da Eq. 6.1.8 com $\sigma$ negativo para um dado valor de $M / \mathrm{R}$ e $\xi$. Em ambos a linha vertical tracejada representa $\xi=1 / 6$. O diagrama (a) corresponde a uma estrela com densidade uniforme. A região cinza representa aqueles valores de $M / \mathrm{R}$ para os quais não há equilíbrio. O diagrama (b) foi construído a partir do perfil de densidade parabólico. Novamente a região cinza representa os valores de $M / \mathrm{R}$ para os quais não é possível o equilíbrio. Já a região demarcada por uma faixa cinza translúcida representa os valores de $M / \mathrm{R}$ para os quais configurações de equilíbrio são classicamente instáveis segundo equações de estado mais realistas.

É surpreendente que o mais simples dos sistemas, a saber, uma estrela de densidade 
uniforme $\rho(\chi)=\rho_{0}$, já seja capaz de detonar a explosão da densidade de energia do vácuo. Nesse caso, a existência desses "estados ligados" depende somente da razão entre a massa $M$ do objeto e de seu raio $\mathrm{R}$ e do parâmetro $\xi$. Esses resultados estão ilustrados na Fig. 6.1(a). No diagrama ali apresentado vê-se que para campos com $\xi>1 / 6$ ou $\xi \lesssim-2$ o efeito é disparado. Para esse caso ainda, o perfil radial do modo é apresentado nas Fig. 6.2(a) e Fig. 6.2(b) para duas combinações de valores para $M / \mathrm{R}$ e $\xi$. O perfis radiais do termo dominante da densidade de energia do vácuo associado à esses modos são mostrados, respectivamente, nas Fig. 6.3(a) e Fig. 6.3(b).

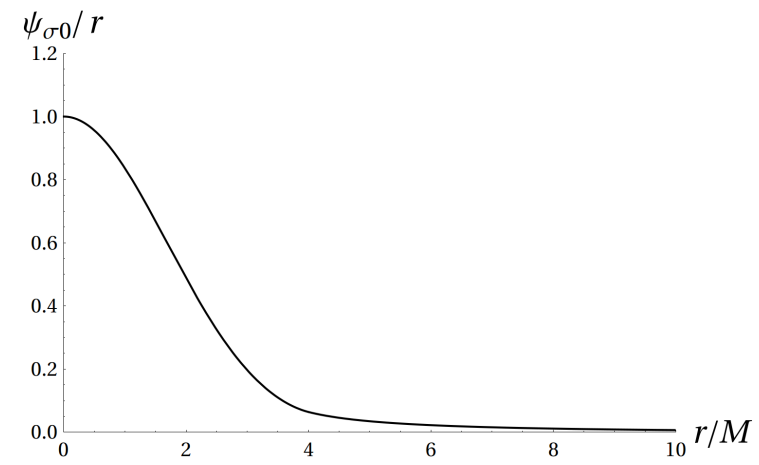

(a)

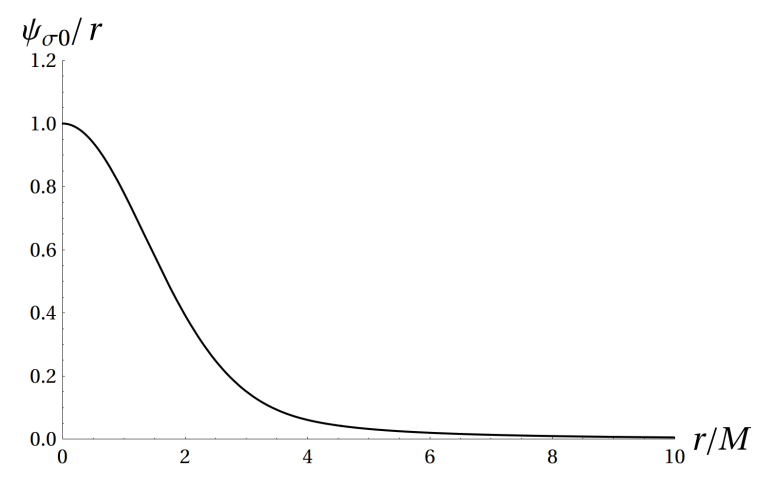

(c)

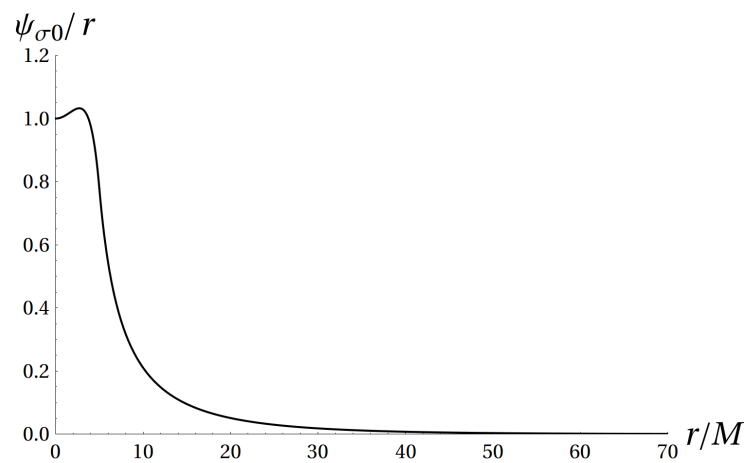

(b)

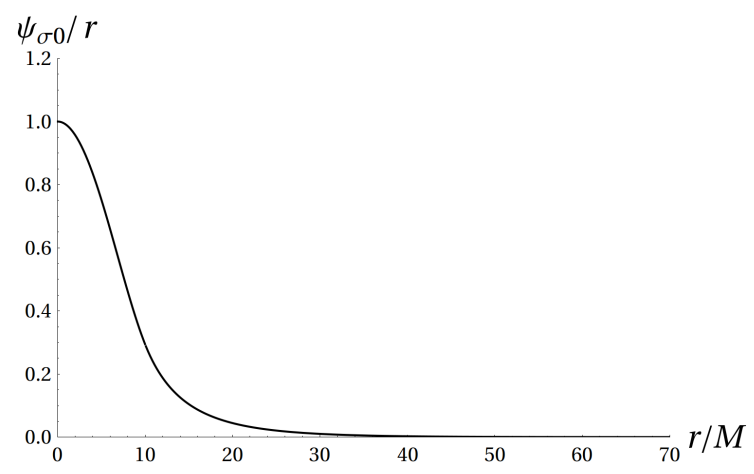

(d)

Figura 6.2 - Gráficos do perfil radial do modo exponencial com o menor valor para $\sigma$ em um espaço-tempo gerado por um corpo esfericamente simétrico e feito de fluido ideal. Em (a) e (b) o fluido tem densidade uniforme, enquanto que em (c) e (d) o perfil de densidade considerado é parabólico. A escala do eixo das abscissas é dada em $\mathrm{km} /$ Massa Solar. Todos os quatro casos acima a equação diferencial foi numericamente integrada a partir da condição de que $\psi_{\sigma 0}(0)=0$ e de que $\psi_{\sigma 0}^{\prime}(0)=1$. Os parâmetros utilizados para gerar as figuras são os seguintes: em (a) foi usado $\xi=5$ e $M / \mathrm{R}=0.25 \mathrm{~km} /$ Massa Solar, o que leva a $\sigma \approx-8.9 \times\left(M_{\odot} / M\right)^{2} \times 10^{8} \mathrm{~s}^{-2}$, em (b) foi usado $\xi=-3$ e $M / \mathrm{R}=0.2 \mathrm{~km} /$ Massa Solar, o que leva a $\sigma \approx-2.6 \times\left(M_{\odot} / M\right)^{2} \times 10^{8} \mathrm{~s}^{-2}$, em (c) foi usado $\xi=5$ e $M / \mathrm{R}=0.22 \mathrm{~km} /$ Massa Solar, o que leva a $\sigma \approx-1.2 \times\left(M_{\odot} / M\right)^{2} \times 10^{9} \mathrm{~s}^{-2}$ e em (d) foi usado $\xi=-5$ e $M / \mathrm{R}=0.1 \mathrm{~km} /$ Massa Solar, o que leva a $\sigma \approx-7.6 \times\left(M_{\odot} / M\right)^{2} \times 10^{8} \mathrm{~s}^{-2}$.

Apesar de a estrela com densidade uniforme ilustrar bem a afirmação de que espaçostempos bem-comportados são capazes de promover a amplificação da densidade de energia do vácuo, é necessário explorar perfis mais realistas se temos a esperança de que esse efeito 
tenha alguma aplicação observacional. Tanto quanto saibamos, não é conhecida uma equação de estado para matéria com densidades entre $10^{15}$ e $10^{17} \mathrm{~g} / \mathrm{cm}^{3}$. Entretanto, para estrelas de nêutrons com massas entre $1.5 M_{\odot}$ e $2.2 M_{\odot}, \rho=\rho_{\mathrm{c}}\left(1-r^{2} / \mathrm{R}^{2}\right)$ é uma boa aproximação para o perfil de densidade (45). Aqui $\rho_{\mathrm{c}}$, a densidade central, vale $15 M /\left(8 \pi \mathrm{R}^{3}\right)$. Assim como para o caso onde a densidade é uniforme, é possível resolver exatamente a equação de TOV para esse perfil parabólico mais realista (46). O diagrama de "estados ligados" com autovalores negativos para a Eq. 6.1.8 com o potencial efetivo construído a partir desse perfil parabólico está ilustrado na Fig. 6.1(b) e exemplos de perfis radiais do modo para diferentes valores dos parâmetros $M / \mathrm{R}$ e $\xi$ são apresentados nas Fig. 6.2(c) e Fig. 6.2(d). Para esses modos, os perfis radiais do termo dominante na densidade de energia do vácuo são apresentados, respectivamente, nas Fig. 6.3(c) e Fig. 6.3(d). Aqui novamente a existência de autovalores negativos somente depende da razão entre $M$ e R e do parâmetro $\xi$. Apesar da semelhança entre a Fig. 6.1(a) e a Fig. 6.1(b), nota-se que no caso mais realista o efeito é disparado para valores menores de $M /$ R. Em particular, vale frisar que valores de $M / \mathrm{R}$ para os quais equações de estado mais realista são causais - isto é, a velocidade do som não supera a velocidade da luz (45) - e representam configurações clássicas estáveis são capazes de despertar a energia do vácuo.

Outro aspecto interessante dos dois diagramas da Fig. 6.1 é a ausência de "estados ligados" para valores de $M / \mathrm{R}$ para os quais o espaço-tempo é estável por perturbações lineares da própria métrica. Para $\xi=0$, a condição de acoplamento mínimo, a equação de campo Eq. 3.1.2 simula perturbações da própria métrica sobre o espaço-tempo gerado pela presença da estrela. Logo, o fato de o efeito não ser detonado para valores dos parâmetros $M / \mathrm{R}$ fora da região cinza e $\xi=0$ é consistente com a estabilidade clássica do espaço-tempo de fundo.

Ainda vale mencionar que os termos dominantes da densidade de energia do vácuo apresentados nos gráficos da Fig. 6.3 ilustram bem a afirmação feita no Capítulo 5 de que quando o vácuo é despertado a densidade de energia fica exponencialmente positiva em algumas regiões da seção espacial, ao passo que em outras fica exponencialmente 


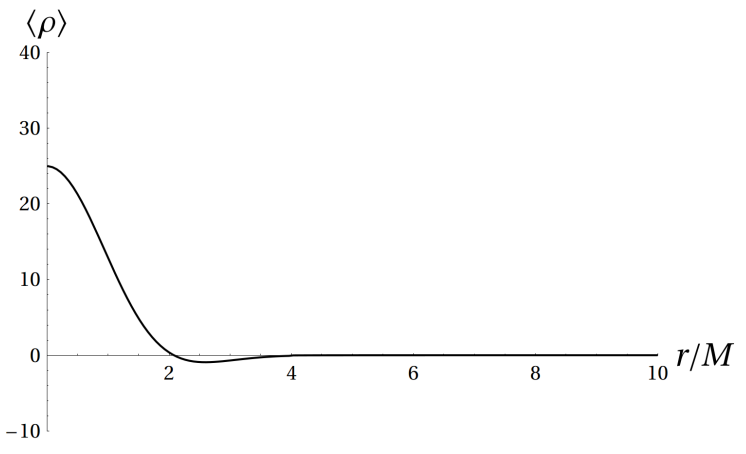

(a)

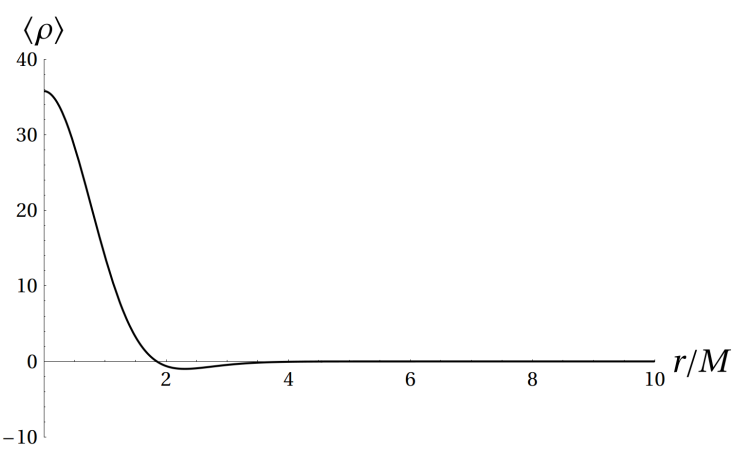

(c)

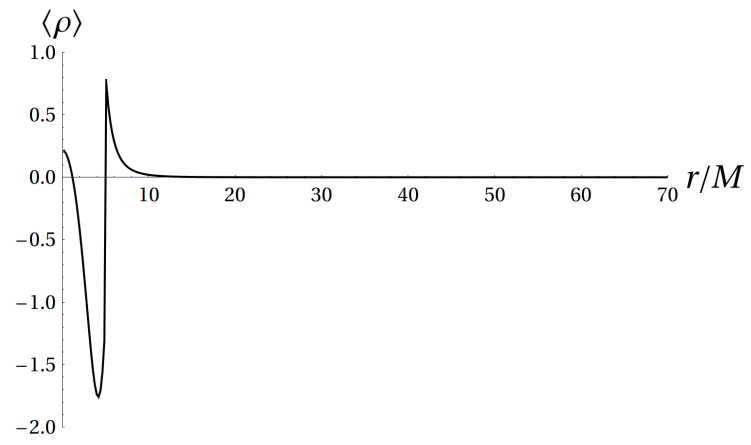

(b)

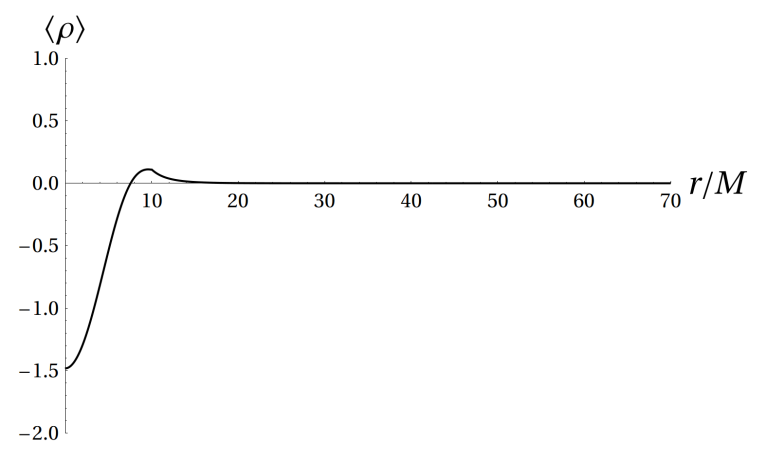

(d)

Figura 6.3 - Gráficos do perfil radial para a parcela dominante da densidade de energia de vácuo durante a fase instável. Os perfis apresentados nas figuras (a), (b), (c) e (d) estão associados, respectivamente, aos modos apresentados nas Fig. 6.2(a) Fig. 6.2(b) Fig. 6.2(c) e Fig. 6.2(d) A escala do eixo das abscissas é dada em km/Massa Solar enquanto que a escala do eixo das ordenadas é arbitrária. O fato dessa escala ser arbitrária não deve causar preocupação no leitor uma vez que o perfil apresentado cresce no tempo com um fator exponencial.

negativa. Como já apontado naquele capítulo, e ilustrado agora, a distribuição dessas regiões com densidade de energia positiva ou negativa depende da geometria de fundo aqui codificada pelo perfil de densidade da estrela - e do valor de $\xi$.

\subsection{Despertando a energia do vácuo no espaço-tempo de uma casca esférica estática}

Nesta seção vou dar atenção ao espaço-tempo gerado por uma casca esférica estática de massa $M$ e de raio $\mathrm{R}>2 M$. Em seu interior, por conta da simetria esférica, o espaçotempo é plano. Isso decorre do Teorema de Birkhoff e da condição de que as componentes da métrica não podem ser singulares no centro. Naturalmente, fora da casca o espaçotempo é o de Schwarzschild. Na região exterior usarei novamente a coordenada radial $\chi$ 
definida pelo vetor coordenado definido na Eq. (4.3.5). Já na região interna à casca o sistema de coordenadas é escolhido de modo tal que as componentes da métrica sejam funções contínuas em $r=\mathrm{R}$. O tensor métrico, então, pode ser colocado na seguinte forma:

$$
g_{a b}=-f\left[(d t)_{a}(d t)_{b}-(d \chi)_{a}(d \chi)_{b}\right]+r^{2}(\chi)\left[(d \theta)_{a}(d \theta)_{b}+\operatorname{sen}^{2} \theta(d \varphi)_{a}(d \varphi)_{b}\right]
$$

sendo que

$$
f(\chi)=1-\frac{2 M}{r(\chi)} \mathrm{H}\left(\chi-\chi_{0}\right)-\frac{2 M}{\mathrm{R}} \mathrm{H}\left(\chi_{0}-\chi\right) .
$$

Aqui $\mathrm{H}(x)$ denota a função de Heaviside ou função degrau e $\chi_{0}$ é tal que $r\left(\chi_{0}\right)=\mathrm{R}$. A relação entre as coordenadas radiais $r$ e $\chi$ pode ser obtida através da seguinte equação diferencial para a primeira:

$$
\frac{d r}{d \chi}(\chi)=\left[1-\frac{2 M}{r(\chi)}\right] \mathrm{H}\left(\chi-\chi_{0}\right)+\sqrt{1-\frac{2 M}{\mathrm{R}}} \mathrm{H}\left(\chi_{0}-\chi\right),
$$

assumindo que $r(\chi=0)=0$. Das expressões Eq. 6.2.2 e Eq. 6.2.3 fica claro que as componentes da métrica no sistema de coordenadas escolhido são funções contínuas mas com primeira derivada com relação a $\chi$ descontínua. Portanto, quantidades geométricas, como o escalar de curvatura, onde comparecem segundas derivadas da métrica, são singulares em $r=\mathrm{R}$. Por conta disso, essas quantidades divergentes devem ser interpretadas no sentido de distribuição $1^{1}$

Uma vez que estou interessado em soluções da Eq. (3.1.2), faz-se necessária a expressão do escalar de curvatura nessas coordenadas. De sua definição, Eq. 2.1.4 e da Eq. 6.2.1) para a métrica do espaço-tempo vem que

$$
R=-\frac{1}{f} \frac{d}{d \chi}\left(\frac{1}{f} \frac{d f}{d \chi}\right)-\frac{4}{r f} \frac{d^{2} r}{d \chi^{2}}+\frac{2}{r^{2}}\left[1-\frac{1}{f}\left(\frac{d r}{d \chi}\right)^{2}\right] .
$$

Substituindo na forma acima para o escalar de curvatura Eq. 6.2.2 e Eq. 6.2.3), temos

$$
R=-\frac{4}{\mathrm{R}}\left(1-\frac{2 M}{\mathrm{R}}\right)^{-1}\left(1-\frac{3}{2} \frac{M}{\mathrm{R}}-\sqrt{1-\frac{2 M}{\mathrm{R}}}\right) \delta\left(\chi-\chi_{0}\right),
$$

\footnotetext{
${ }^{1}$ Para uma exposição sobre o problema da colagem de espaços-tempos através de superfícies veja, e.g., a referência $(47)$.
} 
onde $\delta(x)$ denota a distribuição delta.

Por conta das simetrias assumidas para o espaço-tempo de fundo, posso aqui aplicar à solução da Eq. (3.1.2) as mesmas decomposições que levaram à Eq. (6.1.8). Novamente o potencial efetivo tem a forma da Eq. (6.1.9), a qual implica para um campo sem massa, no caso do espaço-tempo gerado pela casca esférica, que

$$
\begin{aligned}
V_{\mathrm{ef}}^{(l)}= & \frac{l(l+1)}{r^{2}}\left[1-\frac{2 M}{r} \mathrm{H}\left(\chi-\chi_{0}\right)-\frac{2 M}{r_{0}} \mathrm{H}\left(\chi_{0}-\chi\right)\right] \\
& +\frac{2 M}{r^{3}}\left(1-\frac{2 M}{r}\right) \mathrm{H}\left(\chi-\chi_{0}\right)+\gamma \delta\left(\chi-\chi_{0}\right),
\end{aligned}
$$

sendo que

$$
\gamma:=\frac{1-4 \xi}{\mathrm{R}}-(1-3 \xi) \frac{2 M}{\mathrm{R}^{2}}-\frac{1-4 \xi}{\mathrm{R}} \sqrt{1-\frac{2 M}{\mathrm{R}}}
$$

Desejo agora mostrar que a Eq. (6.1.8) com o potencial efetivo dado na Eq. (6.2.6) admite pelo menos uma solução para $\sigma<0$ tal que

$$
\begin{gathered}
\lim _{\chi \rightarrow 0}\left|\frac{\psi_{\sigma l}(\chi)}{r(\chi)}\right|<+\infty, \\
\lim _{\chi \rightarrow+\infty} \psi_{\sigma l}(\chi)=0, \\
\lim _{\chi \rightarrow \chi_{0}^{-}} \psi_{\sigma l}(\chi)=\lim _{\chi \rightarrow \chi_{0}^{+}} \psi_{\sigma l}(\chi)
\end{gathered}
$$

e

$$
\lim _{\chi \rightarrow \chi_{0}^{+}} \frac{d}{d \chi} \psi_{\sigma l}-\lim _{\chi \rightarrow \chi_{0}^{-}} \frac{d}{d \chi} \psi_{\sigma l}=\gamma \psi_{\sigma l}\left(\chi_{0}\right)
$$

A estratégia para abordar esse problema será a mesma que foi empregada na seção anterior. Aqui a obrigação de a solução divergir com o sinal trocado em relação àquele sobre a casca implicará em uma desigualdade a ser satisfeita e que dependerá de R e $\xi$. Para implementar essa abordagem é necessário primeiro descobrir o comportamento da parte radial dos modos para $\sigma=0$ quando $\chi \rightarrow+\infty$, o que só é possível resolvendo sua equação.

Para o caso da casca é possível encontrar a solução da Eq. 6.1.8 em termos de funções conhecidas. De fato. Usando explicitamente a forma do potencial efetivo $V_{\mathrm{ef}}^{(l)}(\chi)$ para o 
caso da casca esférica naquela equação, temos

$$
\left\{\begin{aligned}
-\frac{d^{2}}{d \chi^{2}} \psi_{\sigma l}+\left(1-\frac{2 M}{\mathrm{R}}\right) \frac{l(l+1)}{r^{2}} \psi_{\sigma l} & =\sigma \psi_{\sigma l}, \quad 0<\chi<\chi_{0}, \\
-\frac{d^{2}}{d \chi^{2}} \psi_{\sigma l}+\left(1-\frac{2 M}{r}\right)\left[\frac{l(l+1)}{r^{2}}+\frac{2 M}{r^{3}}\right] \psi_{\sigma l} & =\sigma \psi_{\sigma l}, \quad \chi_{0}<\chi<+\infty .
\end{aligned}\right.
$$

Para $\sigma=0$ a equação diferencial acima tem a seguinte solução geral:

$$
\psi_{0 l}= \begin{cases}A_{1}^{(l)} r^{l+1}+A_{2}^{(l)} r^{-l}, & 0<r<\mathrm{R}, \\ B_{1}^{(l)} Q_{l}\left(\frac{r}{M}-1\right) r+B_{2}^{(l)} P_{l}\left(\frac{r}{M}-1\right) r, & \mathrm{R}<r<+\infty,\end{cases}
$$

onde $P_{l}(z)$ e $Q_{l}(z)$ são, respectivamente, as funções associadas de Legendre de primeira e segunda espécie de ordem zero e grau $\nu=l$, como definido na referência (48). O fato de que $\sigma=0$ faz com que seja mais conveniente utilizar a variável $r$ ao invés de $\chi$. Dado que na origem o modo do campo deve ser regular, é necessário impor que $A_{2}^{(l)}=0$. Por conveniência, vou escolher $A_{1}^{(l)}=1 / \mathrm{R}^{l}$. Isso, através das condições sobre $\psi_{\sigma l}$ e sua derivada primeira na casca, fixa as constantes $B_{1}^{(l)}$ e $B_{2}^{(l)}$. Por fim, tem-se

$$
\psi_{0 l}= \begin{cases}r^{l+1} / \mathrm{R}^{l}, & 0<r<\mathrm{R}, \\ \frac{\left\{\left[1-\frac{2 M}{\mathrm{R}}+\frac{l M}{\mathrm{R}}\left(\frac{\mathrm{R}}{M}-1\right)-\sqrt{1-\frac{2 M}{\mathrm{R}}}(l+1)-\gamma \mathrm{R}\right] P_{l}\left(\frac{\mathrm{R}}{M}-1\right)-\frac{l M}{\mathrm{R}} P_{l-1}\left(\frac{\mathrm{R}}{M}-1\right)\right\} Q_{l}\left(\frac{r}{M}-1\right) r}{\frac{l M M}{\mathrm{R}}\left[P_{l}\left(\frac{\mathrm{R}}{M}-1\right) Q_{l-1}\left(\frac{\mathrm{R}}{M}-1\right)-P_{l-1}\left(\frac{\mathrm{R}}{M}-1\right) Q_{l}\left(\frac{\mathrm{R}}{M}-1\right)\right]} & \\ -\frac{\left\{\left[1-\frac{2 M}{\mathrm{R}}+\frac{l M}{\mathrm{R}}\left(\frac{\mathrm{R}}{M}-1\right)-\sqrt{1-\frac{2 M}{\mathrm{R}}}(l+1)-\gamma \mathrm{R}\right] Q_{l}\left(\frac{\mathrm{R}}{M}-1\right)-\frac{l M}{\mathrm{R}} Q_{l-1}\left(\frac{\mathrm{R}}{M}-1\right)\right\} P_{l}\left(\frac{r}{M}-1\right) r}{\frac{\mathrm{R}}{\mathrm{R}}\left[P_{l}\left(\frac{\mathrm{R}}{M}-1\right) Q_{l-1}\left(\frac{\mathrm{R}}{M}-1\right)-P_{l-1}\left(\frac{\mathrm{R}}{M}-1\right) Q_{l}\left(\frac{\mathrm{R}}{M}-1\right)\right]}, & \mathrm{R} \leq r<+\infty .\end{cases}
$$

Note que a solução acima aparentemente não estaria definida para $l=0$ no intervalo $[\mathrm{R},+\infty[$. Esse, entretanto, não é o caso. Através das relações 8.2.1 e 8.2.2 da referência (48) entre funções associadas de Legendre de grau positivo e negativo, é possível mostrar que os coeficientes $B_{1}^{(\nu)}$ e $B_{2}^{(\nu)}$ têm limite bem definido quando $\nu \rightarrow 0$. Desse procedimento decorre que

$$
\psi_{00}= \begin{cases}r, & 0<r<\mathrm{R}, \\ \frac{\mathrm{R}}{2 M}\left(1-\frac{2 M}{\mathrm{R}}-\sqrt{1-\frac{2 M}{\mathrm{R}}}-\gamma \mathrm{R}\right) \ln \left(\frac{1-\frac{2 M}{\mathrm{R}}}{1-\frac{2 M}{r}}\right) r+r, & \mathrm{R} \leq r<+\infty .\end{cases}
$$

Voltemos, agora, à abordagem proposta para a busca por soluções da Eq. (6.1.8) com $\sigma<0$. Da série assintótica para as funções associadas de Legendre é fácil ver que $\lim _{r \rightarrow+\infty} Q_{l}\left(\frac{r}{M}-1\right) r=0$ e que $\lim _{r \rightarrow+\infty} P_{l}\left(\frac{r}{M}-1\right) r=+\infty$. Uma vez que $\psi_{0 l}(\mathrm{R})=$ 
$\mathrm{R}>0$, então há pelo menos uma solução com $\sigma<0$ se a condição

$$
\frac{\left[1-\frac{2 M}{\mathrm{R}}+\frac{l M}{\mathrm{R}}\left(\frac{\mathrm{R}}{M}-1\right)-\sqrt{1-\frac{2 M}{\mathrm{R}}}(l+1)-\gamma \mathrm{R}\right] Q_{l}\left(\frac{\mathrm{R}}{M}-1\right)-\frac{l M}{\mathrm{R}} Q_{l-1}\left(\frac{\mathrm{R}}{M}-1\right)}{\frac{l M}{\mathrm{R}}\left[P_{l}\left(\frac{\mathrm{R}}{M}-1\right) Q_{l-1}\left(\frac{\mathrm{R}}{M}-1\right)-P_{l-1}\left(\frac{\mathrm{R}}{M}-1\right) Q_{l}\left(\frac{\mathrm{R}}{M}-1\right)\right]}>0
$$

é satisfeita. Em particular, para $l=0$ essa condição toma a forma

$$
\frac{\mathrm{R}}{2 M}\left[\gamma \mathrm{R}+\sqrt{1-\frac{2 M}{\mathrm{R}}}-\left(1-\frac{2 M}{\mathrm{R}}\right)\right] \ln \left(1-\frac{2 M}{\mathrm{R}}\right)-1>0 .
$$

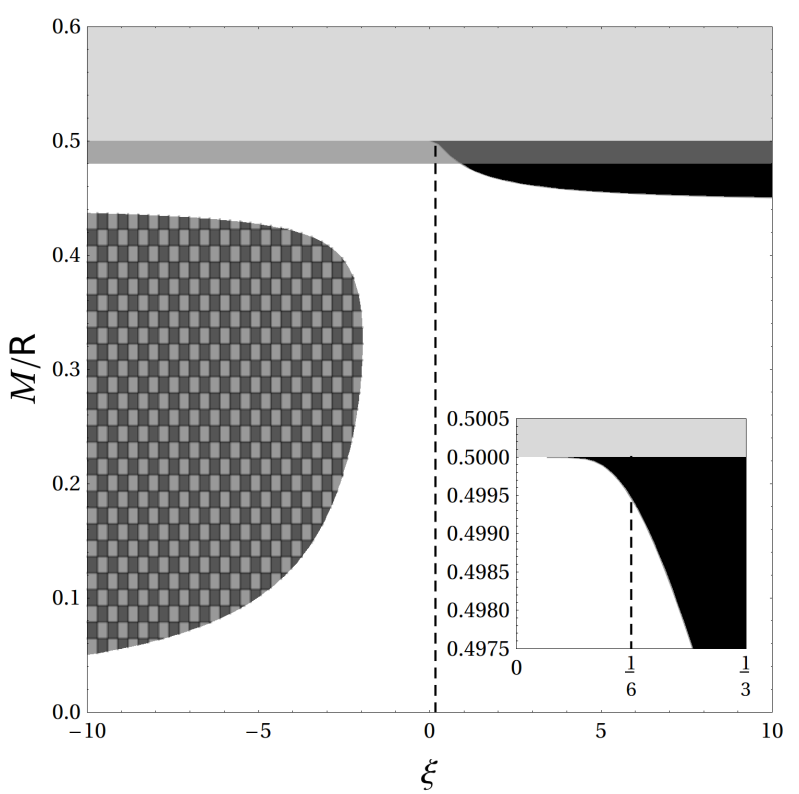

(a)

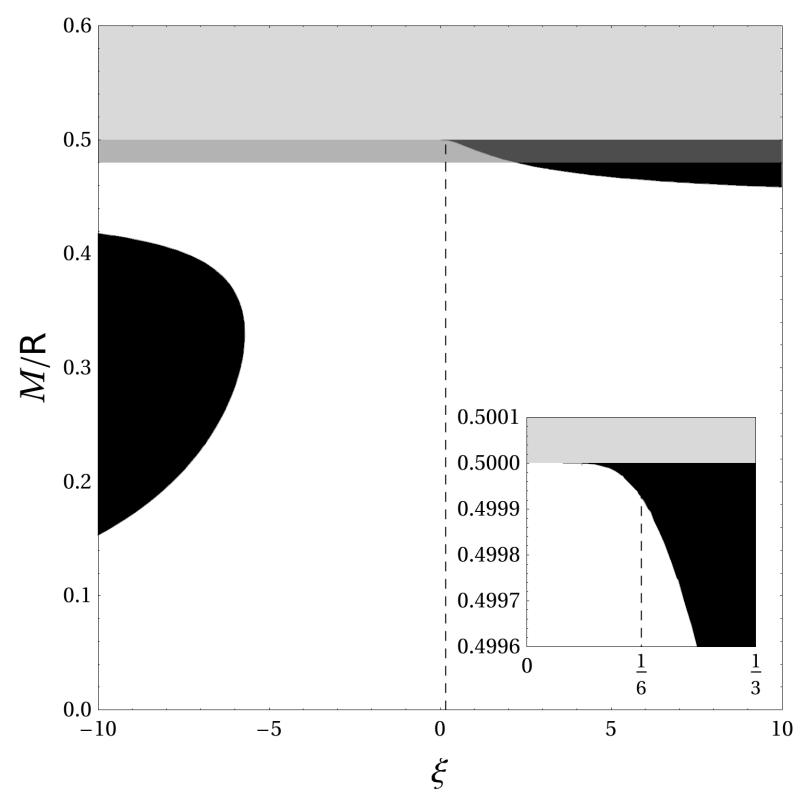

(b)

Figura 6.4 - Diagramas de "estados ligados" da Eq. 6.1 .8 com autovalor negativo, para um campo sem massa e $l=0$ em (a) e $l=1$ em (b). Cada ponto na região preta e quadriculada em (a) e preta em (b) representa a existência de pelo menos uma solução da Eq. (6.1.8) com $\sigma$ negativo para um dado valor de $M / \mathrm{R}$ e $\xi$. Neles a linha vertical tracejada representa $\xi=1 / 6$. A região cinza-clara representa aqueles valores de $M / \mathrm{R}$ para os quais a casca viola o limite de Schwarzschild enquanto que a definida pela faixa cinza translúcida representa as configurações da casca que violam a condição de energia dominante. Em cada um dos diagramas, no canto inferior direito, está destacada a região em torno de $\xi=1 / 6$, valor este para $\xi$ novamente identificado por uma linha pontilhada. Os eixos têm o mesmo rótulo que os do diagrama maior. Em (a) a região preta denota os valores dos parâmetros para os quais a contribuição do efeito à densidade de energia da casca é positiva, enquanto na região quadriculada essa contribuição é negativa.

Nos diagramas Fig. 6.4 e Fig. 6.5 está ilustrado a região de valores de $M / \mathrm{R}$ e $\xi$ na qual as desigualdades acima são satisfeitas para diferentes valores de $l$. Apesar de qualitativamente semelhantes aos diagramas na Fig. 6.1, o caso da casca esférica guarda uma característica especial. Diferentemente do que foi encontrado no caso da estrela, o espaço-tempo gerado pela casca admite soluções para a equação de campo que são exponencialmente divergentes quando $\xi=1 / 6$, mesmo para $l \neq 0$. Para esse caso, 


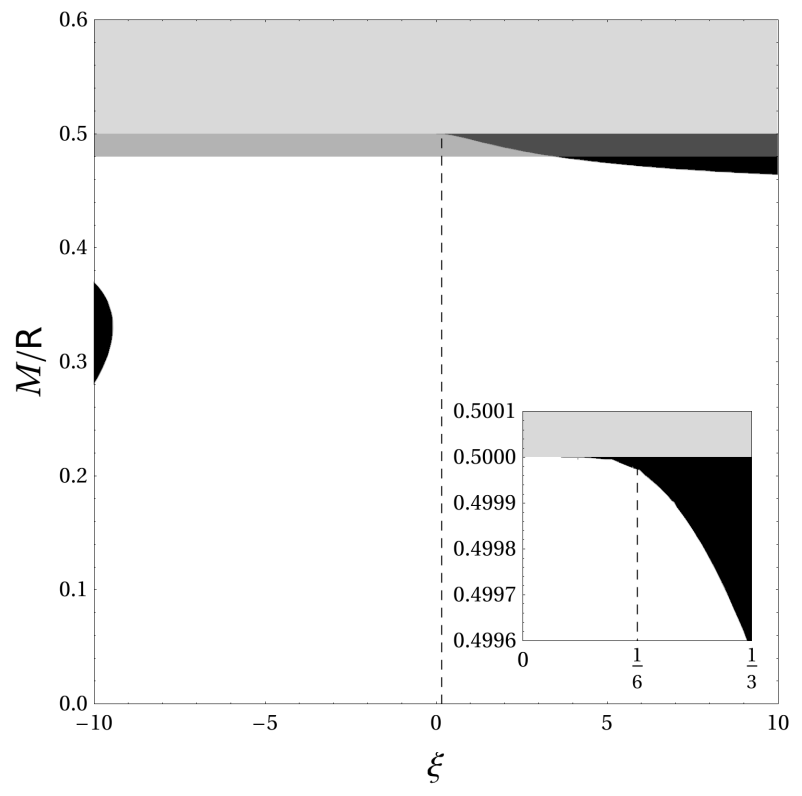

(a)

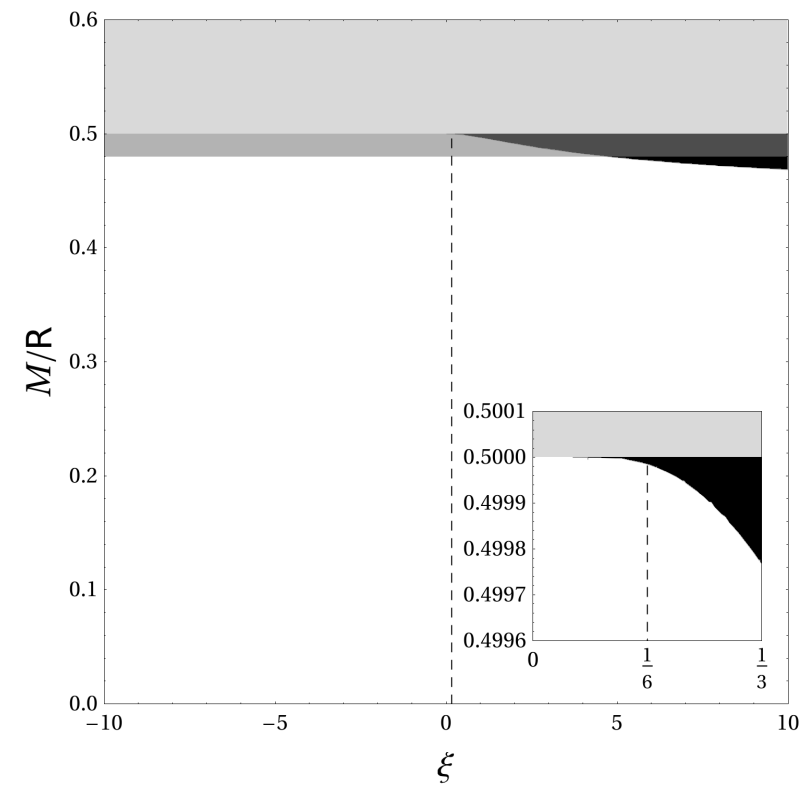

(b)

Figura 6.5 - O mesmo tipo de diagrama da Fig. 6.4 Aqui $l=2 \mathrm{em}$ (a) e $l=3 \mathrm{em}(\mathrm{b})$.

entretanto, a razão massa-raio da casca é muito próxima àquela do limite de Schwarzschild e viola a condição de energia dominante. Esse comportamento das soluções na região em torno de $\xi=1 / 6$ pode ser apreciado no canto inferior direito dos diagramas Fig. 6.4 e Fig. 6.5.

Considere agora um cenário semelhante ao da formação da estrela, onde a casca somente desencadeia o efeito assintoticamente no futuro. O valor esperado do tensor energia-momentum do campo pode ser colocado na seguinte forma:

$$
\left\langle 0_{\text {in }}\left|\hat{T}_{a b}\right| 0_{\text {in }}\right\rangle=\left\langle\hat{T}_{a b}^{(+)}\right\rangle \mathrm{H}(\ell)+\left\langle\hat{T}_{a b}^{(-)}\right\rangle \mathrm{H}(-\ell)+\left\langle\hat{T}_{a b}^{(S)}\right\rangle,
$$

sendo que \pm indica, respectivamente, se as componentes do tensor são calculadas com quantidades definidas fora ou dentro da casca e $\left\langle\hat{T}_{a b}^{(S)}\right\rangle$ denota a parte do tensor definido sobre a casca. Aqui $\ell$ é um escalar definido através das linhas integrais do campo vetorial ortogonal à casca e é tal que se anula sobre ela. Por conta da descontinuidade da derivada dos modos, a parte do tensor energia-momentum definido sobre a casca diverge, mas pode ser escrito como

$$
\left\langle\hat{T}_{a b}^{(S)}\right\rangle=\left\langle\hat{S}_{a b}\right\rangle \delta(\ell)
$$


Logo, esse termo contribui para o tensor energia-momentum da casca, que deve divergir da mesma forma a fim de gerar a métrica Eq. 6.2.1. Assintoticamente no futuro, podemos calcular a forma de algumas componentes de $\left\langle 0_{\text {in }}\left|\hat{T}_{a b}\right| 0_{\text {in }}\right\rangle$ em termos da parcela dominante. Para a densidade de energia, por exemplo, vem que

$$
\begin{aligned}
\left\langle\hat{\rho}^{( \pm)}\right\rangle:=n^{a} n^{b}\left\langle\hat{T}_{a b}^{( \pm)}\right\rangle \stackrel{\text { futuro }}{\sim} \kappa \frac{\bar{\Omega}}{8 \pi} e^{2 \bar{\Omega} t}\{ & \frac{1-4 \xi}{4 f r^{2}} \frac{d}{d \chi}\left[r^{2} \frac{d}{d \chi}\left(\frac{\psi_{\bar{\Omega} 0}}{\bar{\Omega} r}\right)^{2}\right] \\
& \left.+\frac{1}{2} \frac{\xi}{f \bar{\Omega}^{2} r^{2}} \frac{d}{d \chi}\left(\frac{\psi_{\bar{\Omega} 0}^{2}}{f} \frac{d f}{d \chi}\right)\right\},
\end{aligned}
$$

e

$$
\begin{aligned}
\left\langle\hat{\rho}^{(S)}\right\rangle:=n^{a} n^{b}\left\langle\hat{T}_{a b}^{(S)}\right\rangle \stackrel{\text { futuro }}{\sim} \kappa \frac{\bar{\Omega}}{8 \pi} e^{2 \bar{\Omega} t}\left\{\frac{1-4 \xi}{2}\left[\gamma+\frac{1}{\mathrm{R}}\left(\sqrt{1-\frac{2 M}{\mathrm{R}}}-\left(1-\frac{2 M}{\mathrm{R}}\right)\right)\right]\right. \\
\left.+\frac{\xi}{2} \frac{2 M}{\mathrm{R}^{2}}\right\} \frac{\psi_{\bar{\Omega} 0}^{2}}{f \bar{\Omega}^{2} r^{2}} \delta\left(\chi-\chi_{0}\right) .
\end{aligned}
$$

Na Fig. 6.4(a) a região preta do diagrama mostra os valores de $M / \mathrm{R}$ e $\xi$ para os quais $\left\langle\hat{\rho}^{(S)}\right\rangle>0$, enquanto que na região quadriculada $\left\langle\hat{\rho}^{(S)}\right\rangle<0$.

Apesar de não levarmos em conta a retroação do campo quântico sobre o espaçotempo de fundo, podemos especular sobre o destino do sistema através dos sinais do valor esperado da densidade de energia do vácuo. Quando positiva, essa contribuição irá aumentar a energia da casca possivelmente até desestabilizá-la. A partir desse ponto a casca pode colapsar, escondendo dentro do horizonte de eventos parte da energia do vácuo acumulada durante a atuação do efeito, o que pararia o mecanismo. A energia do vácuo restante poderia, então, ser liberada através da criação de partículas do próprio campo. Por outro lado, quando negativa, essa contribuição poderia ajudar a estabilizar o sistema. Entretanto, pode ser mostrado a partir da Eq. 6.2.17 que a densidade de energia acumulada no interior da casca é sempre positiva quando $\xi<1 / 4$. Logo, se não for possível estabilizar o sistema até o ponto em que a energia de vácuo acumulada dentro da casca atinge o limite de Schwarzschild, poderíamos ter a formação de um buraco negro com raio da mesma ordem do raio da casca. Tal desfecho poderia levar ao colapso de todo o sistema, o que possivelmente cessaria o mecanismo de despertar do vácuo. 


\section{Capítulo 7}

\section{Espaço de estados do campo na presença da instabilidade}

1 os capítulos precedentes foi mostrado que em situações bastante razoáveis o vácuo da geometria de fundo. Até este ponto, contudo, pouco foi dito a respeito do espaço de estados do campo, $\mathscr{F}_{\mathrm{s}}\left(\mathscr{H}_{\text {out }}\right)$, quando o efeito é disparado. A construção, segundo uma receita análoga a apresentada na Seção 3.2, e a interpretação física desse espaço são questões que acredito serem bastante interessantes, particularmente pelo fato de que é pouco usual o estudo da quantização de campos quando da presença de modos que crescem exponencialmente.

Para abordar essa questão, na Seção 7.1 apresento uma prescrição para construção do espaço de Hilbert de uma partícula quando da presença da instabilidade. O espaço de Hilbert de uma partícula resultante, aqui definido a partir de um produto interno definido sobre o espaço de soluções complexas $\mathscr{S}^{\mathbb{C}}$, já foi obtido através dos projetores espectrais do operador $\Delta^{\text {out }}$ os conjuntos de modos $\left\{v_{l}\right\}_{l \in \mathcal{O}}$ e $\left\{w_{n}\right\}_{n \in \mathcal{O}^{\prime}}$ - no capítulo 5 . Tanto quanto eu saiba, a construção que será a presentada na Seção 7.1 é original. Ademais, de maneira a obter uma interpretação física mais clara do espaço $\mathscr{F}_{\mathrm{s}}\left(\mathscr{H}_{\text {out }}\right)$, na Seção 7.2 exploro a relação que existe entre os graus de liberdade do campo quântico descritos pelos 
modos exponenciais e partículas quânticas não-relativística submetidas a uma barreira de potencial parabólica.

\subsection{Construção do espaço de estados}

Da forma com que foi apresentado no Capítulo 5, o Efeito de Despertar do Vácuo é produto de um problema dinâmico de um sistema cujo estado inicial é bem-comportado. O espaço de estados inicial, $\mathscr{F}_{\mathrm{s}}\left(\mathscr{H}_{\text {in }}\right)$, é construído segundo o algoritmo apresentado na Seção 3.2. Lá o ponto-chave da receita é o uso da conservação da energia para induzir um mapeamento natural entre o espaço de soluções reais da equação de campo, $\mathscr{S}$, e o espaço de Hilbert de uma partícula $\mathscr{H}_{\text {in }}$. Isso só é possível, todavia, devido ao fato de que a energia dos perfis de campo, como definida por observadores seguindo as linhas integrais do campo de Killing tipo tempo $\varkappa^{a}$, é estritamente positiva. Vejamos o que acontece com a energia do campo quando da presença da instabilidade estudada nesta tese.

Vou começar reescrevendo de maneira mais adequada a forma sesquilinear definida na Eq. 3.2.5. Quando o espaço-tempo é estático é possível mostrar que para $\psi_{1}, \psi_{2} \in \mathscr{S}^{\mathbb{C}}$

$$
\left\langle\psi_{1}, \psi_{2}\right\rangle=\frac{1}{2} \int_{\Sigma_{t}} \frac{d \Sigma}{\|\varkappa\|}\left(\bar{p}_{1} p_{2}+\bar{\phi}_{1} \Delta \phi_{2}\right)
$$

onde $p:=\varkappa^{a} \nabla_{a} \phi$ e

$$
\Delta:=-\|\varkappa\| D^{a}\left(\|\varkappa\| D_{a} \cdot\right)+\|\varkappa\|^{2}\left(m^{2}+\xi R\right) \cdot
$$

o operador diferencial que comparece no lado esquerdo da Eq. 4.2.16. Considere, então, uma solução complexa da equação de campo dada por

$$
\phi=\sum_{n \in \mathcal{O}^{\prime}}\left(c_{n} w_{n}+\bar{d}_{n} \bar{w}_{n}\right)
$$

onde os $w_{n}$ têm a forma dada pela Eq. (5.1.22). Substituindo a solução $\phi$, Eq. (7.1.3), na forma sesquilinear Eq. (7.1.1) segue

$$
\langle\psi, \psi\rangle=-\frac{\sqrt{3}}{2} \sum_{n \in \mathcal{O}^{\prime}} \Omega_{n}\left(\left|c_{n}\right|^{2}+\left|d_{n}\right|^{2}\right)-\sum_{n \in \mathcal{O}^{\prime}} \Omega_{n}\left(c_{n} d_{n}+\bar{c}_{n} \bar{d}_{n}\right) .
$$


Daí vê-se que, quando da presença dos modos $w_{n}$, há elementos de $\mathscr{S}^{\mathbb{C}}$ para os quais a forma sesquilinear da Eq. (7.1.1) não atribui um número positivo, o que implica que ela não define um produto interno no espaço de soluções complexas do campo. Portanto, o algoritmo apresentado na Seção 3.2, que parte da forma sesquilinear Eq. (7.1.1), não é adequado para definir o espaço de Hilbert de uma partícula se o espectro de $\Delta$ tem uma parte negativa.

Isso pode ser contornado provendo uma outra forma sesquilinear sobre $\mathscr{S}^{\mathbb{C}}$ que de fato defina um produto interno. Sabemos, por conta da forma dos modos $v_{l}$, que qualquer que seja essa nova forma ela deve coincidir com a forma sesquilinear dada na Eq. 7.1.1 quando a solução pode ser escrita somente em termos de modos que oscilam no tempo. Por outro lado, se visto como um operador cujo domínio está contido no espaço das funções com suporte em uma hiperfície $\Sigma_{t}$ que são de quadrado integrável na media $\frac{d \Sigma}{\|\iota\|}$, $L^{2}\left(\Sigma_{t}, \frac{d \Sigma}{\|\varkappa\|}\right), \Delta$ é essencialmente auto-adjunto em $C_{0}^{\infty}\left(\Sigma_{t}\right)$. (Para a prova dessa afirmação veja as referências $(49,50)$.) Isso significa que existe uma única extensão auto-adjunta desse operador, o seu fecho, denotada por $\bar{\Delta}$. Pelo Teorema da Decomposição Polar (veja, e.g., o Teorema VIII.32 da referência (30)), associado a $\bar{\Delta}$ existe um operador autoadjunto e positivo, $|\bar{\Delta}|$, cujo domínio é idêntico ao domínio de $\bar{\Delta}$. O operador $|\bar{\Delta}|$ pode ser construído via o Teorema Espectral fazendo $|\bar{\Delta}|=\sqrt{\bar{\Delta}^{2}}$. Com isso em mente, considere a forma sesquilinear $\langle\cdot, \cdot\rangle_{|\bar{\Delta}|}: \mathscr{S}^{\mathbb{C}} \times \mathscr{S}^{\mathbb{C}} \rightarrow \mathbb{C}$ definida por

$$
\left\langle\psi_{1}, \psi_{2}\right\rangle_{|\bar{\Delta}|}:=\frac{1}{2} \int_{\Sigma_{t}} \frac{d \Sigma}{\|\varkappa\|}\left(\bar{p}_{1} p_{2}+\bar{\phi}_{1}|\bar{\Delta}| \phi_{2}\right)
$$

Essa forma sesquilinear é evidentemente positiva quando $\psi_{1}=\psi_{2}$. Logo, mesmo na presença da instabilidade, a Eq. (7.1.5) define um produto interno sobre $\mathscr{S}^{\mathbb{C}}$. Esse produto interno guarda uma característica interessante. Uma vez que ele não pode ser derivado a partir da conservação do tensor energia-momentum associado ao campo, o valor de $\left\langle\psi_{1}, \psi_{2}\right\rangle_{|\bar{\Delta}|}$ dependerá, em geral, da hiperfície $\Sigma_{t}$ escolhida para o cálculo da integral que comparece no lado direito da definição da Eq. 7.1.5 mesmo quando o espaço-tempo de fundo é estático. Desse modo, o vácuo da representação construída a partir desse produto interno não será estacionário. Note ainda que essa não-estacionariedade do vácuo está 
diretamente ligada ao setor correspondente aos modos $w_{n}$, haja visto que a atuação de $|\bar{\Delta}|$ sobre o subespaço associado à parte positiva do espectro de $\bar{\Delta}$ é idêntica à do próprio $\bar{\Delta}$.

A discussão sobre a quantização de campos livres em espaços-tempos globalmente hiperbólicos da Seção 3.1 está estruturada sobre a hipótese da existência de um produto interno $\mu$ sobre o espaço $\mathscr{S}$ satisfazendo a condição dada na Eq. (3.1.13). Na referência (36) P. Chmielowski mostra que dado um produto interno sobre $\mathscr{S}^{\mathbb{C}}$ satisfazendo para $\operatorname{algum} C>0$

$$
C\left\|\psi_{1}\right\|\left\|\psi_{2}\right\| \geq \frac{1}{2}\left|\Omega\left(\psi_{1}, \psi_{2}\right)\right|
$$

para todo $\psi_{1}, \psi_{2} \in \mathscr{S}^{\mathbb{C}}$ existe, então, um produto interno $\mu$ sobre $\mathscr{S}$ tal que a condição da Eq. 3.1.13 é satisfeita. É possível provar que o produto interno definido pela forma da Eq. 7.1.5 satisfaz a desigualdade Eq. 7.1.6), desde que para todo $\lambda \in \sigma(\bar{\Delta})$, o espectro de $\bar{\Delta}$, exista uma constante $M>0$ tal que $|\lambda|>M$ e que existam constantes $\varepsilon_{1}, \varepsilon_{2}>0$ tais que em $\Sigma_{t}$ valha $\varepsilon_{1} \leq\|\varkappa\| \leq \varepsilon_{2}$. Relegarei essa prova ao Apêndice A.

Seguindo a prova de Chmielowski (veja também o final da Seção 4.3 da referência (7)) é possível ainda construir o mapeamento entre $\mathscr{S}$ e o espaço de Hilbert de uma partícula desejado. Vejamos. Em primeiro lugar, considere o espaço $\mathscr{E}$ obtido através do completamento de $\mathscr{S}^{\mathbb{C}}$ pelo produto interno definido na Eq. 7.1.5). Note, então, que devido à desigualdade Eq. 7.1.6 existe um operador $\hat{A}: \mathscr{E}^{\prime} \rightarrow \mathscr{E}^{\prime}$ limitado tal que

$$
\left\langle\psi_{1}, \hat{A} \psi_{2}\right\rangle_{|\bar{\Delta}|}=i \Omega\left(\bar{\psi}_{1}, \psi_{2}\right)
$$

Ademais, a anti-simetria de $\Omega$ implica que $\hat{A}$ é auto-adjunto. Em seguida, define-se o produto interno $\mu_{|\bar{\Delta}|}(\cdot, \cdot): \mathscr{S} \times \mathscr{S} \rightarrow \mathbb{R}$ como

$$
\mu_{|\bar{\Delta}|}\left(\psi_{1}, \psi_{2}\right):=\frac{1}{2}\left\langle\psi_{1},|\hat{A}| \psi_{2}\right\rangle_{|\bar{\Delta}|} \cdot
$$

Agora, pela definição da forma complexa $\hat{J}$ dada na Eq. 3.1.14, temos que

$$
2 \mu_{|\bar{\Delta}|}\left(\psi_{1}, \hat{J} \psi_{2}\right)=\Omega\left(\psi_{1}, \psi_{2}\right)
$$

Comparando isso com a definição de $\hat{A}$, dada pela Eq. (7.1.7), e com a da forma bilinear 
$\mu_{|\bar{\Delta}|}$ segue que

$$
-i\left\langle\psi_{1}, \hat{A} \psi_{2}\right\rangle_{|\bar{\Delta}|}=\left\langle\psi_{1},|\hat{A}| \hat{J} \psi_{2}\right\rangle_{|\bar{\Delta}|}
$$

Esse última igualdade é satisfeita se

$$
\hat{J}=-i|\hat{A}|^{-1} \hat{A}
$$

Por fim, assim como feito na Seção 3.1, define-se o mapeamento $K: \mathscr{S} \rightarrow \mathscr{H}_{\text {out }}$ como sendo a restrição a $\mathscr{S} \subset \mathscr{E}^{\prime}$ do projetor espectral de $i \hat{J}$ associado a parte negativa do espectro.

Isso posto, desejo obter uma forma para o mapeamento entre o espaço de soluções da equação de campo e $\mathscr{H}_{\text {out }}$ que dependa explicitamente do operador diferencial espacial que comparece na equação de campo. Essa tarefa é simplificada aplicando-se uma transformação conforme ao espaço-tempo. Considere, então, a transformação definida pela Eq. (5.1.1), com o fator conforme $\Psi=\|\varkappa\|$. Isso leva à Eq. 4.2.16) na equação

$$
-\frac{1}{\|\varkappa\|} \tilde{D}^{a} \tilde{D}_{a} \tilde{X}+\frac{1}{\|\varkappa\|} \tilde{V}_{\mathrm{ef}} \tilde{X}=\sigma \frac{\tilde{X}}{\|\varkappa\|},
$$

com o seguinte potencial efetivo $\tilde{V}_{\mathrm{ef}}$ :

$$
\tilde{V}_{\mathrm{ef}}=\|\varkappa\|^{2} m^{2}+\xi \tilde{R}-\frac{\xi-\frac{1}{6}}{6}\left(\tilde{D}^{a} \tilde{D}_{a} \ln \|\varkappa\|+\tilde{D}_{a} \ln \|\varkappa\| \tilde{D}^{a} \ln \|\varkappa\|\right) .
$$

Ao substituir a nova forma para o operador $\Delta$, que comparece no lado esquerdo da Eq. 7.1.12, no produto interno Eq. (7.1.5) obtém-se

$$
\left\langle\psi_{1}, \psi_{2}\right\rangle_{|\bar{\Delta}|}=\frac{1}{2} \int_{\Sigma_{t}} d \tilde{\Sigma}\left(\overline{\tilde{p}}_{1} \tilde{p}_{2}+\overline{\tilde{\phi}}_{1} \mid \overline{\tilde{\Delta}}_{\mid} \tilde{\phi}_{2}\right)
$$

com $d \tilde{\Sigma}=\sqrt{\hat{h}} d^{3} x$, o elemento de volume associado à métrica espacial conforme $\tilde{h}_{a b}$,

$$
\tilde{\Delta}:=-\tilde{D}^{a} \tilde{D}_{a} \cdot+\tilde{V}_{\mathrm{ef}}
$$

e $\tilde{\phi}$ corresponde ao campo transformado de acordo com a Eq. (5.1.3). Seguindo a referência (41), vou escrever os integrandos que comparecem na forma simplética definida pela Eq. 3.1.5 e no produto interno Eq. 7.1.14 em termos de produtos de matrizes. Essas 
quantidades, então, adquirem a seguintes formas:

$$
\Omega\left(\bar{\psi}_{1}, \psi_{2}\right)=\int_{\Sigma_{t}} d \tilde{\Sigma}\left[\begin{array}{ll}
\overline{\tilde{\phi}}_{1} & \overline{\tilde{p}}_{1}
\end{array}\right]\left[\begin{array}{cc}
0 & -1 \\
1 & 0
\end{array}\right]\left[\begin{array}{c}
\tilde{\phi}_{2} \\
\tilde{p}_{2}
\end{array}\right]
$$

e

$$
\left\langle\psi_{1}, \psi_{2}\right\rangle_{|\bar{\Delta}|}=\frac{1}{2} \int_{\Sigma_{t}} d \tilde{\Sigma}\left[\begin{array}{ll}
\overline{\tilde{\phi}}_{1} & \overline{\tilde{p}}_{1}
\end{array}\right]\left[\begin{array}{cc}
|\overline{\tilde{\Delta}}| & 0 \\
0 & 1
\end{array}\right]\left[\begin{array}{c}
\tilde{\phi}_{2} \\
\tilde{p}_{2}
\end{array}\right] .
$$

Comparando as Eq. 7.1.16 e Eq. 7.1.17 com a definição do operador Â, Eq. 7.1.7, conclui-se que esse operador pode ser realizado como a seguinte matriz de operadores atuando em $L^{2}\left(\Sigma_{t}, d \tilde{\Sigma}\right) \oplus L^{2}\left(\Sigma_{t}, d \tilde{\Sigma}\right)$ :

$$
\hat{A}=2 i\left[\begin{array}{cc}
0 & -|\overline{\tilde{\Delta}}|^{-1} \\
1 & 0
\end{array}\right],
$$

de onde ainda é possível obter que

$$
|\hat{A}|=2\left[\begin{array}{cc}
|\overline{\tilde{\Delta}}|^{-1 / 2} & 0 \\
0 & |\overline{\tilde{\Delta}}|^{-1 / 2}
\end{array}\right] .
$$

Substituindo Eq. 7.1.18 e Eq. 7.1.19 na Eq. 7.1.11 para a forma complexa $\hat{J}$ vem que

$$
\hat{J}=\left[\begin{array}{cc}
0 & -|\overline{\tilde{\Delta}}|^{-1 / 2} \\
|\overline{\tilde{\Delta}}|^{1 / 2} & 0
\end{array}\right] .
$$

Já sabemos que $i \hat{J}=\hat{P}_{+}-\hat{P}_{-}$, por conta da definição da forma complexa, Eq. 3.1.14, e do Teorema Espectral. Uma vez que $\hat{P}_{+}+\hat{P}_{-}=\hat{1}$, é claro que

$$
\hat{P}_{-}=\frac{\hat{1}-i \hat{J}}{2}
$$

o que, em termos das matrizes usadas acimas, significa que

$$
\hat{P}_{-}=\frac{1}{2}\left[\begin{array}{cc}
1 & i|\overline{\tilde{\Delta}}|^{-1 / 2} \\
-i|\overline{\tilde{\Delta}}|^{1 / 2} & 1
\end{array}\right]
$$

Olhemos agora para a forma do produto interno do espaço de Hilbert de uma partícula. Assim como definido pela Eq. (3.1.21), temos que sua relação com o produto interno $\mu_{|\bar{\Delta}|}$ 
é dada por

$$
\left\langle\hat{P}_{-} \psi_{1}, \hat{P}_{-} \psi_{2}\right\rangle_{\mathscr{S}_{\mu_{|\bar{\Delta}|}}^{\mathbb{C}}}=2 \mu_{|\bar{\Delta}|}\left(\overline{\hat{P}_{-} \psi_{1}}, \hat{P}_{-} \psi_{2}\right)=\left\langle\hat{P}_{-} \psi_{1},|\hat{A}| \hat{P}_{-} \psi_{2}\right\rangle_{|\bar{\Delta}|}
$$

onde, na última igualdade, foi usando a definição dada para $\mu_{\mid \bar{\Delta}}$, Eq. (7.1.8). Denotando por $\langle\cdot, \cdot\rangle_{L^{2} \oplus L^{2}}$ o produto interno em $L^{2}\left(\Sigma_{t}, d \tilde{\Sigma}\right) \oplus L^{2}\left(\Sigma_{t}, d \tilde{\Sigma}\right)$,

$$
\left\langle\psi_{1}, \psi_{2}\right\rangle_{L^{2} \oplus L^{2}}=\int_{\Sigma_{t}} d \tilde{\Sigma}\left[\begin{array}{cc}
\bar{\phi}_{1} & \overline{\tilde{p}}_{1}
\end{array}\right]\left[\begin{array}{c}
\tilde{\phi}_{2} \\
\tilde{p}_{2}
\end{array}\right]
$$

teremos para a Eq. (7.1.23) que

$$
\left\langle\hat{P}_{-} \psi_{1}, \hat{P}_{-} \psi_{2}\right\rangle_{\mathscr{S}_{\mu_{\mid} \overline{\mid} \mid}^{\mathbb{C}}}=\frac{1}{2}\left\langle\hat{P}_{-} \psi_{1}, \hat{D}|\hat{A}| \hat{P}_{-} \psi_{2}\right\rangle_{L^{2} \oplus L^{2}}
$$

onde

$$
\hat{D}=\left[\begin{array}{cc}
|\overline{\tilde{\Delta}}| & 0 \\
0 & 1
\end{array}\right]
$$

É possível simplificar a expressão do lado direito da Eq. 7.1.25 ao reescrevê-lo de sorte que seus dois argumentos tenham a mesma forma. De fato, usando que $\hat{D}|\hat{A}|=$ $\sqrt{\hat{D}|\hat{A}|} \sqrt{\hat{D}|\hat{A}|}$ e que $\sqrt{\hat{D}|\hat{A}|}$ é auto-adjunto chega-se em

$$
\left\langle\hat{P}_{-} \psi_{1}, \hat{P}_{-} \psi_{2}\right\rangle_{\mathscr{S}_{\mu_{|\bar{\Delta}|}}^{\mathbb{C}}}=\frac{1}{2}\left\langle\sqrt{\hat{D}|\hat{A}|} \hat{P}_{-} \psi_{1}, \sqrt{\hat{D}|\hat{A}|} \hat{P}_{-} \psi_{2}\right\rangle_{L^{2} \oplus L^{2}}
$$

O produto $\sqrt{\hat{D}|\hat{A}|} \hat{P}_{-}$, por sua vez, tem a seguinte forma:

$$
\sqrt{\hat{D}|\hat{A}|} \hat{P}_{-}=\frac{1}{\sqrt{2}}\left[\begin{array}{cc}
|\overline{\tilde{\Delta}}|^{1 / 4} & i|\overline{\tilde{\Delta}}|^{-1 / 4} \\
-i|\overline{\tilde{\Delta}}|^{1 / 4} & |\overline{\tilde{\Delta}}|^{-1 / 4}
\end{array}\right]
$$

O fruto de todas essas manipulações é a forma abaixo para o produto interno do espaço de Hilbert de uma partícula de interesse:

$$
\left\langle\hat{P}_{-} \psi_{1}, \hat{P}_{-} \psi_{2}\right\rangle_{\mathscr{S}_{\mu_{|\bar{\Delta}|}^{\mathbb{C}}}^{\mathbb{C}}}=\left\langle\frac{|\overline{\tilde{\Delta}}|^{1 / 4} \tilde{\phi}_{1}+i|\overline{\tilde{\Delta}}|^{-1 / 4} \tilde{p}_{1}}{\sqrt{2}}, \frac{|\overline{\tilde{\Delta}}|^{1 / 4} \tilde{\phi}_{2}+i|\overline{\tilde{\Delta}}|^{-1 / 4} \tilde{p}_{2}}{\sqrt{2}}\right\rangle_{L^{2}} .
$$

Isso permite escrever o mapeamento $K: \mathscr{S} \rightarrow \mathscr{H}_{\text {out }}=L^{2}\left(\Sigma_{t}, d \tilde{\Sigma}\right)$ como

$$
K \psi=\frac{|\overline{\tilde{\Delta}}|^{1 / 4} \tilde{\phi}+i|\overline{\tilde{\Delta}}|^{-1 / 4} \tilde{p}}{\sqrt{2}}
$$

onde $\psi=(\phi, \pi), \phi=\frac{\tilde{\phi}}{\|\varkappa\|}$ e $\pi=\|\varkappa\| \sqrt{\tilde{h}} \tilde{p}$. De posse da forma desejada para o mapeamento 
K, Eq. 7.1.30), é possível implementá-lo através dos projetores espectrais do operador $\overline{\tilde{\Delta}}$. Para tanto, é necessário usar a forma dada na Eq. 5.1 .22 para a parte temporal dos modos exponenciais com $\alpha_{n}=\pi / 4$, para todo $n \in \mathcal{O}^{\prime}$. Assim, com os modos $v_{l}$ e $w_{n}^{(\pi / 4)}$ posso escrever uma solução da equação de KG como

$$
\begin{aligned}
\tilde{\phi}(t, \mathbf{x})= & \int_{\mathcal{O}} \frac{d \nu(l)}{\sqrt{2 \varpi_{l}}}\left[\tilde{\phi}_{+}(l) e^{-i \varpi_{l} t}+\tilde{\phi}_{-}(l) e^{i \varpi_{l} t}\right] \tilde{G}_{l}(\mathbf{x}) \\
& +\sum_{n \in \mathcal{O}^{\prime}}\left[c_{n} \frac{e^{\Omega_{n} t-i \pi / 4}+e^{-\Omega_{n} t+i \pi / 4}}{\sqrt{4 \Omega_{n}}}+\bar{c}_{n} \frac{e^{\Omega_{n} t+i \pi / 4}+e^{-\Omega_{n} t-i \pi / 4}}{\sqrt{4 \Omega_{n}}}\right] \tilde{H}_{n}(\mathbf{x})
\end{aligned}
$$

e

$$
\begin{aligned}
& \tilde{p}(t, \mathbf{x})=-i \int_{\mathcal{O}} \frac{d \nu(l)}{\sqrt{2 \varpi_{l}}} \varpi_{l}\left[\tilde{\phi}_{+}(l) e^{-i \varpi_{l} t}-\tilde{\phi}_{-}(l) e^{i \varpi_{l} t}\right] \tilde{G}_{l}(\mathbf{x}) \\
& +\sum_{n \in \mathcal{O}^{\prime}} \Omega_{n}\left[c_{n} \frac{e^{\Omega_{n} t-i \pi / 4}-e^{-\Omega_{n} t+i \pi / 4}}{\sqrt{4 \Omega_{n}}}+\bar{c}_{n} \frac{e^{\Omega_{n} t+i \pi / 4}-e^{-\Omega_{n} t-i \pi / 4}}{\sqrt{4 \Omega_{n}}}\right] \tilde{H}_{n}(\mathbf{x}),
\end{aligned}
$$

onde $\|\varkappa\| G_{l}=\tilde{G}_{l}$ e $\|\varkappa\| H_{n}=\tilde{H}_{n}$. Quando substituídas na Eq. 7.1.30, as formas acima para $\tilde{\phi}$ e $\tilde{p}$ levam a

$$
\left.K \psi\right|_{t=0}=\int_{\mathcal{O}} d \nu(l) \tilde{\phi}_{+}(l) \tilde{G}_{l}(\mathbf{x})+\sum_{n \in \mathcal{O}^{\prime}} c_{n} \tilde{H}_{n}(\mathbf{x}) .
$$

Como já seria de se esperar, tendo em vista o que foi mencionado acima sobre o produto interno Eq. 7.1.5), o resultado desse mapeamento depende da escolha de uma superfície de Cauchy e, portanto, $\mathscr{H}_{\text {out }}$. Para a forma da Eq. 5.1 .22 dos modos exponenciais é adequado escolher a hiperfície $\Sigma_{t=0}$ a fim de que seja possível "separar" o coeficiente $c_{n}$ do coeficiente $\bar{c}_{n}$. Dito de outro modo, a escolha de uma outra hiperfície $\Sigma_{t \neq 0}$ faz com que o resultado da aplicação do operador $\frac{|\overline{\tilde{\Delta}}|^{1 / 4} \cdot+i|\overline{\tilde{\Delta}}|^{-1 / 4}}{\sqrt{2}}$ tenha como imagem um subespaço de $\mathscr{H}_{\text {out }} \oplus \overline{\mathscr{H}}_{\text {out }}$, com $\mathscr{H}_{\text {out }}$ o subespaço de $\mathscr{S}_{\mu_{|\bar{\Delta}|}^{\mathbb{C}}}$ definido pelo mapeamento Eq. 7.1.30. 


\subsection{Da relação entre o efeito e barreiras de potencial parabólicas}

O objetivo desta seção é o estudo dos possíveis valores da energia associada aos graus de liberdade do campo descritos pelos modos $w_{n}$. A expressão formal Eq. 5.4.3 para o operador hamiltoniano em termos dos operadores de criação e destruição da base out mostra que os autovetores de operador número não são, em geral, autovetores de $\hat{H}$. A parte não-diagonal deve-se exclusivamente à existência dos modos exponenciais. Assim, aqui o que interessa são os operadores

$$
\hat{H}_{\Omega_{n}}=-\frac{\sqrt{3}}{2} \Omega_{n}\left(\hat{c}_{n}^{\dagger} \hat{c}_{n}+\hat{c}_{n} \hat{c}_{n}^{\dagger}\right)-\Omega_{n}\left(\hat{c}_{n} \hat{c}_{n}+\hat{c}_{n}^{\dagger} \hat{c}_{n}^{\dagger}\right)
$$

Uma rota possível para se estudar o espectro desses operadores é fazer o caminho inverso daquele adotado nos cursos básicos de Mecânica Quântica. Por simplicidade, considere um dos graus de liberdade do campo correspondente a um modo exponencial. Para esse grau de liberdade escolhido, podemos definir novos operadores $\hat{q}_{\Omega_{n}}$ e $\hat{p}_{\Omega_{n}}$ em analogia aos operadores posição e momentum do oscilador harmônico quântico:

$$
\hat{q}_{\Omega_{n}}:=\frac{\hat{c}_{n}^{\dagger}+\hat{c}_{n}}{\sqrt{2 \Omega_{n}}}
$$

e

$$
\hat{p}_{\Omega_{n}}:=i \sqrt{\frac{\Omega_{n}}{2}}\left(\hat{c}_{n}^{\dagger}-\hat{c}_{n}\right) .
$$

A analogia é construída de sorte que

$$
\left[\hat{q}_{\Omega_{n}}, \hat{p}_{\Omega_{n}}\right]=i \hat{1}
$$

Substituindo as definições Eq. 77.2.2 na Eq. (7.2.1) chega-se na seguinte forma para o hamiltoniano Eq. (7.2.1):

$$
\hat{H}_{\Omega_{n}}=\left(1-\frac{\sqrt{3}}{2}\right) \hat{p}_{\Omega_{n}}^{2}-\left(1+\frac{\sqrt{3}}{2}\right) \Omega_{n}^{2} \hat{q}_{\Omega_{n}}^{2} .
$$


A Eq. 7.2.4 mostra que o operador $\hat{H}_{\Omega_{n}}$ associado a cada grau de liberdade do campo descrito por um modo $w_{n}$ não é nada mais do que o bem-conhecido operador hamiltoniano de uma partícula quântica não-relativística na presença de uma barreira de potencial parabólica (veja, e.g., a referência (51)). Com efeito, é possível realizar o subespaço de $\mathscr{F}_{\mathrm{s}}\left(\mathscr{H}_{\text {out }}\right)$ associado às diferentes ocupações de um modo exponencial em termos do espaço $L^{2}(\mathbb{R}, d q)$. Nessa representação $\hat{H}_{\Omega_{n}}$ é o operador diferencial que define a seguinte equação de Schrödinger independente do tempo:

$$
\left[-\left(1-\frac{\sqrt{3}}{2}\right) \frac{d^{2}}{d q^{2}}-\left(1+\frac{\sqrt{3}}{2}\right) \Omega^{2} q^{2}\right] \psi(q)=E \psi(q) .
$$

Por simplicidade de notação, a partir deste ponto omitirei o índice $n$. Esse operador diferencial é essencialmente auto-adjunto e seu espectro é todo o conjunto dos reais (veja o Teorema 5.10 da referência (52)). Logo, suas autofunções podem ser usadas para expandir qualquer elemento de $L^{2}(\mathbb{R}, d q)$, o conjunto das funções de quadrado integrável com domínio contido nos reais. As soluções da Eq. (7.2.5) são as funções cilíndricas parabólicas. Aqui, entretanto, é mais conveniente expressar as autofunções desse hamiltoniano na seguinte forma:

$$
\begin{aligned}
\psi_{+}(\varepsilon, q)= & \frac{\Omega^{1 / 4}}{\sqrt{2 \pi}} \sqrt{\frac{1+\frac{\sqrt{3}}{2}}{1-\frac{\sqrt{3}}{2}}} \sqrt{\frac{\left|\Gamma\left(\frac{1}{4}-\frac{i \varepsilon}{2}\right)\right|}{\left|\Gamma\left(\frac{3}{4}-\frac{i \varepsilon}{2}\right)\right|}} \frac{1}{\left(1+e^{-2 \pi \varepsilon}\right)^{1 / 4}} \\
& \times e^{-i \sqrt{\frac{1+\sqrt{3} / 2}{1-\sqrt{3} / 2} \frac{\Omega q^{2}}{2}}}{ }_{1} F_{1}\left(\frac{1}{4}+\frac{i \varepsilon}{2} ; \frac{1}{2} ; i \sqrt{\frac{1+\frac{\sqrt{3}}{2}}{1-\frac{\sqrt{3}}{2}} \Omega q^{2}}\right),
\end{aligned}
$$

para as autofunções pares e

$$
\begin{aligned}
\psi_{-}(\varepsilon, q)= & \sqrt{\frac{2}{\pi}} \Omega^{3 / 4}\left(\frac{1+\frac{\sqrt{3}}{2}}{1-\frac{\sqrt{3}}{2}}\right)^{3 / 8} \sqrt{\frac{\left|\Gamma\left(\frac{3}{4}-\frac{i \varepsilon}{2}\right)\right|}{\left|\Gamma\left(\frac{1}{4}-\frac{i \varepsilon}{2}\right)\right|} \frac{e^{i \pi / 4}}{\left(1+e^{-2 \pi \varepsilon}\right)^{1 / 4}}} \\
& \times q e^{-i \sqrt{\frac{1+\sqrt{3} / 2}{1-\sqrt{3} / 2} \frac{\Omega q^{2}}{2}}}{ }_{1} F_{1}\left(\frac{3}{4}+\frac{i \varepsilon}{2} ; \frac{3}{2} ; i \sqrt{\frac{1+\frac{\sqrt{3}}{2}}{1-\frac{\sqrt{3}}{2}} \Omega q^{2}}\right)
\end{aligned}
$$

para as autofunções ímpares. Na Eq. (7.2.6) $\varepsilon:=E / \Omega$ ao passo que $\Gamma(z)$ e ${ }_{1} F_{1}(a ; b ; z)$ denotam, respectivamente, a função gama e a função hipergeométrica confluente ou função de Kummer, como definidas na referência 48 .

Uma vez que temos em mãos as autofunções do hamiltoniano associado ao grau de 


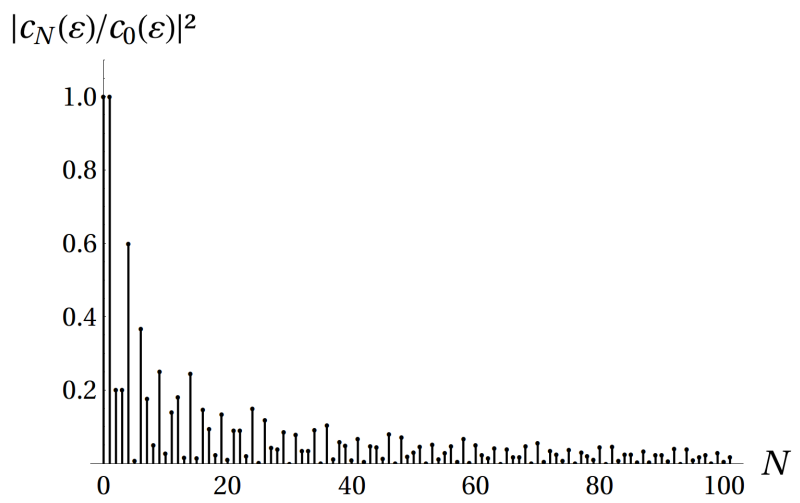

(a)

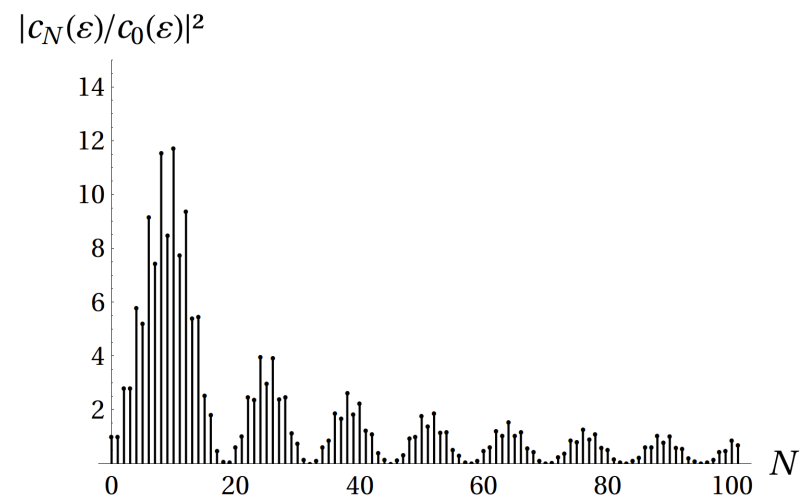

(b)

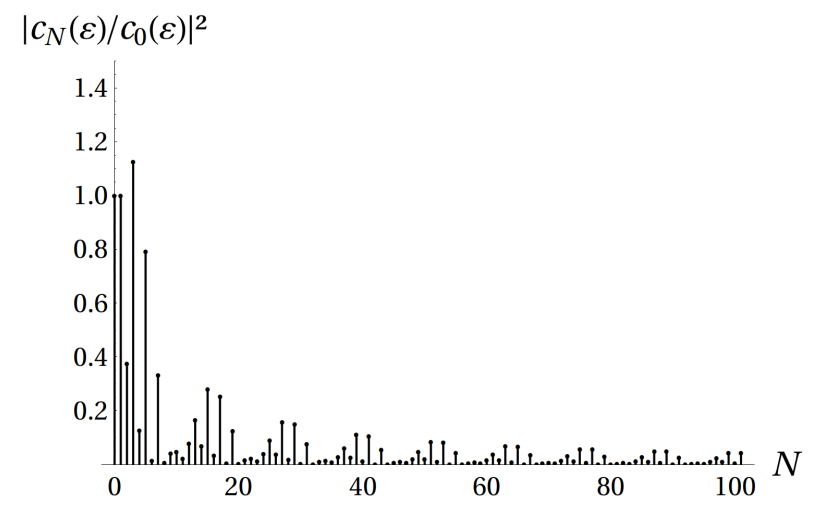

(c)

Figura 7.1 - Distribuição do número de ocupação $N$ para um dado valor de $\varepsilon$. Em cada um dos gráficos o eixo vertical encontra-se normalizado por $\left|c_{0}(\varepsilon)\right|^{2}$ apenas por conveniência. O gráfico em (a) corresponde a $\varepsilon=-1.5$, o em (b) a $\varepsilon=1.5$ e o em (c) a $\varepsilon=0$.

liberdade do campo quântico representado por $w_{n}$, podemos nos perguntar qual a distribuição de energia num estado com número bem-definido de quanta desse modo. Como é bem-sabido, os vetores do espaço de Fock com um certo número de ocupação de um grau de liberdade do campo podem ser realizados em termos dos autovetores do hamiltoniano do oscilador harmônico quântico. Assim sendo, o estado de ocupação $N$ de um modo exponencial, o vetor $\left|N_{\Omega}\right\rangle$, é representado em $L^{2}(\mathbb{R}, d q)$ como

$$
\varphi_{N}(q):=\left\langle q \mid N_{\Omega}\right\rangle=\left(\frac{\Omega}{\pi}\right)^{1 / 4} \frac{1}{\sqrt{2^{N} \pi N !}} e^{-\frac{\Omega q^{2}}{2}} H_{N}(\sqrt{\Omega} q),
$$

onde $H_{N}(z)$ denota o polinômio de Hermite de grau $N$, como definido na referência (48). Da mesma forma que antes, é também conveniente escrever os polinômios de Hermite em termos das funções de Kummer. Para os de grau par

$$
\varphi_{2 m}(q)=\left(\frac{\Omega}{\pi}\right)^{1 / 4} \frac{(-1)^{m}}{m !} \sqrt{\frac{(2 m) !}{4^{m}}} e^{-\frac{\Omega q^{2}}{2}}{ }_{1} F_{1}\left(-m ; \frac{1}{2} ; \Omega q^{2}\right),
$$




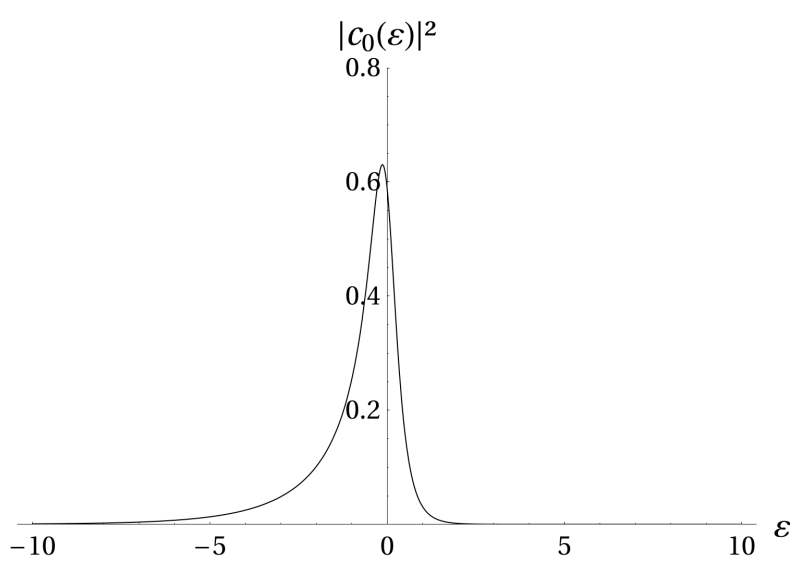

(a)

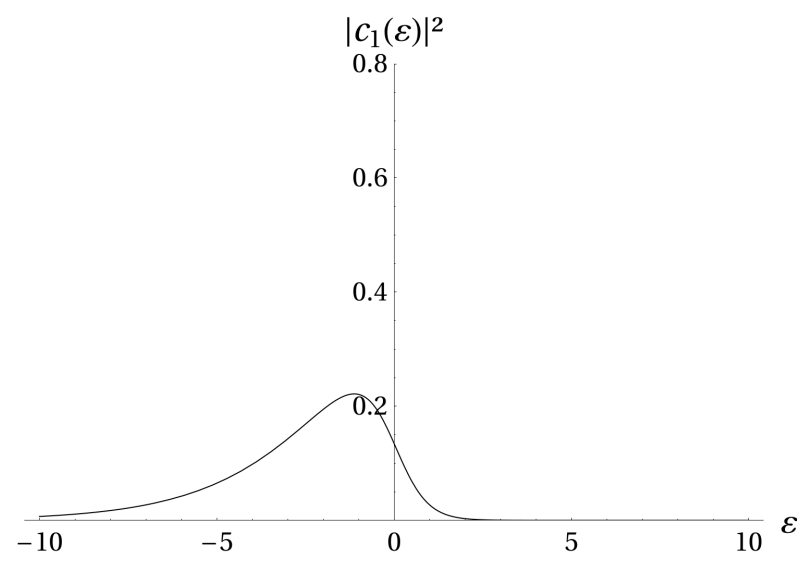

(b)

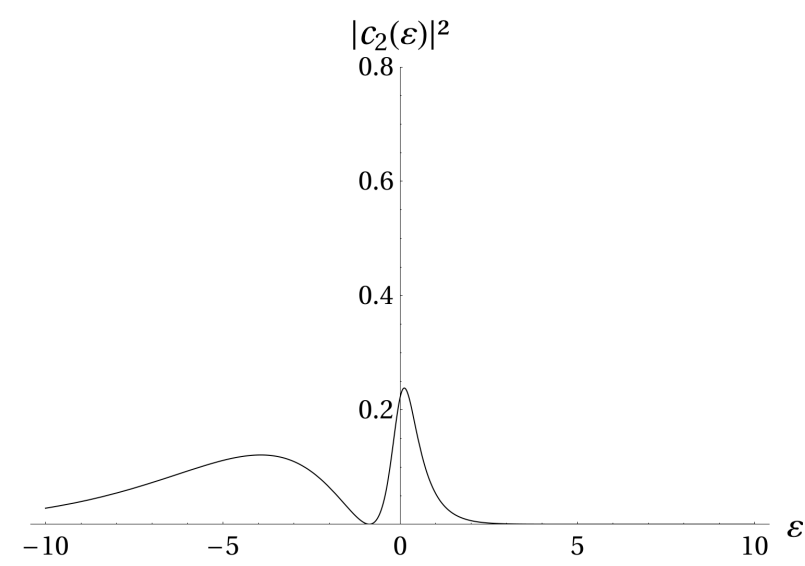

(c)

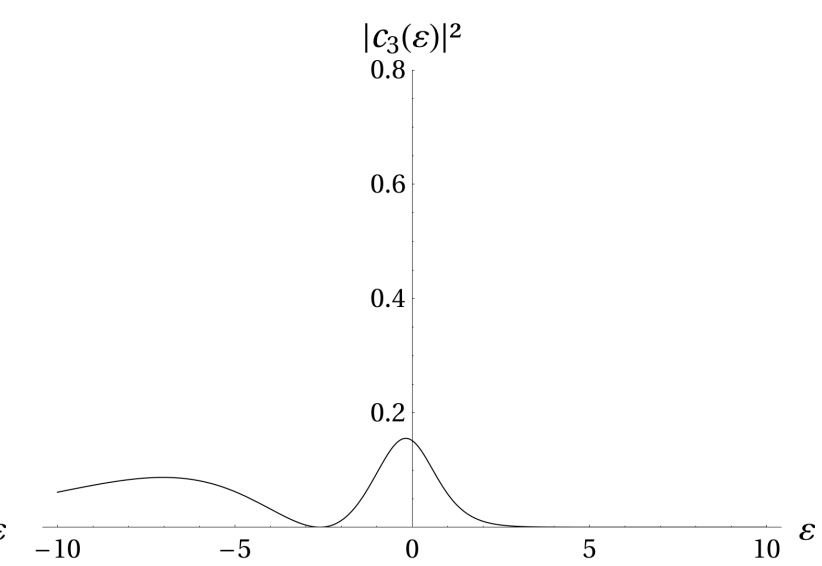

(d)

Figura 7.2 - Distribuição de energia em um estado com um dado número de quanta do modo $w_{\Omega}$. O gráfico em (a) corresponde a $N=0$, o estado de vácuo, o em (b) a $N=1$, o em (c) a $N=2$ e o em (d) a $N=3$.

enquanto que para os de grau ímpar

$$
\varphi_{2 m+1}(q)=\left(\frac{4 \Omega^{3}}{\pi}\right)^{1 / 4} \frac{(-1)^{m}}{m !} \sqrt{\frac{(2 m+1) !}{4^{m}}} q e^{-\frac{\Omega q^{2}}{2}}{ }_{1} F_{1}\left(-m ; \frac{3}{2} ; \Omega q^{2}\right),
$$

com $m \in \mathbb{N}$. Dada a representação do vetor $\left|N_{\Omega}\right\rangle$ em $L^{2}(\mathbb{R}, d q)$, pode-se calcular sua projeção nas autofunções de $\hat{H}_{\Omega}$. De posse da Eq. (7.2.6 para $\psi_{ \pm}$e da Eq. (7.2.8) para $\varphi_{N}$, as projeções $c_{2 m}(\varepsilon):=\left\langle(2 m)_{\Omega} \mid \psi_{+}(\varepsilon)\right\rangle$ e $c_{2 m+1}(\varepsilon):=\left\langle(2 m+1)_{\Omega} \mid \psi_{-}(\varepsilon)\right\rangle$ - calculadas 
com o auxílio da fórmula 7.622.1 da referência $(53)$ — têm a seguinte forma:

$$
\begin{aligned}
c_{2 m}(\varepsilon)= & \frac{1}{\pi^{3 / 4}} \frac{1}{m !} \sqrt{\frac{(2 m) !}{4^{m}}}\left(\frac{1+\frac{\sqrt{3}}{2}}{1-\frac{\sqrt{3}}{2}}\right)^{1 / 8} \frac{\Gamma\left(\frac{1}{2}\right)}{\left(1+e^{-2 \pi \varepsilon}\right)^{1 / 4}} \sqrt{\frac{\left|\Gamma\left(\frac{1}{4}-\frac{i \varepsilon}{2}\right)\right|}{\left|\Gamma\left(\frac{3}{4}-\frac{i \varepsilon}{2}\right)\right|}} \\
& \times\left[\frac{1-i \sqrt{\frac{1+\sqrt{3} / 2}{1-\sqrt{3} / 2}}}{1+i \sqrt{\frac{1+\sqrt{3} / 2}{1-\sqrt{3} / 2}}}\right]\left|\left[1+i \sqrt{\frac{1+\sqrt{3} / 2}{1-\sqrt{3} / 2}}\right]^{-1 / 4+i \varepsilon / 2}\right|^{2} \mid\left[-\frac{1}{4}+\frac{i \varepsilon}{2} ; \frac{1}{2} ; \frac{-4 i \sqrt{\frac{1+\sqrt{3} / 2}{1-\sqrt{3} / 2}}}{\left[1-i \sqrt{\frac{1+\sqrt{3} / 2}{1-\sqrt{3} / 2}}\right]^{2}}\right) \\
& \times F\left(-m, \frac{1}{[1}\right)
\end{aligned}
$$

$\mathrm{e}$

$$
\begin{aligned}
c_{2 m+1}(\varepsilon)= & \frac{2^{5 / 2}}{\pi^{3 / 4}} \frac{1}{m !} \sqrt{\frac{(2 m+1) !}{4^{m}}}\left(\frac{1+\frac{\sqrt{3}}{2}}{1-\frac{\sqrt{3}}{2}}\right)^{3 / 8} \frac{\Gamma\left(\frac{3}{2}\right) e^{i \pi / 4}}{\left(1+e^{-2 \pi \varepsilon}\right)^{1 / 4}} \sqrt{\frac{\left|\Gamma\left(\frac{3}{4}-\frac{i \varepsilon}{2}\right)\right|}{\left|\Gamma\left(\frac{1}{4}-\frac{i \varepsilon}{2}\right)\right|}} \\
& \times\left[\frac{1-i \sqrt{\frac{1+\sqrt{3} / 2}{1-\sqrt{3} / 2}}}{1+i \sqrt{\frac{1+\sqrt{3} / 2}{1-\sqrt{3} / 2}}}\right]^{m}\left|\left[1+i \sqrt{\frac{1+\sqrt{3} / 2}{1-\sqrt{3} / 2}}\right]^{-3 / 4+i \varepsilon / 2}\right|^{2} \\
& \times F\left(-m, \frac{3}{4}+\frac{i \varepsilon}{2} ; \frac{3}{2} ; \frac{-4 i \sqrt{\frac{1+\sqrt{3} / 2}{1-\sqrt{3} / 2}}}{\left[1-i \sqrt{\frac{1+\sqrt{3} / 2}{1-\sqrt{3} / 2}}\right]^{2}}\right)
\end{aligned}
$$

onde $F(a, b ; c ; z)$ denota a função hipergeométrica, como definida na referência $(48)$. Na Fig. 7.1 mostro a distribuição dos números de ocupação $N$ para $\varepsilon=-1.5$ em 7.1(a), $\varepsilon=1.5 \mathrm{em} \mathrm{7.1( \textrm {b } )}$ e $\varepsilon=0 \mathrm{em} 7.1(\mathrm{c})$. Já na Fig. 7.2 é apresentada a distribuição de energia para estados do campo com ocupação $N=0$ (vácuo) em $7.2(\mathrm{a}), N=1 \mathrm{em}$ 7.2(b) $N=2 \mathrm{em} 7.2(\mathrm{c})$ e $N=3 \mathrm{em} 7.2(\mathrm{~d})$, A Fig. 7.2, em particular, mostra um fato bastante curioso. Como já foi mencionado acima, o espectro do operador $\hat{H}_{\Omega}$ é todo o conjunto dos reais, o que significa que o grau de liberdade do campo quântico descrito por um modo exponencial pode ter energia arbitrariamente negativa. Isso é conseqüência do perfil do potencial que comparece na Eq. (7.2.5): uma parábola de concavidade negativa. Daí vem o resultado mostrado na Fig. 7.2; durante a fase em que o campo quântico é instável, os graus de liberdade do campo descritos pelos modos $w_{n}$ num estado com número de ocupação bem-definido podem ter energia arbitrariamente negativa. Note, por fim, que o gráfico apresentado na Fig. 7.2(a) apresenta um pico para $\varepsilon<0$. Esse pico é 
consistente com a contribuição dos modos $w_{n}$ ao valor esperado no vácuo da energia do campo assintoticamente no futuro calculada na Seção 5.4 .

A análise apresentada nesta seção tem como subproduto uma outra representação das relações de Weyl Eq. 3.1.8 para o campo escalar quando o vácuo é despertado. Em primeiro lugar vou separar o espaço $\mathscr{S}_{\mu_{|\bar{\Delta}|}}$ em uma parte que contenha somente soluções

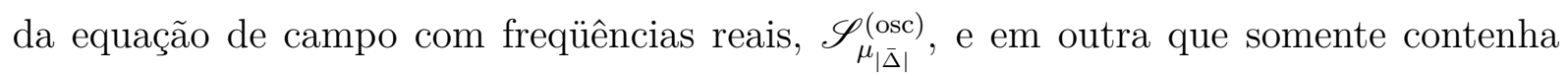

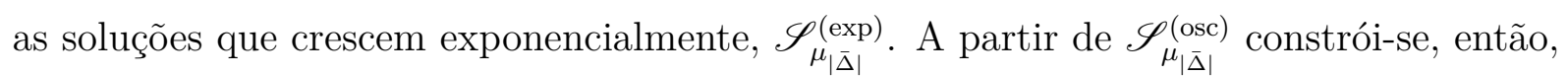
o espaço de Hilbert de uma partícula $\mathscr{H}^{(\text {osc })}$ e dele o espaço de Fock $\mathscr{F}_{\mathrm{s}}\left(\mathscr{H}^{(\text {osc })}\right)$, como mostrado no Capítulo 3. Para os graus de liberdade do campo representados pelos modos $w_{n}$ associa-se o espaço de estado $L^{2}\left(\mathbb{R}, d q_{n}\right)$, como usualmente feito em Mecânica Quântica. Assim, nessa representação o espaço de estados do campo pode ser escrito como

$$
\mathscr{F}_{\mathrm{s}}\left(\mathscr{H}^{(\mathrm{osc})}\right) \otimes\left(\bigotimes_{n \in \mathcal{O}^{\prime}} L^{2}\left(\mathbb{R}, d q_{n}\right)\right) .
$$

Essa representação deixa claro que durante o período de crescimento exponencial das flutuações quânticas não é possível definir um estado de vácuo para o campo. Dito de outro modo, a instabilidade faz com que a energia do sistema não seja limitada inferiormente. 


\section{Capítulo 8}

\section{Da criação de partículas}

$\mathrm{H}$

Á dois pontos que devem ser examinados no que tange o entendimento da relação entre o Efeito de Despertar do Vácuo e fenômenos de criação de partículas. O primeiro deles é a questão da possibilidade de se associar ao sistema algum conteúdo de partícula enquanto o campo passa pelo período durante o qual suas flutuações crescem sem-limites. O segundo ponto tem a ver com a conciliação de dois fatos e, em última instância, com o processo de criação de partículas em si. Por um lado, é sabido que evoluções suficientemente lentas de espaços-tempos bem-comportados não levam à produção de partículas (5,8). Por outro, o desencadeamento do Efeito de Despertar do Vácuo independe do quão lentamente o sistema gravitacional evoluiu até a configuração capaz o fazer.

Para iniciar a discussão farei, na Seção 8.1, uma breve revisão da literatura no que diz respeito à criação de partículas em espaços-tempos curvos, aos moldes da discussão conduzida na referência (5). Em seguida, a fim de abordar a primeira questão, utilizarei, na seção 8.2, um detector de partículas acoplado com o campo. Esse tipo de abordagem segue uma postura muito bem expressa pelo seguinte aforismo (54): "Partículas são aquilo que detectores de partículas detectam." Por fim, para lidar com a segunda questão posta acima investigarei, na seção 8.3 , a contribuição do efeito ao conteúdo de partículas numa fase posterior a presença das instabilidades. Esse tipo de construção é motivada pela 
expectativa de que em algum momento a retroação do campo quântico sobre a matéria clássica deve levar todo o sistema a atingir um novo estado estacionário.

\subsection{Criação de partículas em espaços-tempos curvos}

Antes de expor o mecanismo de criação de partículas em TQCEC, acredito ainda caber mais alguns comentários acerca do conceito de partícula nesse contexto. O ponto fundamental que deve ser destacado sobre o conceito (global) de partícula, como descritas pela Teoria Quântica de Campos, é a relação de dependência entre ele e eventuais simetrias globais do espaço-tempo de fundo. Essa relação de dependência foi tangenciada ao final da Seção 3.2. Lá foi mencionado que a prescrição de quantização ali delineada deságua em um espaço de estados para o campo que possui naturalmente a interpretação de descrever estados de ocupação de partículas. Nessa linha, o caso arquetípico é o de quando o espaço-tempo de fundo é o de Minkowski. Uma vez que a finalidade é tornar a Mecânica Quântica consistente com os princípios da Relatividade Restrita, é necessário fazer com que suas leis sejam invariantes sob o grupo de Poincaré. Essa imposição leva à definição de que os estados de uma partícula devem ser vetores de um espaço sobre o qual se concretizam representações irredutíveis daquele grupo de simetrias (11). Ademais, isometrias diferentes do mesmo espaço-tempo podem levar a conceitos distintos de partícula. Esse é o caso, por exemplo, do Efeito Unruh (55), onde, mesmo quando observadores inerciais não detectam nenhuma partícula ao realizarem medidas sobre o estado de vácuo de um campo quântico, observadores uniformemente acelerados detectam um banho térmico de partículas desse campo a uma temperatura proporcional à sua aceleração própria.1 $\left.\right|^{1}$

Isso posto, considere um espaço-tempo de fundo que é assintoticamente estacionário tanto no passado quanto no futuro. Por simplicidade, suponha que o estado inicial do campo quântico é o estado de vácuo $\Psi_{0}^{\text {in }}$. Do ponto de vista dos observadores que no passado seguem a isometria temporal daquela região o operador $\hat{a}_{k}^{\dagger} \hat{a}_{k}$ conta o número de

\footnotetext{
${ }^{1}$ Para uma revisão sobre o Efeito Unruh veja, e.g., a referência (56).
} 
quanta de energia do campo que ocupam o estado descrito por $\varphi_{k}^{\text {in }}$. Pela definição dada na Eq. (3.1.33) para o estado de vácuo tem-se que no passado

$$
\left\langle 0_{\text {in }}\left|\hat{a}_{k}^{\dagger} \hat{a}_{k}\right| 0_{\text {in }}\right\rangle:=\left\langle\Psi_{0}^{\text {in }}, \hat{a}_{k}^{\dagger} \hat{a}_{k} \Psi_{0}^{\text {in }}\right\rangle_{\mathscr{F}_{\mathrm{s}}\left(\mathscr{H}_{\text {in }}\right)}=0
$$

isto é, do ponto de vista desses observadores, o estado $\Psi_{0}^{\text {in }}$ no passado corresponde à ausência de quanta do campo. Já no futuro é o operador $\hat{b}_{l}^{\dagger} \hat{b}_{l}$ que, no entender dos observadores que seguem a isometria temporal naquela região, cumpre a tarefa de contar quanta de energia do campo ocupando o estado $\varphi_{l}^{\text {out }}$. Desse modo, se $\Psi_{0}^{\text {in }}$ é o estado inicial do campo, o valor esperado para número de partículas criadas nesse estado pela mudança da geometria de fundo é dado por

$$
\begin{aligned}
\left\langle 0_{\text {in }}\left|\hat{b}_{l}^{\dagger} \hat{b}_{l}\right| 0_{\text {in }}\right\rangle & :=\left\langle\Psi_{0}^{\text {in }},\left(\sum_{k \in I} \bar{A}_{k l} \hat{a}_{k}^{\dagger}-\bar{B}_{k l} \hat{a}_{k}\right)\left(\sum_{k^{\prime} \in I} A_{k^{\prime} l} \hat{a}_{k^{\prime}}-B_{k^{\prime} l} \hat{a}_{k^{\prime}}^{\dagger}\right) \Psi_{0}^{\text {in }}\right\rangle_{\mathscr{F}_{\mathrm{s}}\left(\mathscr{G}_{\text {in }}\right)} \\
& =\sum_{k \in I}\left|B_{k l}\right|^{2}
\end{aligned}
$$

onde foi usada a transformação de Bogoliubov como definida na Eq. (3.3.5) e o coeficiente de Bogoliubov $B_{k l}$ é definido pela Eq. (3.3.2b). No caso estático é ainda possível escrever as bases de $\mathscr{H}_{\text {in }}$ e $\mathscr{H}_{\text {out }}$, respectivamente, em termos dos modos de freqüência positiva no passado e no futuro, assim como mostrado na Seção 3.3. Isso leva a

$$
\left\langle 0_{\text {in }}\left|\hat{b}_{l}^{\dagger} \hat{b}_{l}\right| 0_{\text {in }}\right\rangle=\sum_{k \in I}\left|B_{k l}\right|^{2}=\int_{\mathcal{I}} d \mu(j) \int_{\mathcal{O}} d \nu(p) d \nu\left(p^{\prime}\right) \tilde{\varphi}_{l}^{\text {out }}(p) \tilde{\varphi}_{l}^{\text {out }}\left(p^{\prime}\right) \beta_{p j} \bar{\beta}_{p^{\prime} j}
$$

ao se usar a relação entre o coeficiente $B_{k l}$ e o coeficiente $\beta_{p j}$ Eq. (3.3.11b) e que

$$
\sum_{k \in I} \tilde{\varphi}_{k}^{\text {in }}(j) \tilde{\varphi}_{k}^{\text {in }}\left(j^{\prime}\right)=\delta_{\mu}\left(j, j^{\prime}\right)
$$

esta última conseqüência da completeza da base in. O número total de partículas produzidas pela evolução do espaço-tempo de fundo então fica

$$
\sum_{l \in O}\left\langle 0_{\text {in }}\left|\hat{b}_{l}^{\dagger} \hat{b}_{l}\right| 0_{\text {in }}\right\rangle=\int_{\mathcal{I}} d \mu(j) \int_{\mathcal{O}} d \nu(p)\left|\beta_{p j}\right|^{2}
$$

Pelo resultado da Eq. 8.1.2 nota-se que a produção de partículas se dá se, e somente se, a evolução do espaço-tempo de fundo é capaz de fazer com que os vetores de $\mathscr{H}_{\text {in }}$ só possam ser expressos como combinação de vetores de $\mathscr{H}_{\text {out }}$ e $\overline{\mathscr{H}}_{\text {out }}$. No caso estático, 
essa condição implica que a produção de partícula depende de a evolução do espaçotempo ser capaz de fazer com que modos de freqüência positiva no passado serem uma mistura de modos de freqüência positiva e negativa no futuro. Grosseiramente falando, essa "transmutação" de um modo de freqüência positiva no passado ocorre quando a escala de tempo de mudança da geometria de fundo é da ordem do inverso de sua freqüência $(5,8)$. Esse mecanismo está na raiz tanto de fenômenos de criação de partículas em universos em expansão (19,57) quanto do efeito descoberto por Hawking (20,58).

\subsection{Excitação de detectores}

Considere um detector que consiste de um sistema quântico com dois níveis de energia, conhecido na literatura por detector de Unruh-DeWitt (5,59), que é imaginado interagir com o campo quântico. As dimensões do detector serão tomadas como muito menores que a escala de distância típica do gradiente do campo gravitacional. Para o campo quântico vou supor novamente o cenário desenhado no Capítulo 5, a saber, um espaço-tempo de fundo que é conformalmente estático e que tanto no passado quando no futuro é tal que o potencial efetivo que comparece na Eq. (5.1.4) não depende, tanto no passado quanto no futuro, da coordenada temporal. Suponha ainda que o aparato é ligado no futuro, a partir do momento em que $V_{\text {ef }}$ não depende de $t$, e que assim permanece por um intervalo de tempo próprio T. O hamiltoniano do sistema total é dado por

$$
\hat{H}_{\text {total }}=\hat{H}+\hat{H}_{\mathrm{D}}+\hat{H}_{\text {int }}
$$

onde $\hat{H}$ é o hamiltoniano do campo livre, $\hat{H}_{\mathrm{D}}$ é o hamiltoniano responsável pela evolução dos graus de liberdade internos do detector e $\hat{H}_{\text {int }}$ modela a interação entre o campo e o detector. Para um detector de Unruh-DeWitt, $\hat{H}_{\mathrm{D}}$ pode ser colocado na forma

$$
\hat{H}_{\mathrm{D}}=\left(E_{1}-E_{0}\right) \hat{C}^{\dagger} \hat{C}+E_{0} \hat{1}
$$


sendo que o operador $\hat{C}$ atua nos auto-estados de energia do detector de maneira que

$$
\hat{C}\left|E_{0}\right\rangle=0 \quad \text { e } \hat{C}\left|E_{1}\right\rangle=\left|E_{0}\right\rangle
$$

O vetor $\left|E_{0}\right\rangle$ denota o estado fundamental do detector e $\left|E_{1}\right\rangle$ seu estado excitado. Já o acoplamento entre o detector e o campo é definido por

$$
\hat{H}_{\mathrm{int}}=\varepsilon(\tau) \int_{\Sigma_{t}} \hat{\phi}(\mathbf{x})[F(\mathbf{x}) \hat{C}+\text { h.c. }] d \Sigma
$$

onde $\varepsilon \in C_{0}^{\infty}(\mathbb{R})$ é uma função responsável por "ligar e desligar" o detector, $F \in C_{0}^{\infty}(\Sigma)$ pode ser imaginado como descrevendo o perfil espacial da ponta de prova do aparato e $\tau$ denota o tempo próprio do detector.

Fixado o sistema físico total, desejo calcular — em ordem mais baixa em $\varepsilon$ - a taxa de excitação do detector quando o vácuo do campo é despertado. Para tanto, é conveniente trabalhar na chamada representação de interação. Como é bem-sabido, nessa representação os operadores evoluem segundo o hamiltoniano livre enquanto que o estado do sistema evolui segundo o hamiltoniano de interação. Assim, o hamiltoniano de interação tem a forma

$$
\hat{H}_{\mathrm{int}}^{(\mathrm{I})}=\varepsilon(\tau) \int_{\Sigma_{t}} \hat{\phi}^{(\mathrm{I})}(t, \mathbf{x})\left[F(\mathbf{x}) e^{-i\left(E_{1}-E_{0}\right) \tau} \hat{C}^{(\mathrm{S})}+\text { h.c. }\right] d \Sigma
$$

na representação de interação e a evolução dos estados é dada, em ordem mais baixa em $\varepsilon$, por

$$
|\mathrm{f}\rangle=\left(\hat{1}-i \int_{0}^{T} \hat{H}_{\text {int }}^{(\mathrm{I})} d \tau\right)|\mathrm{i}\rangle .
$$

Aqui, o sobrescrito (I) indica o operador na representação de interação enquanto que o sobrescrito (S) refere-se a representação de Schrödinger. Para o hamiltoniano de interação dado na Eq. 8.2.5 é possível reorganizar o integrando que comparece na Eq. 8.2.6 de sorte que

$$
\int_{0}^{T} \hat{H}_{\mathrm{int}}^{(\mathrm{I})} d \tau=\hat{\Phi}(f) \hat{C}^{(\mathrm{S})}+\text { h.c. }
$$

onde $f \in C_{0}^{\infty}(\mathcal{M})$ é a seguinte função complexa:

$$
f(t, \mathbf{x}):=\varepsilon[\tau(t)] e^{-i\left(E_{1}-E_{0}\right) \tau(t)} F(\mathbf{x}) .
$$


Uma vez que na representação de interação o campo evolui segundo a hamiltoniana livre, o operador campo, a partir da definição da Eq. 3.1.30, pode ser escrito como

$$
\hat{\Phi}(f)=i \hat{a}(\overline{K E \bar{f}})-i \hat{a}^{\dagger}(K E f)
$$

Já que a finalidade da introdução do detector é o estudo do comportamento do campo devido à geometria de fundo, aqui não interessa o que ocorre com o sistema detector-campo devido ao liga-e-desliga do primeiro. Para contornar os efeitos colaterais do liga-e-desliga do aparato, suponha que isso é feito lentamente. Isso significa que

$$
\left|\frac{\dot{\varepsilon}}{\varepsilon}\right| \ll E_{1}-E_{0}
$$

Essa imposição faz com que $f$ seja aproximadamente de freqüência positiva, de sorte que

$$
K E \bar{f} \approx 0 \quad \text { e } K E f \approx E f
$$

Assim, a Eq. 8.2.6 reduz-se a

$$
|\mathrm{f}\rangle=\left[\hat{1}+\hat{a}^{\dagger}(\lambda) \hat{C}^{(\mathrm{S})}-\hat{a}(\bar{\lambda}) \hat{C}^{\dagger(\mathrm{S})}\right]|\mathrm{i}\rangle
$$

onde $\lambda:=-K E f$. Para prosseguir é necessário reescrever os operadores $\hat{a}(\bar{\lambda})$ e $\hat{a}^{\dagger}(\lambda)$ com o auxílio da base completa para $\mathscr{H}_{\text {out }}$, como definida no Capítulo 5. Utilizando Eq. 3.1.37 e Eq. 3.1.38 vem que

$$
\begin{aligned}
\hat{a}(\bar{\lambda}) & =i \sum_{l \in O} \int_{\mathcal{M}} \sqrt{-g} d^{4} x \bar{f} \varphi_{l}^{\text {out }} \hat{b}_{l} \\
& =i \sum_{\substack{l \in O \\
k \in I}} \int_{\mathcal{M}} \sqrt{-g} d^{4} x \bar{f} \varphi_{l}^{\text {out }}\left(A_{k l} \hat{a}_{k}-B_{k l} \hat{a}_{k}^{\dagger}\right)
\end{aligned}
$$

e que

$$
\begin{aligned}
\hat{a}^{\dagger}(\lambda) & =-i \sum_{l \in O} \int_{\mathcal{M}} \sqrt{-g} d^{4} x f \bar{\varphi}_{l}^{\text {out }} \hat{b}_{l}^{\dagger} \\
& =-i \sum_{\substack{l \in O \\
k \in I}} \int_{\mathcal{M}} \sqrt{-g} d^{4} x f \bar{\varphi}_{l}^{\text {out }}\left(\bar{A}_{k l} \hat{a}_{k}^{\dagger}-\bar{B}_{k l} \hat{a}_{k}\right)
\end{aligned}
$$

A segunda igualdade na Eq. 8.2.13 é obtida usando-se a transformação de Bogoliubov para os operadores de destruição e criação, como definida na Eq. (3.3.5). 
Suponha que no passado o estado do campo é o vácuo de $\mathscr{F}_{\mathrm{s}}\left(\mathscr{H}_{\mathrm{in}}\right)$, o vetor $\Psi_{0}^{\text {in }}$, e que o detector encontra-se em seu estado fundamental. Substituindo a expressão dos operadores de destruição e criação Eq. (8.2.13) na Eq. (8.2.6), vem que o estado final do sistema será

$$
|\mathrm{f}\rangle=\Psi_{0}^{\mathrm{in}} \otimes\left|E_{0}\right\rangle+i \sum_{\substack{l \in O \\ k \in I}} \int_{\mathcal{M}} \sqrt{-g} d^{4} x \bar{f} \varphi_{l}^{\text {out }} B_{k l} \hat{a}_{k}^{\dagger} \Psi_{0}^{\mathrm{in}} \otimes\left|E_{1}\right\rangle .
$$

Assim, a probabilidade de excitação do detector, em primeira ordem de perturbação, depois que este permaneceu ligado por um tempo $T$ é dada por

$$
P_{\mathrm{exc}}=\sum_{k \in I}\left|\sum_{l \in O} \int_{\mathcal{M}} \sqrt{-g} d^{4} x \bar{f} \varphi_{l}^{\text {out }} B_{k l}\right|^{2} .
$$

Se o aparato permanece ligado por um tempo suficientemente longo, isto é, se para o menor autovalor do operador Eq. 5.3.1), $\bar{\Omega}, T$ é tal que $\bar{\Omega} T / \sqrt{-g_{00}} \gg 1$, então a principal contribuição à probabilidade de excitação do detector estará associada à presença dos modos exponenciais. Nesse regime a Eq. 8.2.15 para a probabilidade de excitação do detector reduz-se a

$$
\begin{aligned}
P_{\mathrm{exc}} & \approx \frac{1}{2 \bar{\Omega}} \sum_{k \in I}\left|\int_{0}^{T / \sqrt{-g_{00}}} \varepsilon[\tau(t)] e^{\left(\bar{\Omega}+i \Delta E \sqrt{-g_{00}}\right) t} d t \times \int_{\Sigma_{t}} d \tilde{\Sigma} \frac{F \bar{H}}{\|\tilde{\mathcal{H}}\|} B_{k \bar{\Omega}}\right|^{2} \\
& \approx \varepsilon(T / 2)^{2} \frac{e^{\frac{2 \bar{\Omega} T}{\sqrt{-g_{00}}}}}{2\left(-g_{00}\right) \bar{\Omega}\left[\left(\frac{\bar{\Omega}}{\sqrt{-g_{00}}}\right)^{2}+\Delta E^{2}\right]}\left(\int_{\Sigma_{t}} d \tilde{\Sigma} \frac{F \bar{H}}{\|\tilde{\mathcal{K}}\|}\right)^{2} \sum_{k \in I}\left|B_{k \bar{\Omega}}\right|^{2} .
\end{aligned}
$$

Aqui $g_{00}$ denota a componente temporal do tensor métrico, $\Delta E:=E_{1}-E_{0}$ e $B_{k \bar{\Omega}}$ é o coeficiente de Bogoliubov definido na Eq. 3.3.2b e dá a projeção do conjugado de cada vetor da base in no modo $w_{\bar{\Omega}}$. O campo de Killing $\tilde{\varkappa}^{a}$ já foi definido no Capítulo 5.

A forma final para a probabilidade de excitação do detector dada na Eq. 8.2.16 revela que assintoticamente a probabilidade de excitação do detector, em primeira ordem de perturbação, é totalmente dominada pelo crescimento exponencial das flutuações do campo. Isso acontece independentemente de qual seja a separação entre os níveis de energia do detector e eclipsando a contribuição para a probabilidade de excitação que vem da produção de quanta dos outros modos por conta da evolução do espaço-tempo.

Em face a graus de liberdade do campo quântico descritos por modos tão pouco usuais 
em teoria de campos quanto os modos $w_{n}$, a questão que se coloca é a seguinte: estaria a probabilidade de excitação dada pela Eq. (8.2.16) revelando um fenômeno de produção de partículas em profusão? Dito de outro modo, é possível usar os cliques do detector para conferir ao sistema um certo número de partículas criadas?

Em Teoria Quântica de Campos é sempre tomado como dado que os vetores do espaço de Fock de estados do campo com número de ocupação bem-definido descrevem aquele número de partículas ocupando um certo estado. No entanto, como já repisado algumas vezes, essa é uma interpretação daquele número e não uma definição vinda da prescrição de quantização de campos, de modo que esse entendimento não deve ser visto como um a priori. Em espaço plano, essa interpretação é motivada por dois fatos distintos. Em primeiro lugar, porque quando o espaço-tempo é estacionário os vetores do espaço de Fock são autovetores do operador hamiltoniano livre associado ao campo, o que significa dizer, entre outras coisas, que o número de quanta naquele estado é conservado pela dinâmica livre. Em segundo lugar, porque detectores de partículas, como o de Unruh-DeWitt, têm sua probabilidade de excitação proporcional àquele número de ocupação. Pela discussão conduzida no Capítulo 7, contudo, é claro que ao menos a primeiro fato falha para o espaço $\mathscr{F}_{\mathrm{s}}\left(\mathscr{H}_{\text {out }}\right)$ justamente por conta do setor associado aos modos exponenciais. Assim sendo, absolutamente nada pode ser dito sobre o conteúdo de partículas do sistema durante a fase em que as flutuações do vácuo crescem sem-limites.

\subsection{Criação de partículas quando o vácuo adormece}

Acredito que as discussões conduzidas no Capítulo 7 e, particularmente, na última seção são suficientes para convencer o leitor de que não é possível associar um conteúdo de partículas ao campo enquanto o vácuo encontra-se acordado. No entanto, não é de se esperar que essa fase de instabilidade se perpetue. Com efeito, acreditamos que se considerada, a retroação do campo deve conduzir todo o sistema para um novo regime estacionário. Atingido esse novo regime, é legítimo nos perguntarmos sobre o conteúdo de partículas 
do sistema. Nesta seção estou interessado na contribuição dada pelo Efeito de Despertar do Vácuo para a produção de partículas. É claro que essa é uma questão que somente pode ser fechada lançando-se mão da retroação do campo quântico sobre o espaço-tempo de fundo. O que mostrarei no que segue é uma abordagem bem mais modesta onde o problema da retroação é simplesmente desprezado e o espaço-tempo de fundo é dado a mão, o que dentro do escopo da TQCEC é um problema totalmente legítimo.

Considere o espaço-tempo $(\mathcal{M}, g)$ gerado por um sistema gravitacional que é estático no passado e que evolui suavemente até uma nova configuração, também estática, na qual ele permanece por algum tempo. Imagine, então, que esse sistema evolui novamente de modo suave até uma nova configuração estática no futuro. Assim como antes, suponha que sobre o espaço-tempo gerado por esse sistema está definido um campo escalar que obedece à Eq. (3.1.2). Esse sistema será construído de modo tal que na região estática intermediária do espaço-tempo em questão o campo passa por uma fase de instabilidades e seu vácuo é despertado.

Para computar o número de partículas produzidas numa fase ulterior ao despertar do vácuo do campo basta uma análise do comportamento temporal dos modos do campo nas regiões passada, intermediária e futura do espaço-tempo de fundo. Sendo assim, vou utilizar uma coordenada $t$ que cobre todo o espaço-tempo e que coincide, em cada uma das regiões do espaço-tempo, com o parâmetro temporal definido pela isometria temporal ali presente. Uma vez que as transições entre as regiões passada e intermediária e intermediária e futura de $(\mathcal{M}, g)$ são imaginadas serem suaves, é possível colar a coordenada temporal entre cada uma dessas regiões de sorte que $t: \mathcal{M} \rightarrow \mathbb{R}$ é uma função suave.

Vou considerar, então, quatro conjuntos distintos de modos da equação de campo. Os três primeiros, $\left\{u_{j}\right\}_{j \in \mathcal{I}},\left\{v_{l}\right\}_{l \in \mathcal{O}}$ e $\left\{w_{n}\right\}_{n \in \mathcal{O}^{\prime}}$ já foram construídos na Seção 5.1. Os modos $u_{j}$ são definidos por seu comportamento oscilatório no tempo no passado, enquanto que os modos $v_{l}$ e $w_{n}$ são definidos, respectivamente, por seu comportamento oscilatório e exponencial no tempo na região intermediária. Por fim, vou também considerar o conjunto 
de modos $\left\{p_{k}\right\}_{k \in \mathcal{U}}$, cujos elementos no futuro têm a forma

$$
p_{k}=\frac{e^{-i \vartheta_{k} t}}{\sqrt{2 \vartheta_{k}}} I_{k} .
$$

Suponha que o estado inicial do campo é o vácuo $\Psi_{0}^{\text {in }} \in \mathscr{F}_{\mathrm{s}}\left(\mathscr{H}_{\text {in }}\right)$, onde $\mathscr{H}_{\text {in }}$ é o espaço de Hilbert de uma partícula ao qual pertencem as soluções de freqüência positiva com respeito ao campo de Killing no passado. Desejo, então, calcular o valor esperado nesse estado do operador número associado a uma base de soluções de freqüência positiva no futuro. Como mostrado na Seção 8.1, essa quantidade está relacionada com o coeficiente $\beta$ de Bogoliubov, definido pela Eq. (3.3.12b), entre os modos $u_{j}$ e $p_{k}$. Nesse cálculo, contudo, é importante guardarmos a informação de que em algum momento as flutuações do campo passaram por uma fase de crescimento exponencial. Para tanto, vou primeiro definir os seguintes coeficientes da transformação de Bogoliubov entre as bases de modos consideradas:

$$
\begin{gathered}
p_{k}=\int_{\mathcal{O}} d \nu(l)\left(\chi_{k l} v_{l}-\bar{\lambda}_{k l} \bar{v}_{l}\right)+\sum_{n \in \mathcal{O}^{\prime}}\left(\chi_{k n} w_{n}-\bar{\lambda}_{k n} \bar{w}_{n}\right), \\
v_{l}=\int_{\mathcal{I}} d \mu(j)\left(\epsilon_{l j} u_{j}-\bar{\eta}_{l j} \bar{u}_{j}\right) \\
w_{n}=\int_{\mathcal{I}} d \mu(j)\left(\epsilon_{n j} u_{j}-\bar{\eta}_{n j} \bar{u}_{j}\right) \\
p_{k}=\int_{\mathcal{I}} d \mu(j)\left(\alpha_{k j} u_{j}-\bar{\beta}_{k j} \bar{u}_{j}\right) .
\end{gathered}
$$

Através da equação de campo é possível evoluir os modos $u_{j}$ até a região em que o mecanismo de despertar do vácuo é desengatilhado. Lá é conveniente reescrever os modos $u_{j}$ em termos dos modos $v_{l}$ e $w_{n}$ :

$$
u_{j}(t, \mathbf{x})=\int_{\mathcal{O}} d \nu(l)\left[\bar{\epsilon}_{l j} v_{l}(t, \mathbf{x})+\eta_{l j} \bar{v}_{l}(t, \mathbf{x})\right]+\sum_{n \in \mathcal{O}^{\prime}}\left[\bar{\epsilon}_{n j} w_{n}(t, \mathbf{x})+\eta_{n j} \bar{w}_{n}(t, \mathbf{x})\right]
$$

Aqui $\mathbf{x}$ simplesmente denota as coordenadas espaciais definidas sobre a hiperfície tipo espaço indexada pelo parâmetro $t$. Na região intermediária, $t$ foi identificado com a coordenada temporal construída sobre a isometria temporal ali presente. Considere agora uma translação temporal dos modos $u_{j}$ nessa região. Para os modos $v_{l}$ essa operação 
implica que

$$
v_{l}(t+T, \mathbf{x})=e^{-i \varpi_{l} T} v_{l}(t, \mathbf{x}),
$$

enquanto que para os modos $w_{n}$ ela leva a

$$
w_{n}(t+T, \mathbf{x})=\gamma_{\Omega_{n}}(T) w_{n}(t, \mathbf{x})-\bar{\theta}_{\Omega_{n}}(T) \bar{w}_{n}(t, \mathbf{x})
$$

A partir das definições da Eq. (3.3.12a) e da Eq. (3.3.12b), é possível calcular os coeficientes $\gamma_{\Omega_{n}}$ e $\theta_{\Omega_{n}}$ que comparecem na Eq. (8.3.5. São eles

$$
\gamma_{\Omega_{n}}(T)=-2 i \operatorname{senh}\left(\Omega_{n} T+i \pi / 6\right)
$$

e

$$
\theta_{\Omega_{n}}(T)=2 i \operatorname{senh}\left(\Omega_{n} T\right)
$$

A Eq. 8.3.5 é um reflexo do fato já apontado no Capítulo 7 de que a quantização do campo de KG, quando da presença da instabilidade, depende da escolha da superfície de Cauchy $\Sigma_{t}$ sobre a qual o produto interno do espaço de Hilbert de uma partícula é definido. A translação temporal dos modos $u_{j}$ dentro da região intermediária leva a

$$
\begin{aligned}
u_{j}(t+T, \mathbf{x})= & \int_{\mathcal{O}} d \nu(l)\left[\bar{\epsilon}_{l j} e^{-i \varpi_{l} T} v_{l}(t, \mathbf{x})+\eta_{l j} e^{i \varpi_{l} T} \bar{v}_{l}(t, \mathbf{x})\right] \\
& +\sum_{n \in \mathcal{O}^{\prime}}\left[\left(\bar{\epsilon}_{n j} \gamma_{\Omega_{n}}-\eta_{n j} \theta_{\Omega_{n}}\right) w_{n}(t, \mathbf{x})\right. \\
& \left.+\left(\eta_{n j} \bar{\gamma}_{\Omega_{n}}-\bar{\epsilon}_{n j} \bar{\theta}_{\Omega_{n}}\right) \bar{w}_{n}(t, \mathbf{x})\right] .
\end{aligned}
$$

Uma vez que os coeficientes de Bogoliubov Eq. (3.3.12) são conservados pela dinâmica, a forma acima para $u_{j}$ deve valer em qualquer região do espaço-tempo. Em particular, ela também deve ser verdadeira no passado, onde sabemos qual é a dependência temporal de $u_{j}$. Disso podemos inferir que a diferença entre $u_{j}(t, \mathbf{x})$ e $u_{j}(t+T, \mathbf{x})$ deve ser apenas uma fase. Então

$$
\begin{gathered}
u_{j}(t, \mathbf{x})=e^{i \varphi_{j}(T)} \int_{\mathcal{O}} d \nu(l)\left[\bar{\epsilon}_{l j} e^{-i \varpi_{l} T} v_{l}(t, \mathbf{x})+\eta_{l j} e^{i \varpi_{l} T} \bar{v}_{l}(t, \mathbf{x})\right] \\
+e^{i \varphi_{j}(T)} \sum_{n \in \mathcal{O}^{\prime}}\left[\left(\bar{\epsilon}_{n j} \gamma_{\Omega_{n}}-\eta_{n j} \theta_{\Omega_{n}}\right) w_{n}(t, \mathbf{x})\right. \\
\left.+\left(\eta_{n j} \bar{\gamma}_{\Omega_{n}}-\bar{\epsilon}_{n j} \bar{\theta}_{\Omega_{n}}\right) \bar{w}_{n}(t, \mathbf{x})\right] .
\end{gathered}
$$


Ao evoluir $u_{j}$ até o futuro, passa a ser conveniente expressar esses modos em termos da terceira base. Isso nos leva a

$$
\begin{gathered}
u_{j}=\int_{\mathcal{O}} d \nu(l) \int_{\mathcal{U}} d \varsigma(k)\left[e^{i \varphi_{j}(T)}\left(\bar{\epsilon}_{l j} \bar{\chi}_{k l} e^{-i \varpi_{l} T}+\eta_{l j} \chi_{k l} e^{i \varpi_{l} T}\right) p_{k}\right. \\
\left.+e^{i \varphi_{j}(T)}\left(\bar{\epsilon}_{l j} \lambda_{k l} e^{-i \varpi_{l} T}+\eta_{l j} \bar{\lambda}_{k l} e^{i \varpi_{l} T}\right) \bar{p}_{k}\right] \\
+\sum_{n \in \mathcal{O}^{\prime}} \int_{\mathcal{U}} d \varsigma(k)\left\{e ^ { i \varphi _ { j } ( T ) } \left[\left(\bar{\epsilon}_{n j} \gamma_{\Omega_{n}}-\eta_{n j} \theta_{\Omega_{n}}\right) \bar{\chi}_{k n}\right.\right. \\
\left.+\left(\eta_{n j} \bar{\gamma}_{\Omega_{n}}-\bar{\epsilon}_{n j} \bar{\theta}_{\Omega_{n}}\right) \chi_{k n}\right] p_{k} \\
e^{i \varphi_{j}(T)}\left[\left(\bar{\epsilon}_{n j} \gamma_{\Omega_{n}}-\eta_{n j} \theta_{\Omega_{n}}\right) \lambda_{k n}\right. \\
\left.\left.+\left(\eta_{n j} \bar{\gamma}_{\Omega_{n}}-\bar{\epsilon}_{n j} \bar{\theta}_{\Omega_{n}}\right) \bar{\lambda}_{k n}\right] \bar{p}_{k}\right\}
\end{gathered}
$$

Por outro lado, em termos dos modos $p_{k}$, os modos $u_{j}$ se escrevem como

$$
u_{j}=\int_{\mathcal{U}} d \varsigma(k)\left(\bar{\alpha}_{k j} p_{l}+\beta_{k j} \bar{p}_{l}\right)
$$

Comparando essas duas expressões, vem que

$$
\begin{aligned}
\beta_{k j}= & e^{i \varphi_{j}(T)} \int_{\mathcal{O}} d \nu(l)\left(\bar{\epsilon}_{l j} \lambda_{k l} e^{-i \varpi_{l} T}+\eta_{l j} \bar{\lambda}_{k l} e^{i \varpi_{l} T}\right) \\
& +e^{i \varphi_{j}(T)} \sum_{n \in \mathcal{O}^{\prime}}\left[\left(\bar{\epsilon}_{n j} \gamma_{\Omega_{n}}-\eta_{n j} \theta_{\Omega_{n}}\right) \lambda_{k n}+\left(\eta_{n j} \bar{\gamma}_{\Omega_{n}}-\bar{\epsilon}_{n j} \bar{\theta}_{\Omega_{n}}\right) \bar{\lambda}_{k n}\right] .
\end{aligned}
$$

A fórmula acima mostra que o número de partículas criadas quando o sistema retorna ao equilíbrio no futuro cresce exponencialmente com o tempo durante o qual o mecanismo de despertar do vácuo atua. Logo, o "adormecimento" do vácuo leva a um aumento da quantidade de energia estocada nos graus de liberdade do campo. Note que enquanto o vácuo está "desperto" a energia total do campo é conservada, mesmo havendo um crescimento exponencial da densidade de energia. Portanto, durante esse processo a energia é rearranjada ao longo da seção espacial, mas não injetada no campo por qualquer mecanismo externo que seja. Assim como já apontado na Seção 5.4. esse rearranjo dá-se de sorte que em algumas regiões da seção espacial a densidade de energia fica cada vez mais positiva, enquanto que em outras ela fica cada vez mais negativa. Contudo, quando o sistema retorna ao equilíbrio no futuro, todas as partículas criadas têm energia positiva. Nesse cenário, como é possível dar conta da conservação da energia do sistema gravitacional mais campo quântico? 
A despeito do que foi mencionado acima, sabemos que não podemos questionar o fato de que $\nabla^{a}\left\langle\hat{T}_{a b}\right\rangle=0$. Seja $\mathcal{N} \subset \mathcal{M}$ uma porção da variedade $\mathcal{M}$ compreendida entre duas superfícies de Cauchy $\Sigma_{t_{\mathrm{i}}}$ e $\Sigma_{t_{\mathrm{f}}}$, sendo que $\Sigma_{t_{\mathrm{f}}}$ está contido no domínio de dependência futuro de $\Sigma_{t_{\mathrm{i}}}$. Seja $n^{a}$ um campo vetorial tipo tempo normalizado e que é ortogonal à foliação escolhida para o espaço-tempo $(\mathcal{N}, g)$ e, em particular, às hiperfícies $\Sigma_{t_{\mathrm{i}}}$ e $\Sigma_{t_{\mathrm{f}}}$. Seja $v^{a}$ um outro campo vetorial tipo tempo. É claro que $v^{b} \nabla^{a}\left\langle\hat{T}_{a b}\right\rangle=0$. Integrando essa identidade em toda a subvariedade $\mathcal{N}$ e aplicando o teorema de Stokes, vem que

$$
\int_{\Sigma_{t_{\mathrm{f}}}} d \Sigma n^{a} v^{b}\left\langle\hat{T}_{a b}\right\rangle-\int_{\Sigma_{t_{\mathrm{i}}}} d \Sigma n^{a} v^{b}\left\langle\hat{T}_{a b}\right\rangle-\int_{\mathcal{N}} \sqrt{-g} d^{4} x \nabla^{a} v^{b}\left\langle\hat{T}_{a b}\right\rangle=0
$$

Esse resultado sugere a definição do seguinte tensor:

$$
w_{a b}:=\nabla_{a} v_{b}+\nabla_{b} v_{a}
$$

que deve depender somente da métrica $g_{a b}$ e do campo $v^{a}$. Com o tensor $w_{a b}$ posso reescrever a expressão integrada para a conservação do valor esperado do tensor energiamomentum do campo como

$$
\int_{\Sigma_{t_{\mathrm{f}}}} d \Sigma n^{a} v^{b}\left\langle\hat{T}_{a b}\right\rangle-\int_{\Sigma_{t_{\mathrm{i}}}} d \Sigma n^{a} v^{b}\left\langle\hat{T}_{a b}\right\rangle=\frac{1}{2} \int_{\mathcal{N}} \sqrt{-g} d^{4} x w^{a b}\left\langle\hat{T}_{a b}\right\rangle
$$

Suponha que os vetores $\left.\frac{v^{a}}{\|v\|}\right|_{p}$ e $\left.\frac{v^{a}}{\|v\|}\right|_{q}$, com $p \in \Sigma_{t_{\mathrm{i}}}$ e $q \in \Sigma_{t_{\mathrm{f}}}$, são ortogonais respectivamente às hiperfícies $\Sigma_{t_{\mathrm{i}}}$ e $\Sigma_{t_{\mathrm{f}}}$. Considere, então, um observador cuja linha de mundo passa pelos pontos $p$ e $q$ e que nesses pontos tem como seção espacial as hiperfícies $\Sigma_{t_{\mathrm{i}}}$ e $\Sigma_{t_{\mathrm{f}}}$. Desse modo, do ponto de vista do observador, a diferença do lado esquerdo na Eq. (8.3.16) tem a interpretação de ser a variação do valor esperado da energia total do campo entre os instantes definidos pelas hiperfícies $\Sigma_{t_{\mathrm{i}}}$ e $\Sigma_{t_{\mathrm{f}}}$. No lado direito da Eq. 8.3.16), o tensor $w_{a b}$ pode ser visto como uma medida de quanto $v^{a}$ falha em ser um campo de Killing tipo tempo. Por conta da interpretação do lado esquerdo dessa equação, o lado direito pode ser visto como o trabalho feito pelo campo gravitacional sobre o campo quântico do ponto de vista desse observador hipotético.

Pois bem. Suponha agora que a hiperfície $\Sigma_{t_{\mathrm{i}}}$ corresponda ao instante de tempo $t_{\mathrm{i}}$ como medido por um observador que segue alguma linha integral do campo de Killing 
tipo tempo da região intermediária e que o campo vetorial $v^{a}$ coincide com esse campo de Killing. Se a hiperfície $\Sigma_{t_{\mathrm{f}}}$ descreve a seção espacial desse observador no futuro, quando o sistema gravitacional mais o campo quântico encontram-se em um novo estado estacionário, então, do ponto de vista desse observador, a Eq. 8.3.16 dá conta do balanço entre a variação de energia do campo quântico e o trabalho realizado sobre ele pelo campo gravitacional. Sendo assim, o fato de que durante a existência da instabilidade o valor esperado do tensor energia-momentum no vácuo cresce exponencialmente implica que o trabalho realizado pelo campo gravitacional sobre o campo quântico contem uma contribuição que foi exponencialmente amplificada. Isso mostra que a imensa produção de energia na forma de partículas do campo quando o vácuo adormece ocorre às expensas da energia do próprio sistema gravitacional. Note ainda que, como o crescimento exponencial das flutuações quânticas independe do quão lentamente se dá a mudança da geometria de fundo, a criação de partículas por conta do "adormecimento" do vácuo guarda diferenças com relação ao mecanismo usual de produção espontânea de partículas em TQCEC.

A fim de ilustrar o que foi dito acima, exibirei aqui um modelo bastante simples no qual é possível calcular explicitamente os coeficientes da transformação de Bogoliubov. Considere exatamente o cenário apresentado nesta seção. Suponha que em alguma região do espaço é colocada uma pequena caixa cúbica cujo lado tem, assintoticamente no passado, tamanho próprio $L$ e com paredes capazes de blindar o campo quântico. A caixa deve ser suficientemente pequena para que o campo ali confinado não seja sensível à curvatura do espaço-tempo nas direções espaciais. Assim, a equação de campo pode ser colocada na seguinte forma:

$$
\frac{\partial^{2}}{\partial t^{2}} \phi-\nabla^{2} \phi+V_{\mathrm{ef}}(t) \phi=0
$$

onde $\nabla^{2}$ denota o operador laplaciano usual e $V_{\text {ef }}$ é um potencial efetivo que decorre da variação temporal das quantidades geométricas na região interior à caixa. Para essa equação escolherei a seguinte forma para os modos:

$$
u_{\mathbf{n}}(t, x, y, z)=f_{\mathbf{n}}(t)\left(\frac{2}{L}\right)^{3 / 2} \operatorname{sen}\left(\frac{n_{x} \pi x}{L}\right) \operatorname{sen}\left(\frac{n_{y} \pi y}{L}\right) \operatorname{sen}\left(\frac{n_{z} \pi z}{L}\right)
$$


onde $\mathbf{n}=\left(n_{x}, n_{y}, n_{z}\right)$ e $n_{x}, n_{y}, n_{z} \in \mathbb{N}^{*}$. A função $f_{\mathbf{n}}(t)$ satisfaz

$$
\frac{d^{2}}{d t^{2}} f_{\mathbf{n}}(t)+\left[\sigma+V_{\mathrm{ef}}(t)\right] f_{\mathbf{n}}(t)=0
$$

A constante $\sigma$ aparece por conta da separação de variáveis e sua relação com $n_{x}, n_{y}$ e $n_{z}$ é

$$
\sigma=\left(\frac{n_{x} \pi}{L}\right)^{2}+\left(\frac{n_{y} \pi}{L}\right)^{2}+\left(\frac{n_{z} \pi}{L}\right)^{2}
$$

No passado o espaço-tempo é estático, o que implica que $V_{\text {ef }}(t \rightarrow-\infty)=V_{\text {in }}$. Isso permite que a base $\left\{u_{\mathbf{n}}\right\}_{\mathbf{n} \in \mathbb{N}^{* 3}}$ seja escolhida de maneira que para $t \rightarrow-\infty$

$$
f_{\mathbf{n}}(t)=\frac{e^{-i \omega_{\mathbf{n}} t}}{\sqrt{2 \omega_{\mathbf{n}}}}
$$

sendo que $\omega_{\mathbf{n}}:=\sqrt{\sigma+V_{\text {in }}}$. Assintoticamente no futuro teremos que $V_{\text {ef }}(t \rightarrow+\infty)=V_{\text {out }}$, o que permite a construção dos modos

$$
p_{\mathbf{n}}(t, x, y, z)=g_{\mathbf{n}}(t)\left(\frac{2}{L}\right)^{3 / 2} \operatorname{sen}\left(\frac{n_{x} \pi x}{L}\right) \operatorname{sen}\left(\frac{n_{y} \pi y}{L}\right) \operatorname{sen}\left(\frac{n_{z} \pi z}{L}\right)
$$

de forma que quando $t \rightarrow+\infty$ temos

$$
g_{\mathbf{n}}(t)=\frac{e^{-i \vartheta_{\mathbf{n}} t}}{\sqrt{2 \vartheta_{\mathbf{n}}}}
$$

onde $\vartheta_{\mathbf{n}}:=\sqrt{\sigma+V_{\text {out }}}$. Disso e da definição do coeficiente $\beta$ de Bogoliubov, Eq. 3.3.12b, segue que

$$
\beta_{\left(n_{x}, n_{y}, n_{z}\right)}:=\beta_{\mathbf{n}, \mathbf{m}}=i \Omega\left(\bar{u}_{\mathbf{n}}, \bar{p}_{\mathbf{m}}\right)=i\left(\bar{g}_{\mathbf{m}} \frac{d}{d t} \bar{f}_{\mathbf{n}}-\bar{f}_{\mathbf{n}} \frac{d}{d t} \bar{g}_{\mathbf{m}}\right) \delta_{\mathbf{n}, \mathbf{m}}
$$

Para calcular $\beta_{\mathbf{n}, \mathbf{m}}$ podemos evoluir os modos $u_{\mathbf{n}}$ até o futuro e lá projetá-los nos modos $\bar{p}_{\mathbf{m}}$. A título de exemplo, vou tomar a seguinte forma para $V_{\mathrm{ef}}(t)$ :

$$
V_{\mathrm{ef}}(t)=\frac{V_{0}}{2} \frac{\tanh [c(t+T / 2)]-\tanh [c(t-T / 2)]}{\tanh [c T / 2]} .
$$

Por simplicidade, escolhi os parâmetros de $V_{\text {ef }}$ de maneira que somente o modo $u_{(1,1,1)}$ experimente o crescimento exponencial. Para esse potencial efetivo a tarefa de evoluir os modos $u_{\mathbf{n}}$ só pode ser feita numericamente. A Fig. 8.1 ilustra o perfil de "bacia" do 


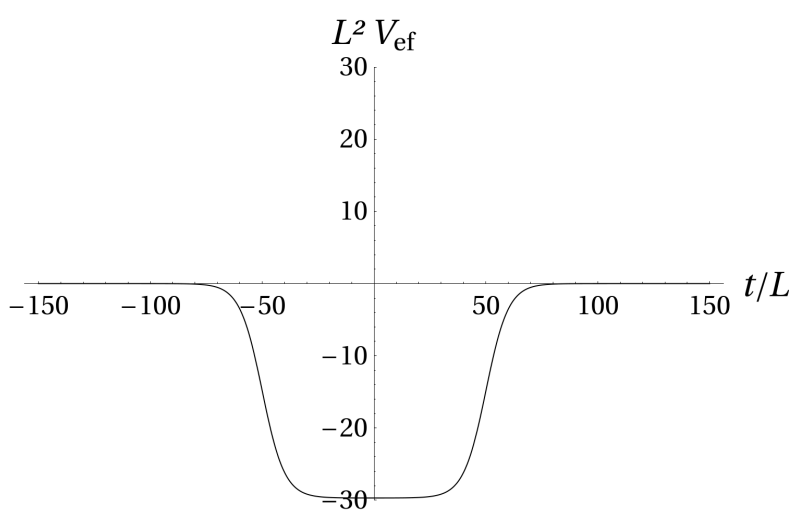

Figura 8.1 - Perfil do potencial efetivo dado na Eq. 8.3.25 para os seguintes valores dos parâmetros: $c=0.1 / L, T=100 L$ e $V_{0}=-29.7 / L^{2}$.

potencial efetivo utilizado. Os gráficos na Fig. 8.2 verificam o comportamento exponencial de $\beta_{(1,1,1)}$. Na Fig. 8.2(a) está apresentado o comportamento de $\ln \left|\beta_{(1,1,1)}\right|^{2}$ em função do parâmetro $T$, fixado $V_{0}$ e $c$, enquanto que na Fig. 8.2(b) tem-se o mesmo gráfico, mas com $V_{0}$ e $c T$ fixos.

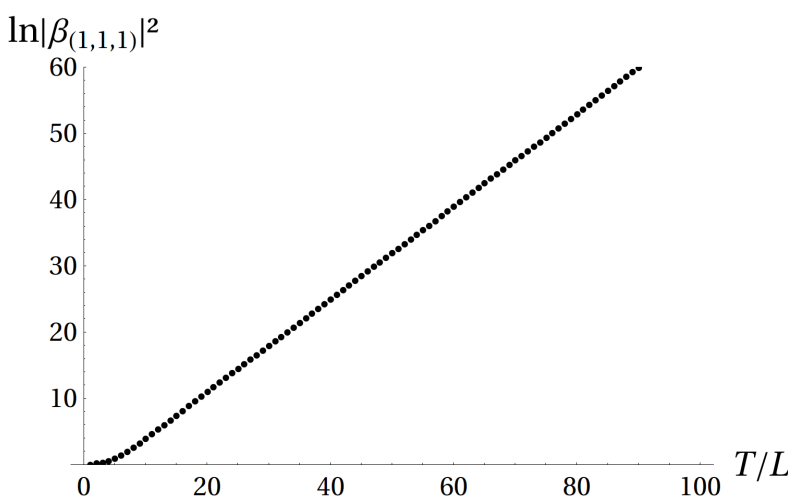

(a)

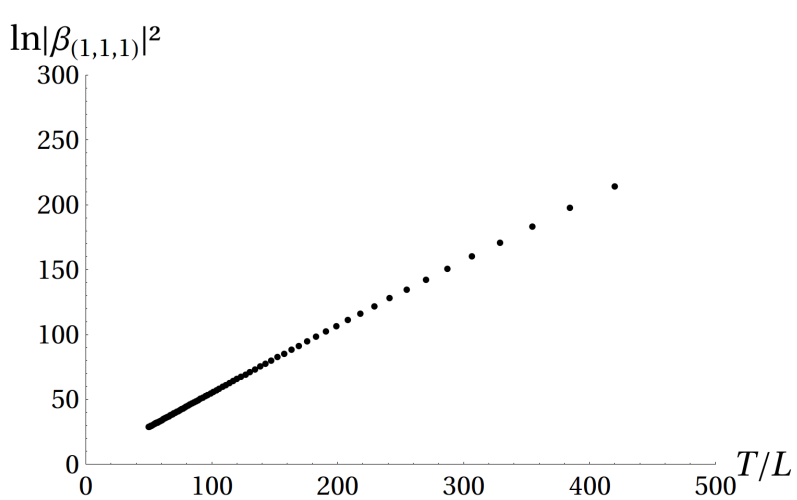

(b)

Figura 8.2 - Gráfico do comportamento de $\ln \left|\beta_{(1,1,1)}\right|^{2}$ em função de $T$. Em (a) foi fixado $c=0.1 / L$ e $V_{0}=-29.7 / L^{2}$, enquanto que em (b) temos $c T=100, V_{0}=-29.7 / L^{2}$.

Dentro do contexto desse simples modelo podemos novamente nos inquirir quanto a questão da conservação de energia. Aqui, todavia, nada sabemos do tensor métrico e, conseqüentemente, do tensor energia-momentum associado ao campo. Não obstante, é possível, manipulando a Eq. 8.3.17), construir uma equação de conservação. De fato, multiplicando a Eq. 8.3.17 por $\partial_{t} \bar{\phi}$ e somando o resultado com seu complexo conjugado vem, após alguma manipulação das derivadas, o seguinte:

$$
\frac{\partial}{\partial t}\left(\left|\frac{\partial \phi}{\partial t}\right|^{2}+V_{\mathrm{ef}}|\phi|^{2}+|\nabla \phi|^{2}\right)-\nabla \cdot\left(\frac{\partial \bar{\phi}}{\partial t} \nabla \phi+\frac{\partial \phi}{\partial t} \nabla \bar{\phi}\right)=\frac{d}{d t} V_{\mathrm{ef}}|\phi|^{2}
$$


Daí vê-se que, assim como antes, o fato de o sistema gravitacional - aqui descrito por $V_{\text {ef }}$ - sair do estado que permite a existência do mecanismo de despertar do vácuo e o crescimento exponencial das flutuações do campo são, juntos, os responsáveis pelo crescimento da energia do sistema quântico quando o sistema retorna ao equilíbrio. Isso mostra que o custo energético para a variação do potencial efetivo de modo a despertar o vácuo é desprezível quando comparado ao custo energético necessário para variá-lo depois que as flutuações do vácuo foram exponencialmente amplificadas.

Como já mencionado no início desta seção, aqui em momento algum toquei na questão da retroação do campo quântico. A energia injetada no campo ao longo de todo o processo e, em particular, ao final da fase durante a qual atua o mecanismo de despertar do vácuo é transformada, no futuro, em partículas. Ora, é de se esperar que, quando levada em conta, a retroação do campo use parte dessa energia que vai sendo injetada no campo para modificar o sistema gravitacional. Somente uma fração, então, seria liberada na forma de partículas. Um corpo com massa da ordem da do Sol, aproximadamente $10^{30} \mathrm{~kg}$, que seja capaz de desengatilhar o efeito deve acumular numa região da ordem do seu raio ( $10 \mathrm{~km}$ ) uma energia da ordem de $10^{54} \mathrm{TeV}$ para que o campo gravitacional gerado pelo vácuo rivalize com o gerado pela matéria clássica. Dentro da escala dos fenômenos de altas energias essa é uma energia tão grande que mesmo que somente uma pequena fração fosse convertida em partículas ainda assim teríamos um evento de imensas proporções. Essa "componente escura", haja visto que essas partículas interagem exclusivamente com a gravitação, produzida quando o vácuo adormece seria responsável por carregar para longe parte da energia inicialmente armazenada no sistema clássico. 


\section{Capítulo 9}

\section{Questões pertinentes}

$\mathrm{O}$

S cenários exibidos nos últimos quatro capítulos e a partir dos quais argumentei em favor do efeito envolvem espaços-tempos que são ao menos assintoticamente estáticos tanto no passado quanto no futuro. Além disso, no Capítulo 5 me restringi a espaços-tempos conformalmente estáticos. É importante que se diga, contudo, que a existência do efeito e suas possíveis implicações para a física macroscópica de sistemas gravitacionais clássicos não devem depender dessas hipóteses. Tais simplificações foram feitas somente com o intuito de tornar a linha argumentativa percorrida mais contundente. De fato, esperamos que o efeito, justamente por já se fazer presente em situações por vezes tão simplificadas, seja desengatilhado em sistemas gravitacionais mais complexos. Também aqui fizemos sempre uso de um campo escalar com massa e acoplamento não-mínimo satisfazendo a equação de KG Eq. 3.1.2. É bem verdade que não se conhece na Natureza nenhuma partícula fundamental que seja descrita por um campo escalar ${ }^{1}$ Todavia, é bem-sabido que desconhecemos a natureza de aproximadamente $95 \%$ do conteúdo de energia do nosso Universo e que, aparentemente, esse conteúdo só é acessível gravitacionalmente. Logo, alternativas para se testar a existência de campos livres na Natureza, como o Efeito de Despertar do Vácuo, são muito bem-vindas.

A hipótese de estaticidade durante a fase em que o vácuo encontra-se "desperto" im-

\footnotetext{
${ }^{1}$ Mésons são descritos por campos escalares, mas são formados por um par quark-antiquark. Dentro do Modelo Padrão das partículas elementares a partícula de Higgs seria descrita por um campo escalar, mas até o momento o CERN continua a sua procura.
} 
plica, por conta da conservação do valor esperado do tensor energia-momentum associado ao campo quântico, que sua energia total é conservada.2 Essa afirmação foi verificada na Seção 5.4 a despeito do crescimento exponencial dos modos $w_{n}$. Ademais, verificamos que tanto o tensor energia-momentum associado a cada modo $w_{n}$ quanto o valor esperado do tensor energia-momentum no vácuo inicial na região em que o efeito é disparado são covariantemente conservados. Vale notar ainda que a forma dos modos $w_{n}$ na Eq. 5.1 .22 e o fato de $\left\langle\hat{T}_{0 i}\right\rangle \neq 0$ na Eq. $5.2 .2 \mathrm{~b}$ vai ao encontro de que foi argumentado no Capítulo 7 a respeito da inexistência de um operador atuando sobre o espaço de estados do campo quântico que implemente a isometria temporal do espaço-tempo de fundo.

Ao longo de praticamente toda esta tese me referi ao fenômeno aqui apresentado como um efeito de despertar do vácuo; um fenômeno de Teoria Quântica de Campos. Por outro lado, na Eq. (5.3.3), onde estimo a ordem de grandeza de $\bar{\Omega}$, não há menção a $\hbar$. Se $\hbar$ é a impressão digital do mundo quântico, então em que ponto, nos cálculos, a constante de Planck aparece? Se recuperarmos as unidades em que é usualmente escrito, o comutador entre o operador campo e seu momentum canonicamente conjugado é proporcional a $\hbar$. Isso, juntamente com o fato de que a variável temporal $t$ sempre tem um fator $c$ multiplicando, implica que na frente de todos os modos vai um fator $\sqrt{\hbar c}$. Esse fator dá a ordem de grandeza das amplitudes das flutuações do vácuo. Logo, a Mecânica Quântica participa de forma crucial do efeito na medida em que permite de forma incontornável que todo tipo de flutuação esteja presente no estado inicial do campo. Em particular, contempla aquelas flutuações que experimentarão crescimento sem-limites quando o vácuo é "despertado", crescimento este que acaba se manifestando no tensor energia-momentum do campo e tomando conta da evolução do sistema.

Acredito que aqui ainda cabe um pequeno comentário quanto a renormalização do valor esperado de $\hat{T}_{a b}$ quando da presença do mecanismo de despertar do vácuo. Na Seção 5.3, fraseei o mecanismo em termos de um potencial efetivo negativo. Como mencionado, o fato de o potencial efetivo ficar negativo não é capaz, por si, de garantir a

\footnotetext{
${ }^{2}$ Isso é verdade enquanto não consideramos a retroação do campo quântico sobre o espaço-tempo de fundo.
} 
existência de modos da equação de campo cuja parte temporal cresce exponencialmente: é necessário que esse potencial efetivo seja negativo o suficiente em uma região grande o suficiente. Isso deixa claro que o efeito aqui apresentado depende necessariamente de propriedades não-locais do espaço-tempo. Há ainda outra característica do mecanismo não explicitamente mencionada no Capítulo 5; sua relação com condições iniciais. Na Teoria Quântica de Campos, as flutuações presentes em qualquer estado inevitavelmente acabam por contemplar toda sorte de perfis de campo consistentes com as condições de contorno do sistema. Dentre esses perfis sempre haverá aqueles que têm projeção nãonula nos modos que são assintoticamente divergentes. Por um lado, então, o Efeito de Despertar do Vácuo é robusto com relação às condições iniciais, haja visto a presença de flutuações em qualquer que seja o estado inicial escolhido. Por outro, os possíveis estados podem combinar de modo diferente aquelas flutuações, o que implica, no futuro, que os valores esperados dos observáveis relativos ao campo nos diferentes estados iniciais sejam diferentes. À luz do que já foi mencionado ao final do Capítulo 3 quanto a independência de qualquer procedimento de renormalização em relação ao estado do campo, essas duas características do efeito nos levam a concluir que os procedimentos de regularização e renormalização que podem ser usados para dar sentido a $\left\langle\hat{T}_{a b}\right\rangle$ não podem ser capazes de inviabilizar o mecanismo. É importante frisar que, no contexto da renormalização via separação de pontos, o efeito está em acordo com os teoremas que garantem que a evolução do sistema pela equação de campo em um espaço-tempo globalmente hiperbólico não é capaz de promover novas divergências além daquelas já renormalizadas $(6062)$. Com efeito, uma vez que, a despeito do crescimento sem-limites das flutuações do campo, no caso exibido no Capítulo 5. $\left\langle\hat{\phi}^{2}\right\rangle$ é livre de singularidades, pois, a rigor, somente diverge assintoticamente no futuro.

Da maneira como foi fraseado no Capítulo 5, o mecanismo de despertar do vácuo é revelado quando a Eq. 3.1.2 admite soluções assintoticamente no futuro com $\sigma<0$. Em última instância, esse fato depende exclusivamente da equação de campo e de condições de contorno. Logo, esse crescer sem-limites de perfis do campo no futuro são instabilidades que já se fazem presentes na teoria clássica. Não é uma exclusividade da versão 
quantizada do campo. Portanto, em princípio, mesmo o efeito sendo disparado para as flutuações do campo quântico, ele não seria capaz de sobrepujar qualquer densidade de energia clássica, uma vez que configurações clássicas do campo poderiam compor o sistema e eventualmente crescerem pelo menos tanto quanto as flutuações de vácuo. Esse quadro seria simples assim se pudéssemos desconsiderar o papel de fontes e interações na teoria. Em primeiro lugar, para ser possível construir uma configuração clássica para o campo seria necessário fazê-lo via alguma fonte para a Eq. (3.1.2), coisa que não consideramos. Em nosso estudo somente comparecem amplitudes decorrentes das flutuações de vácuo. Em segundo lugar, não necessariamente todas as condições iniciais possíveis para essas configurações clássicas implicarão num crescimento da amplitude do campo. Em contrapartida, como já mencionado acima, no estado de vácuo estão contemplados todos as possíveis configurações de campo consistentes com as condições de contorno. Logo, na versão quântica, o efeito é inevitavelmente disparado. Por último, consideramos só de passagem no Capítulo 8 a possibilidade de o campo interagir com outros sistemas. Essa é uma questão particularmente interessante. Muito embora as flutuações do campo quântico no vácuo cresçam violentamente durante a atuação do efeito, o valor esperado de sua amplitude é sempre nulo. Por outro lado, se configurações clássicas de $\phi$ sofrerem a amplificação promovida pelo efeito, sua amplitude irá crescer. Uma vez acoplado com outro campo - como no caso de uma eletrodinâmica escalar, por exemplo — é forçoso que $\phi$, antes mesmo de desempenhar papel gravitacional relevante, produza pares que possivelmente retroagirão de modo a minimizar o efeito desse campo clássico.

Em um artigo recente, P. Pani et al. estudaram uma outra possível relação que poderia haver entre o mecanismo de despertar do vácuo e um perfil clássico não-nulo para o campo $\phi(63)$. Nesse trabalho os autores especulam que o Efeito de Despertar do Vácuo poderia induzir um fenômeno conhecido na literatura por "Escalarização Espontânea" 64, 65). No contexto da Escalarização Espontânea, o campo gravitacional é descrito por um tensor métrico, familiar à RG, e por campos escalares sem massa que se acoplam com os campos de matéria. A motivação para o estudo dessa descrição do campo gravitacional, alternativa à RG e conhecida na literatura como Teorias Tensor-escalar, está baseada no 
fato de que algumas teorias de unificação entre a Gravitação e as outras forças dão origem a campos escalares sem massa acoplados gravitacionalmente aos campos de matéria. Teorias Tensor-escalar podem ser mapeadas, mediante uma transformação conforme adequada, nas chamadas Teorias $f(R)$, teorias também alternativas à $\mathrm{RG}$ onde a lagrangiana do campo gravitacional é alguma função do escalar de curvatura (para uma revisão sobre o assunto, veja, e.g. a referência (66)). Testes para a RG no regime de campo gravitacional fraco impõem limites sobre o acoplamento entre os campos escalares e os de matéria, implicando, nesse regime, em desvios pequenos em relação à gravitação einsteiniana. Para campos gravitacionais intensos, como o de estrelas de nêutrons, entretanto, T. Damour e G. Esposito-Farèse argumentam na referência (64) que, mesmo quando os limites impostos pelos testes em campo fraco são satisfeitos, existem soluções auto-consistentes estáticas e esfericamente simétricas estáveis onde o campo escalar tem perfil não-nulo. Esse perfil não-nulo leva a uma mudança da massa gravitacional da estrela que pode chegar a uma fração considerável em relação ao que seria esperado pela RG. Logo, mesmo em acordo com os limites impostos pelos testes em campo fraco, Teorias Tensor-escalar, no regime de campo forte, levam a desvios significativos em relação às previsões da RG. Na referência (65) os mesmos autores argumentam ainda que o aparecimento de um perfil não-nulo para o campo escalar, a Escalarização Espontânea, guarda semelhanças com a magnetização de um material ferromagnético abaixo da temperatura de Curie. Ainda percorrendo alguns trabalhos relacionados a esse fenômeno, descobrimos que em 1997 T. Harada já tinha encontrado as instabilidades por nós apresentadas na referência (25) ao estudar a estabilidade dessas Teorias Tensor-escalar no contexto de estrelas (67). Harada, contudo, não fez qualquer conexão entre seu resultado e questões relativas à TQCEC. Pois bem. O que Pani et al. mostraram no trabalho da referência (63) é que, para corpos compactos, os valores de $M / \mathrm{R}$ e $\xi$ para os quais o vácuo é despertado são exatamente os mesmos necessários para o aparecimento da Escalarização Espontânea. Isso certamente levanta questionamentos interessantes acerca da relação entre a Escalarização Espontânea e o mecanismo por nós apresentado. Há, no entanto, duas diferenças importantes entre esse resultado e os que por nós foram apresentados, particularmente no trabalho mencionado 
na referência (25). Em primeiro lugar, os diagramas apresentados na figura 1 da referência (63) correspondem à existência de soluções autoconsistentes da equação de Einstein e da equação de KG com acoplamento não-mínimo com o escalar de curvatura. No nosso caso, a geometria é dada pela equação de Einstein e o campo quântico evolui sobre esse espaço-tempo de fundo. A segunda diferença é que enquanto a Escalarização Espontânea consiste em soluções que apresentam um perfil clássico não-nulo para o campo escalar, no Efeito de Despertar do Vácuo o perfil médio do campo permanece nulo mesmo quando suas flutuações crescem exponencialmente. A explicação para o resultado de Pani et al. pode ser traçada de volta na equação para o campo escalar, que é a mesma no dois casos. Em particular, a coincidência entre as fronteiras das regiões preenchidas no diagrama da figura 6.1(a) e no diagrama da esquerda da figura 1 da referência (63) pode ser explicada notando-se que nos dois casos essa linha demarca o limiar onde a solução da equação de KG é nula. Nesse caso as equações por nós examinadas, que não levam em conta a retroação do campo quântico, e as utilizadas no estudo de Pani et al., que consideram a retroação do campo clássico, coincidem. A conclusão que Pani et al. tiram de seu resultado é que ele poderia indicar que a retroação do mecanismo de despertar do vácuo sobre o espaço-tempo de fundo poderia dirigir a evolução do sistema gravitacional de modo a levá-lo a um estado de equilíbrio no qual apareceria a Escalarização Espontânea.

De fato, é de se esperar que a não-linearidade induzida na equação satisfeita pelo campo devido a retroação deste sobre o espaço-tempo de fundo aponte para um novo vácuo que seja estável. Quiçá esse novo vácuo corresponda a um perfil clássico do campo não-nulo que, talvez, seja aquele esperado pela Escalarização Espontânea

A essa especulação, todavia, é possível dirigir algumas críticas. A primeira é quanto à dinâmica do sistema gravitacional. Nas referências $(63 \sqrt[65)]{6}$, para sustentar que existem soluções para o sistema de equações com campo escalar não-nulo que são mais favoráveis do que as com campo escalar nulo, é mostrado que as últimas têm uma energia de ligação gravitacional menor do que as primeiras para os mesmos valores dos parâmetros. Em momento algum é abordado o problema do ponto de vista dinâmico, ou seja, se dadas condições iniciais e de contorno o sistema evoluirá para uma configuração de equilíbrio 
na qual um perfil não-nulo para o campo $\phi$ é sustentado. A segunda objeção diz respeito ao processo de retroação do campo escalar. Mesmo que para o caso puramente clássico fosse mostrado que a dinâmica auto-consistente da métrica do espaço-tempo e do campo escalar levasse o sistema gravitacional a um estado final no qual o fenômeno de Escalarização Espontânea desempenha algum papel, isso não implica que o mesmo aconteceria para a dinâmica semiclássica. De fato, se escrevermos o perfil clássico em termos dos elementos de uma base completa para as soluções da equação de campo, é possível verificar que a expressão para o tensor energia-momentum clássico é diferente da expressão para o valor esperado da versão quântica dessa quantidade, por exemplo, em um estado coerente que reproduza o perfil clássico do campo. Isso acontece, pois, em contraposição ao caso clássico, o valor esperado do tensor energia-momentum está ligado às flutuações do campo e não a seu perfil médio. Daí, então, a conclusão de que o processo de evolução autoconsistente do sistema clássico e do semiclássico devem, a princípio, ser diferentes. Talvez uma boa ilustração disso seja o caso em que campo está em seu estado de vácuo. No caso clássico, isso corresponde à condição inicial do campo e seu momentum canonicamente conjugado nulos, configuração essa que a evolução perpetuará. No caso semiclássico, a despeito de no estado de vácuo o perfil do campo e de seu momentum canonicamente conjugado serem nulos, o mesmo não é verdade para as flutuações. Como já argumentado nos capítulos anteriores, se temos que o vácuo é despertado, essas flutuações podem influenciar dramaticamente a evolução do sistema gravitacional. De qualquer maneira, a despeito dos questionamentos que aqui levanto, a possibilidade de uma relação entre o fenômeno de Escalarização Espontânea e o despertar do vácuo, como aventada na referência (63), só pode ser confirmada ou descartada quando for possível levar em conta de modo apropriado a retroação do campo escalar sobre o espaço-tempo de fundo.

Em espaço-tempo plano, o problema de como quantizar o campo de KG quando existem modos com freqüência imaginária já aparece em um trabalho do ano de 1940 assinado por L. I. Schiff, H. Snyder e J. Weinberg (68). Nele os autores mostram que o aparecimento desses modos é possível para um campo escalar carregado sob a ação de um potencial elétrico estacionário suficientemente profundo o qual, a título de exemplo, 
é tomado como sendo um poço quadrado. O problema da quantização do campo escalar quando da presença da instabilidade de Schiff, Snyder e Weinberg foi retomado e aprofundado trinta anos mais tarde por B. Schröer e J. A. Swieca na referência (69). A meu ver há dois aspectos interessantes sobre o estudo realizado por Schröer e Swieca que merecem menção. Em primeiro lugar, é afirmado logo na introdução que, do ponto de vista físico, a instabilidade de Schiff, Snyder e Weinberg não implica em uma instabilidade catastrófica e que, portanto, não haveria nenhum motivo a priori para descartá-la. Os autores, no entanto, somente dão atenção a energia e carga totais do campo, quantidades estas conservadas pela dinâmica, e não a quantidades locais, como a densidade de energia do campo. O segundo aspecto interessante é a discussão acerca da representação das relações canônicas de comutação do campo. Schröer e Swieca propõem duas representações distintas: a primeira em termos de um espaço que se assemelha a um de Fock, mas com norma indefinida, enquanto que a segunda é construída na mesma linha do que foi exposto na Seção 7.2 desta tese e que foi por nós reencontrada de maneira independente. Vale ainda mencionar que o estudo da instabilidade descoberta por Schiff, Snyder e Weinberg foi ainda aprofundado por S. A. Fulling na referência $(70) \cdot \mathrm{H}^{3}$

O estudo de instabilidades em TQCEC promovidas pela evolução do espaço-tempo de fundo já aparece, em um contexto diferente daquele abordado nesta tese, em trabalhos de meados da década de $1980(71,74)$ 年 O foco desses trabalhos é o aparecimento de instabilidades em um campo escalar com uma auto-interação $\lambda \phi^{4}$ induzida por flutuações quânticas no espaço-tempo de de Sitter. Ali é mostrado que, em primeira aproximação, as flutuações quânticas do campo podem contribuir para sua massa via o termo de autointeração de modo a tornar o campo mais ou menos estável, dependendo do acoplamento com a gravitação $\xi$ e de $\lambda$. O espaço-tempo de de Sitter é um caso especial dos espaçostempos de Friedmann-Lemaître-Robertson-Walker (FLRW),

$$
g_{a b}=-(d t)_{a}(d t)_{b}+a^{2}(t)\left[(d x)_{a}(d x)_{b}+(d y)_{a}(d y)_{b}+(d z)_{a}(d z)_{b}\right]
$$

\footnotetext{
${ }^{3}$ Para mais informação sobre a instabilidade de Schiff, Snyder e Weinberg e outras discutidas por Fulling na referência (70), remeto o leitor a parte 1 do Apêndice da referência (6) e as referências lá mencionadas.

${ }^{4}$ Agradeço ao Prof. A. A. Starobinsky por uma recente discussão a respeito desse assunto.
} 
onde o fator de expansão $a(t)$ é dado por

$$
a(t)=e^{H t}
$$

e $H$ é a constante de Hubble. A TQCEC sobre esse espaço-tempo foi bastante estudada a partir do final da década de 1970 por conta de suas simetrias e dos cenários inflacionários para o Universo primordial que foram propostos a partir de 1981. Nas referências $(75,76)$, em particular, são estudadas as propriedades dos estados de um campo escalar livre no espaço-tempo de de Sitter. Neles é mencionado que nesse espaço-tempo se $m^{2}+\xi R<0$ as flutuações crescem exponencialmente. De fato, os modos da Eq. (3.1.2) podem ser escolhidos como

$$
u_{\mathbf{k}}(t, \mathbf{x})=\frac{f_{\mathbf{k}}(t)}{a(t)} e^{i \mathbf{k} \cdot \mathbf{x}}
$$

onde $\mathbf{k} \in \mathbb{R}^{3}$ e $f_{\mathbf{k}}$ satisfaz

$$
\frac{d^{2}}{d \eta^{2}} f_{\mathbf{k}}+a^{2}\left[m^{2}+\frac{\|\mathbf{k}\|^{2}}{a^{2}}+12\left(\xi-\frac{1}{6}\right) H^{2}\right] f_{\mathbf{k}}=0
$$

sendo que $\eta=-H^{-1} e^{-H t}$ é o tempo conforme. Daí vê-se que, dependendo dos valores de $\xi$ e $m$, é possível que haja modos $u_{\mathbf{k}}$ que crescem exponencialmente. Em contextos cosmológicos há também estudos sobre as chamadas instabilidades taquiônicas - campos para os quais $m^{2}<0$ - em campos auto-interagentes e sua relação com fenômenos de criação espontânea de partículas (veja, e.g., a referência $(77)) .5$ É importante que se diga, contudo, que o Efeito de Despertar do Vácuo guarda diferenças em relação às instabilidades mencionadas, haja visto que aqui sempre tratei de um campo livre e cujo vácuo em Minkowski é naturalmente estável.

Ainda há, todavia, algumas perguntas referentes ao efeito que permanecem sem resposta. Talvez a maior delas seja a questão da retroação do campo, à qual chamei a atenção repetidas vezes ao longo do texto. Por um lado, não parece haver algoritmos numéricos para a construção do valor esperado do tensor energia-momentum do campo mesmo quando o espaço-tempo é dado a mão. Por outro, há o desafio numérico de se resol-

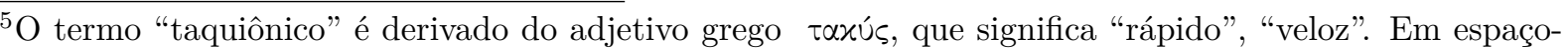
tempo plano, campos taquiônicos foram primeiramente estudados por G. Feinberg na referência (78) e sua quantização foi abordada com profundidade por B. Schröer na referência $(79)$.
} 
ver de forma autoconsistente a equação de Einstein semiclássica Eq. (3.4.1). De qualquer forma, é inegável que esse é um problema que precisa ser atacado, mesmo que inicialmente para o sistema puramente clássico, para que se tenha alguma pista de quais são as possíveis conseqüências da fase de instabilidades do campo para o sistema gravitacional. Outra questão de relevo é o possível despertar do vácuo em contextos cosmológicos. Já sabemos que para espaços-tempos de FLRW normalmente empregados para se descrever a evolução do Cosmos o efeito não é disparado. Portanto, a única possibilidade seria levar em conta as perturbações promovidas por uma estrutura cosmológica que, a rigor, não é homogênea e isotrópica. Por conta disso, esse é um problema que, mesmo sendo mais simples do que o problema da retroação, também requer uma abordagem numérica para a formação de estruturas no universo e, naturalmente, para resolver a equação para o campo escalar. Esse é um problema que temos estudado. Uma outra pergunta legítima é se o efeito é despertado para campos com spin $\frac{1}{2}$ ou 1. Para o campo de Dirac ainda é necessário investigação. Já no caso do campo eletromagnético sabemos que tanto no caso da estrela quanto no caso da casca esférica o efeito nunca é disparado para nenhum dos dois graus de liberdade de polarização. Para o caso da casca isso é particularmente surpreendente, uma vez que o efeito é disparado para o campo de KG com acoplamento conforme $\bigsqcup^{6}$ Essas negativas, contudo, não implicam que para espaços-tempos com menos simetrias o efeito não possa ser disparado para o vácuo do campo eletromagnético; esse é um problema que necessita de mais estudo.

\footnotetext{
${ }^{6}$ Pelo fato de compartilhar a invariância conforme com as equações de Maxwell, é normalmente considerado que o campo de KG com acoplamento conforme simule o campo do fóton.
} 


\section{Referências}

1 MiSnER, C. W.; THORNE, K. S.; WHEELER, J. A. Gravitation. San Francisco: W.H. Freeman and Company, 1973.

2 HAWKING, S. W.; ELLIS, G. F. R. The large scale structure of space-time. Cambridge: Cambridge University Press, 1973. (Cambridge Monographs on Mathematical Physics).

3 SACHS, R. K.; WU, H. General relativity for mathematicians. New York: SpringerVerlag, 1977. (Graduate Texts in Mathematics, 48).

4 WALD, R. M. General relativity. Chicago: The University of Chicago Press, 1984.

5 BIRRELL, N. D.; DAVIES, P. C. W. Quantum fields in curved space. Cambridge: Cambridge University Press, 1982. (Cambridge Monographs on Mathematical Physics).

6 FULling, S. A. Aspects of quantum field theory in curved space-time. Cambridge: Cambridge University Press, 1989. (London Mathematical Society Student Texts, 17).

7 WALD, R. M. Quantum field theory in curved spacetime and black hole thermodynamics. Chicago: The University of Chicago Press, 1994. (Chicago Lectures in Physics).

8 PARKER, L.; TOMS, D. Quantum field theory in curved spacetime: quantized fields and gravity. Cambridge: Cambridge University Press, 2009. (Cambridge Monographs on Mathematical Physics).

9 BÄR, C.; FREDENHAGEN, K. (Ed.). Quantum field theory on curved spacetimes: concepts and mathematical foundations. New York: Springer, 2009. (Lecture Notes in Physics, 786). 
10 BJORKEN, J. D.; DRELL, S. D. Relativistic quantum fields. New York: McGrawHill, 1964.

11 WEINBERG, S. The quantum theory of fields: foundations. Cambridge: Cambridge University Press, 1995. v. 1.

12 GOMES, M. O. C. Teoria quântica dos campos. São Paulo: Editora da Universidade de São Paulo, 2002. (Coleção Acadêmica, 39).

13 ZEE, A. Quantum field theory in a nutshell. Princeton: Princeton University Press, 2003.

14 WALD, R. M. The history and present status of quantum field theory in curved spacetime. Disponível em: <http://arxiv.org/abs/gr-qc/0608018>. Acesso em: 26 nov. 2011.

15 WALD, R. M. The formulation of quantum field theory in curved spacetime. Disponível em: <http://arxiv.org/abs/0907.0416>. Acesso em: 26 nov. 2011.

16 CASIMIR, H. G. B. On the attraction between two perfectly conducting plates. Proceedings of the Koninklijke Nederlandse Akademie van Wetenschappen, v. 51, p. 793 $795,1948$.

17 CHAN, H. B. et al. Quantum mechanical actuation of microelectromechanical systems by the Casimir force. Science, v. 291, n. 5510, p. 1941 - 1944, 2001.

18 LAMOREAUX, S. K. The Casimir force: background, experiments, and applications. Reports on Progress in Physics, v. 68, n. 1, p. 201 - 236, 2005.

19 PARKER, L. Particle creation in expanding universes. Physical Review Letters, v. 21, n. 8, p. $562-564,1968$.

20 HAWKING, S. W. Black hole explosions? Nature, v. 248, n. 5443, p. 30 - 31, 1974.

21 CASTIÑEIRAS, J. et al. Free massive particles with total energy $E<m c^{2}$ in curved spacetimes. Physical Review D, v. 65, n. 10, p. 104019, 2002.

22 BJORKEN, J. D.; DRELL, S. D. Relativistic quantum mechanics. New York: McGraw-Hill, 1964. 
23 SCHWINGER, J. On the gauge invariance and vacuum polarization. Physical Review v. 82, n. 5, p. $664-679,1951$.

24 LIMA, W. C. C.; VANZELLA, D. A. T. Gravity-induced vacuum dominance. Physical Review Letters, v. 104, n. 16, p. 161102, 2010.

25 LIMA, W. C. C.; MATSAS, G. E. A.; VANZELLA, D. A. T. Awaking the vacuum in relativistic stars. Physical Review Letters, v. 105, n. 15, p. 151102, 2010.

26 LAndulfo, A. G. S; LimA, W. C. C.; MATSAS, G. E. A.; VAnZELla, D. A. T. Burst of particles from the falling asleep of the vacuum (or tachyonic instability in relativistic stars). Disponível em: $<$ http://arxiv.org/abs/1204.3654 > . Acesso em: 10 maio 2012.

27 LIMA, W. C. C.; VANZELLA, D. A. T. Awaking the vacuum with spherical shells. Trabalho em andamento.

28 MALENFANT, J. Waking up the quantum vacuum. Physics. College Park. 20 abr. 2010. Disponível em: <http://physics.aps.org/synopsis-for/10.1103/ PhysRevLett.104.161102 >. Acesso em: 23 mar. 2011.

29 MONROE, D. Neutron star formation could awaken the vacuum. Physical Review Focus. College Park. 8 out. 2010. Disponível em:

$<$ http://focus.aps.org/story/v26/st14 > . Acesso em: 23 mar. 2011.

30 REED, M.; SIMON, B. Methods of modern mathematical physics: functional analysis. New York: Academic Press, 1972. v. 1.

31 KREYSZIG, E. Introductory functional analysis with applications. New York: John Wiley \& Sons, 1989.

32 BARATA, J. C. A. Curso de física matemática. Disponível em: <http: //denebola.if.usp.br/ jbarata/Notas_de_aula/notas_de_aula.html>. Acesso em: 15 ago. 2011.

33 BERNAL, A. N.; SÁNCHEZ, M. On smooth Cauchy hypersurfaces and Geroch's splitting theorem. Communications in Mathematical Physics, v. 243, n. 3, p. 461 - 470, 2003.

34 ASHTEKAR, A.; MAGNON, A. Quantum fields in curved space-times. Proceedings of the Royal Society A, v. 346, n. 1646, p. 375 - 394, 1975. 
35 WIGNER, E. Representations of the inhomogeneous Lorentz group. Annals of Mathematics, v. 40, n. 1, p. 149 - 204, 1939.

36 CHMIELOWSKI, P. States of scalar field on spacetimes with two isometries with timelike orbits. Classical and Quantum Gravity, v. 11, n. 1, p. 41 - 56, 1994.

37 DIMOCK, J. Algebras of local observables on a manifold. Communications in Mathematical Physics, v. 77, n. 3, p. 219 - 228, 1980.

38 HAAG, R. Local quantum physics: field, particles, algebras. Berlin: Springer-Verlag, 1996.

39 CALLAN, C. G.; COLEMAN, S.; JACKIW, R. A new improved energy-momentum tensor. Annals of Physics, v. 59, n. 1, p. 42 - 73, 1970.

40 FORGER, M.; RÖMER, H. Currents and the energy-momentum tensor in classical field theory: a fresh look at an old problem. Annals of Physics, v. 309, n. 2, p. 306 - 389, 1978.

41 KAY, B. S. Linear spin-zero quantum fields in external gravitational and scalar fields. Communications in Mathematical Physics, v. 62, n. 1, p. 55 - 70, 1978.

42 WALD, R. M. Existence of the $S$-matrix in quantum field theory in curved space-time. Annals of Physics, v. 118, n. 2, p. 490 - 510, 1979.

43 PARKER, L. Aspects of quantum field theory in curved space-time: effective action and energy-momentum tensor. In: LÉVY, M.; DESER, S. (Ed.) Recents developments in gravitation: Cargèse 1978. New York: Plenum Press, 1978, pg. 219. (NATO ASI Series. Series B: Physics, 44).

44 SIMON, B. The bound state of weakly coupled Schrödinger operators in one and two dimensions. Annals of Physics, v. 97, n. 2, p. 279 - 288, 1976.

45 LATTIMER, J. M.; PRAKASH, M. Neutron star structure and the equation of state. Astrophysical Journal, v. 550, n. 1, p. 426 - 442, 2001.

46 TOLMAN, R. C. Static solutions of Einstein's field equations for spheres of fluid. Physical Review, v. 55, n. 4, p. 364 - 373, 1939. 
47 POISSON, E. A relativist's toolkit: the mathematics of black-hole mechanics. Cambridge: Cambridge University Press, 2004.

48 ABRAMOWITZ, M. E.; STEGUN, I. A. (Ed.). Handbook of mathematical functions. New York: Dover, 1972.

49 IKEBE, T.; KATO, T. Uniqueness of the self-adjoint extension of singular elliptic differential operators. Archive for Rational Mechanics and Analysis, v. 9, n. 1, p. 77 - 92, 1962.

50 DONNELLY, H.; GAROFALO, N. Schrödinger operators on manifolds, essential self-adjointness, and absence of eigenvalues. Journal of Geometric Analysis, v. 7, n. 2, p. $241-257,1997$.

51 BARTON, G. Quantum mechanics of the inverted oscillator potential. Annals of Physics, v. 166, n. 2, p. 322 - 363, 1986.

52 TITCHMARSH, E. C. Eigenfunction expansions associated with second-order differential equations. Oxford: Oxford University Press, 1962. pt. 1.

53 GRADSHTEYN, I. S.; RYZHIK, I. M. (Ed.). Table of integrals, series and products. New York: Academic Press, 1965.

54 UNRUH, W. G. Particles and fields. In: AUDRESTSCH, J.; DE SABBATA, V. (Ed.). Quantum mechanics in curved space-time. New York: Plenum Press, 1990, pg. 89. (NATO ASI Series B, Physics, 230).

55 UNRUH, W. G. Notes on black hole evaporation. Physical Review D, v. 14, n. 4, p. $870-892,1976$.

56 CRISPINO, L. C. B.; HIGUSHI, A.; MATSAS, G. E. A. The Unruh effect and its applications. Reviews of Modern Physics, v. 80, n. 3, p. 787 - 838, 2008.

57 PARKER, L. Quantized fields and particle creation in expanding universes, I. Physical Review, v. 104, n. 16, p. 1057 - 1068, 1969.

58 HAWKING, S. W. Particle creation by black holes. Communications in Mathematical Physics, v. 43, n. 3, p. 199 - 220, 1975. 
59 DEWITT, B. S. Quantum gravity: the new synthesis. In: HAWKING, S. W.; ISRAEL, W. (Ed.). General relativity: an Einstein centenary survey. Cambridge: Cambridge University Press, 1979, cap. 14.

60 FUlling, S. A.; SWEENY, M.; WALD, R. M. Singularity structure of the two-point function quantum field theory in curved spacetime. Communications in Mathematical Physics, v. 63, n. 3, p. 257 - 264, 1978.

61 FULLING, S. A.; NARCOWICH, F. J.; WALD, R. M. Singularity structure of the two-point function in quantum field theory in curved spacetime, II. Annals of Physics, v. 136, n. 2, p. $243-272,1981$.

62 RADZIKOWSKI, M. J. A local-to-global singularity theorem for quantum field theory on curved space-time. Communications in Mathematical Physics, v. 180, n. 1, p. $1-22,1996$.

63 PANI, P. et al. Vacuum revealed: the final state of vacuum instabilities in compact stars. Physical Review D, v. 83, n. 8, p. 081501, 2011.

64 DAMOUR, T.; ESPOSITO-FARÈSE, G. Nonpertubative strong-field effects in tensor-scalar theories of gravitation. Physical Review Letters, v. 70, n. 15, p. 2220 - 2223, 1993.

65 DAMOUR, T.; ESPOSITO-FARÈSE, G. Tensor-scalar gravity and binary-pulsar experiments. Physical Review D, v. 54, n. 2, p. 1474 - 1491, 1996.

66 DE FELICE, A.; TSUJIKAWA, S. $f(R)$ theories. Living Reviews in Relativity, v. 13, n. 3, 2010. Disponível em: <http://relativity.livingreviews.org/Articles/ 1rr-2010-3/>. Acesso em: 10 maio 2012.

67 HARADA, T. Stability analysis of spherically symmetric star in scalar-tensor theories of gravity. Progress of Theoretical Physics, v. 98, n. 2, p. 359 - 379, 1997.

68 SCHIFF, L. I.; SNYDER, H.; WEINBERG, J. On the existence of stationary states of the mesotron field. Physical Review, v. 57, n. 4, p. 315 - 318, 1940.

69 SCHRÖER, B.; SWIECA, J. A. Indefinite metric and stationary external interactions of quantized fields. Physical Review D, v. 2, n. 12, p. 2938 - 2944, 1970.

70 FULLING, S. A. Varieties of instability of a boson field in an external potential. Physical Review D, v. 14, n. 8, p. 1939 - 1943, 1976. 
71 FORD, L. H.; TOMS, D. J. Dynamical symmetry breaking due to radiative corretions in cosmology. Physical Review D, v. 25, n. 6, p. 1510, 1982.

72 VILENKIN, A.; FORD, L. H. Gravitational effects upon cosmologycal phase transitions. Physical Review D, v. 26, n. 6, p. 1231, 1982.

73 LINDE, A. D. Scalar field fluctuations in the expanding universe and the new inflationary universe scenario. Physics Letters, v. 116B, n. 5, p. 335, 1982.

74 STAROBINSKY, A. A. Dynamics of phase transition in the new inflationary universe scenario and generation of perturbations. Physics Letters, v. 117B, n. 3, p. 175, 1982.

75 ANDERSON, P. R. et al. Attractor states and infrared scaling in de Sitter space. Physical Review D, v. 62, n. 12, p. 124019, 2000.

76 ANDERSON, P. R. et al. Attractor states and quantum instabilities in de Sitter space. International Journal of Theoretical Physics, v. 40, n. 12, p. 2217, 2001.

77 FELDER, G.; KOFMAN, L.; LINDE, A. Tachyonic instability and dynamics of spontaneous symmetry breaking Physical Review D, v. 64, n. 12, p. 123517, 2001.

78 FEINBERG, G. Possibility of faster-than-light particles. Physical Review, v. 159, n. 5, p. 1089 - 1105, 1967.

79 SCHRÖER, B. Quantization of $m^{2}<0$ field equations. Physical Review D, v. 3, n. 8, p. 1764 - 1770, 1971. 


\section{Apêndice A}

\section{Prova da desigualdade Eq. (7.1.6)}

\section{para a Eq. (7.1.5)}

Suponha um espaço-tempo $(\mathcal{M}, g)$ estático e considere sobre ele um campo escalar de KG satisfazendo a Eq. (3.1.2). Neste apêndice desejo provar que o produto interno $\langle\cdot, \cdot\rangle_{|\bar{\Delta}|}$ : $\mathscr{S}^{\mathbb{C}} \times \mathscr{S}^{\mathbb{C}} \rightarrow \mathbb{C}$ definido por

$$
\left\langle\psi_{1}, \psi_{2}\right\rangle_{|\bar{\Delta}|}:=\frac{1}{2} \int_{\Sigma_{t}} \frac{d \Sigma}{\|\varkappa\|}\left(\bar{p}_{1} p_{2}+\bar{\phi}_{1}|\bar{\Delta}| \phi_{2}\right)
$$

satisfaz, para algum $C>0$,

$$
C^{2}\left\langle\psi_{1}, \psi_{1}\right\rangle_{|\bar{\Delta}|}\left\langle\psi_{2}, \psi_{2}\right\rangle_{|\bar{\Delta}|} \geq \frac{1}{4}\left|\Omega\left(\psi_{1}, \psi_{2}\right)\right|^{2}
$$

Assim como antes, $\Delta$ denota o operador diferencial

$$
-\|\varkappa\| D^{a}\left(\|\varkappa\| D_{a} \cdot\right)+\|\varkappa\|^{2}\left(m^{2}+\xi R\right) \cdot,
$$

enquanto que a forma simplética $\Omega$, definida pela Eq. (3.1.5), pode ser escrita como

$$
\Omega\left(\bar{\psi}_{1}, \psi_{2}\right)=\int_{\Sigma_{t}} \frac{d \Sigma}{\|\varkappa\|}\left(\bar{p}_{1} \phi_{2}-p_{2} \bar{\phi}_{1}\right)
$$

onde $\psi=(\phi, \pi) \in \mathscr{S}_{\mu}^{\mathbb{C}}, \pi=\sqrt{h} p$ e $p=\varkappa^{a} \nabla_{a} \phi$.

Suponha que para a superfície de Cauchy $\Sigma_{t}$ existam as constantes $\varepsilon_{1}, \varepsilon_{2}>0$ tais que 
a norma do campo de Killing tipo tempo satisfaz

$$
\varepsilon_{1} \leq\|\varkappa\| \leq \varepsilon_{2}
$$

O primeiro passo da prova consiste na seguinte desigualdade:

$$
\begin{aligned}
\left|\Omega\left(\bar{\psi}_{1}, \psi_{2}\right)\right|^{2}= & \left|\int_{\Sigma_{t}} \frac{d \Sigma}{\|\varkappa\|}\left(\bar{p}_{1} \phi_{2}-p_{2} \bar{\phi}_{1}\right)\right|^{2} \\
\leq & \frac{1}{\varepsilon_{1}^{2}}\left[\int_{\Sigma_{t}} d \Sigma\left(\left|p_{1}\right|\left|\phi_{2}\right|+\left|p_{2}\right|\left|\bar{\phi}_{1}\right|\right)\right]^{2} \\
\leq & \frac{1}{\varepsilon_{1}^{2}}\left[\left(\int_{\Sigma_{t}} d \Sigma\left|p_{1}\right|^{2}\right)^{1 / 2}\left(\int_{\Sigma_{t}} d \Sigma\left|\phi_{2}\right|^{2}\right)^{1 / 2}\right. \\
& \left.+\left(\int_{\Sigma_{t}} d \Sigma\left|p_{2}\right|^{2}\right)^{1 / 2}\left(\int_{\Sigma_{t}} d \Sigma\left|\phi_{1}\right|^{2}\right)^{1 / 2}\right]^{2} \\
\leq & \frac{1}{\varepsilon_{1}^{2}} \int_{\Sigma_{t}} d \Sigma\left(\left|p_{1}\right|^{2}+\left|\phi_{1}\right|^{2}\right) \int_{\Sigma_{t}} d \Sigma\left(\left|p_{2}\right|^{2}+\left|\phi_{2}\right|^{2}\right)
\end{aligned}
$$

Na segunda desigualdade foi usado que, pela desigualdade de Cauchy-Schwarz,

$$
\int_{\Sigma_{t}} d \Sigma\left|p_{1}\right|\left|\phi_{2}\right| \leq\left(\int_{\Sigma_{t}} d \Sigma\left|p_{1}\right|^{2}\right)^{1 / 2}\left(\int_{\Sigma_{t}} d \Sigma\left|\phi_{2}\right|^{2}\right)^{1 / 2}
$$

enquanto que na terceira foi usado que

$$
|a||b|+|c||d| \leq \sqrt{|a|^{2}+|d|^{2}} \sqrt{|b|^{2}+|c|^{2}}
$$

o que também segue da desigualdade de Cauchy-Schwarz.

Por outro lado, se para todo $\lambda \in \sigma(\bar{\Delta})$, o espectro de $\bar{\Delta}$, existir uma constante $M>0$ tal que $|\lambda|>M$, então

$$
\begin{aligned}
\left\langle\psi_{1}, \psi_{1}\right\rangle_{|\bar{\Delta}|} & \geq \frac{1}{2 \varepsilon_{2}} \int_{\Sigma_{t}} d \Sigma\left(\left|p_{1}\right|^{2}+M\left|\phi_{1}\right|^{2}\right) \\
& \geq \frac{1}{2 \varepsilon_{2}} \min (1, M) \int_{\Sigma_{t}} d \Sigma\left(\left|p_{1}\right|^{2}+\left|\phi_{1}\right|^{2}\right) .
\end{aligned}
$$

Finalmente, combinando as desigualdades Eq. A.6 e Eq. A.9 vem que

$$
\left|\Omega\left(\bar{\psi}_{1}, \psi_{2}\right)\right|^{2} \leq \frac{4 \varepsilon_{2}^{2}}{\varepsilon_{1}^{2}} \frac{1}{\min (1, M)^{2}}\left\langle\psi_{1}, \psi_{1}\right\rangle_{|\bar{\Delta}|}\left\langle\psi_{2}, \psi_{2}\right\rangle_{|\bar{\Delta}|},
$$

e assim chega-se à desigualdade desejada.1 1

\footnotetext{
${ }^{1}$ Agradeço ao Prof. J. C. A. Barata por apontar um erro de minha parte na prova original da desigualdade Eq. A.6 e por me apresentar os passos que agora levam àquele resultado.
} 